\title{
Organocatalytic Approach to Photochemical Lignin Fragmentation
}

Cheng Yang, ${ }^{\dagger, \nabla}$ Markus D. Kärkäs, ${ }^{\dagger,}, \nabla$ Gabriel Magallanes, ${ }^{\dagger}, \nabla$ Kimberly Chan ${ }^{\dagger}$ and Corey R. J. Stephenson*,†

$\nabla$ These authors contributed equally.

${ }^{\dagger}$ Department of Chemistry, University of Michigan, 930 North University Avenue, Ann Arbor, Michigan 48109, United States.

* Department of Chemistry, KTH Royal Institute of Technology, SE-100 44 Stockholm, Sweden.

*Corresponding author, Email: crjsteph@umich.edu. 


\section{Contents}

1. General information

2. Synthesis and characterization of $N$-phenylphenothiazine (PhPTH)

3. Optimization of reaction conditions for organocatalytic photochemical fragmentation

4. Organocatalytic photochemical reductive $\mathrm{C}-\mathrm{O}$ bond cleavage of aromatic ketones

5. Organocatalytic photochemical reductive $\mathrm{C}-\mathrm{O}$ bond cleavage of $1 \mathrm{a}$ and $1 \mathrm{~b}$ in continuous flow

6. One-pot, two-step approaches to lignin fragmentation

7. Characterization of products $2 \mathrm{a}-2 \mathrm{e}$ and $3 \mathrm{a}-3 \mathrm{~b}$

8. Characterization of substrates $1 \mathrm{a}-1 \mathrm{i}, 1 \mathrm{ared}-1 \mathrm{~d}$ red, $1 \mathrm{f}_{\text {red, }}$ and $1 \mathrm{~h}$ red

9. Photon flux determination

10. Proposed mechanism of PhPTH-catalyzed photochemical reductive cleavage

11. References

12. NMR spectra 


\section{General information}

Commercially available chemicals were used as received. The substrates used for the one-pot oxidation/reductive C-O bond cleavage reactions were prepared according to reported procedures. ${ }^{[\mathrm{Si}, \mathrm{Sii}]}$ Merck silica gel 60 F254 TLC plates was used for thin-layer chromatography (TLC) analysis of reaction mixtures, visualized by a dual short wave/long wave UV lamp and stained using $p$-anisaldehyde. Purification by column chromatography was performed using 230 - 400 mesh silica gel.

Photochemical reactions were performed in a borosilicate vial irradiated by either violet LED strips or Kessil LEDs without the use of any filters. The detailed information is below.

1) The violet LED strips (double density 5050 flexible LED strips) are purchased from Creative Lighting Solutions. The maximum light intensity centered at $400-410 \mathrm{~nm}$.

https:/www.creativelightings.com/Purple-UV-LED-Flex-Strips-12vdc-WP-300-Meter-p/cl-frs5050wpdd$\underline{5 \mathrm{~m}-12 \mathrm{v}-\mathrm{uv} \cdot \mathrm{htm}}$

2) The Kessil LEDs (PR160-390 nm) are purchased from Kessil. The maximum light intensity centered at $390 \mathrm{~nm}$.

https://www.kessil.com/photoreaction/PR160L.php

3) The 2 dram clear borosilicate vial is purchased from Fisher.

https://www.fishersci.com/shop/products/fisherbrand-class-b-clear-glass-threaded-vials-

$\underline{\text { 37/0333922d?searchHijack=true\&searchTerm=0333922D\&searchType=RAPID\&matchedCatNo=03339 }}$

$\underline{22 \mathrm{D}}$

Bulk electrolysis was carried out using $\mu$ Stat 4000 Multi-Channel Potentiostat/Galvanostat (from Metrohm USA). UV-vis spectra were recorded on a Shimadzu UV-1601 UV-VIS spectrometer equipped with a Peltier temperature controller. The reaction setup is as the same as that reported in ref. S6.

Nuclear magnetic resonance (NMR) spectra were recorded using an internal deuterium lock on Varian MR400, MR500 and Varian Inova 500 spectrometers at the Department of Chemistry instrumentation facility, University of Michigan, Ann Arbor, MI. Chemical shifts $(\delta)$ are reported in parts per million (ppm), and are quoted to two decimal places and a single decimal place for ${ }^{1} \mathrm{H}$ and ${ }^{13} \mathrm{C}$ NMR, respectively, with coupling constants $(J)$ expressed in hertz $(\mathrm{Hz})$ to the nearest $0.1 \mathrm{~Hz}$. The chemical shifts are reported relative to the residual protio or deuterated solvent resonance $\left(\mathrm{CDCl}_{3}: \delta \mathrm{H}=7.26 \mathrm{ppm}\right.$ and $\delta \mathrm{C}=77.16 \mathrm{ppm} ; \mathrm{CD}_{3} \mathrm{CN}: \delta \mathrm{H}=1.94 \mathrm{ppm}$ and $\delta \mathrm{C}=118.26 \mathrm{ppm}$; acetone$d_{6}: \delta \mathrm{H}=2.05 \mathrm{ppm}$ and $\delta \mathrm{C}=29.84 \mathrm{ppm}$ ). Multiplicities are reported using the following abbreviations: $\mathrm{s}=$ singlet, $\mathrm{d}=$ doublet, $\mathrm{t}=$ triplet, $\mathrm{q}=$ quartet, quint $=$ quintet, $\mathrm{m}=$ multiplet, $\mathrm{br} \mathrm{s}=$ broad singlet, $\mathrm{dd}=$ doublet of doublet, $\mathrm{dt}$ 
$=$ doublet of triplet, etc. High-resolution mass spectra (HRMS) were obtained on a Micromass AutoSpec Ultima Magnetic Sector mass spectrometer using electrospray ionization (ESI), positive ion mode on an Agilent Q-TOF (model G6520B) HPLC-MS system, at the Department of Chemistry instrumentation facility, University of Michigan, Ann Arbor, MI. 


\section{Synthesis and characterization of $N$-phenylphenothiazine (PhPTH)}

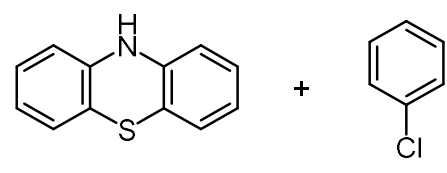

RuPhos precat. (2 mol\%)

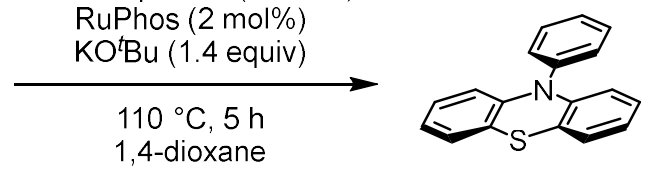

$N$-phenylphenothiazine was prepared according to the reported procedure. ${ }^{[\mathrm{S} 3]}$ To a vial equipped with a stir bar was added phenothiazine (199 mg, $1.0 \mathrm{mmol}$ ), RuPhos precatalyst (16.3 mg, $0.020 \mathrm{mmol}, 2.0 \mathrm{~mol} \%$, Sigma-Aldrich catalogue number: 707589), RuPhos (9.3 mg, $0.020 \mathrm{mmol}, 2.0 \mathrm{~mol} \%$, Sigma-Aldrich catalogue number: 663131) and $\mathrm{KO}^{t} \mathrm{Bu}(157 \mathrm{mg}, 1.40 \mathrm{mmol})$. The vial was evacuated and backfilled with nitrogen $(3 \times)$ before adding anhydrous 1,4-dioxane $(1.0 \mathrm{~mL})$ and anhydrous chlorobenzene $(158 \mathrm{mg}, 143 \mu \mathrm{L}, 1.40 \mathrm{mmol})$. The reaction was heated at 110 ${ }^{\circ} \mathrm{C}$ (heating mantle) for $5 \mathrm{~h}$, and then cooled to room temperature, diluted with $\mathrm{CH}_{2} \mathrm{Cl}_{2}$ and water. The phases were separated, and the aqueous layer was additionally extracted with $\mathrm{CH}_{2} \mathrm{Cl}_{2}(3 \times)$. The combined organic phases were washed with brine, dried over $\mathrm{Na}_{2} \mathrm{SO}_{4}$, and evaporated under reduced pressure. The crude product was purified by silica gel chromatography (hexanes/EtOAc 95:5) to afford the product as a white crystalline solid (163 mg, 59\%).

\section{Characterization of $N$-phenylphenothiazine}

$\mathbf{R}_{\mathbf{f}}=0.56$ (hexanes/EtOAc 95:5); ${ }^{1} \mathbf{H}$ NMR (500 MHz, $\left.\mathbf{C D C l}_{3}\right) \delta 7.60(\mathrm{t}, J=7.6 \mathrm{~Hz}, 2 \mathrm{H}), 7.48(\mathrm{t}, J=7.6 \mathrm{~Hz}, 1 \mathrm{H})$, $7.39(\mathrm{~d}, J=7.6 \mathrm{~Hz}, 2 \mathrm{H}), 7.02(\mathrm{dd}, J=7.4,1.6 \mathrm{~Hz}, 2 \mathrm{H}), 6.84(\mathrm{td}, J=7.6,1.6 \mathrm{~Hz}, 2 \mathrm{H}), 6.80$ (td, $J=7.3,1.1 \mathrm{~Hz}, 2 \mathrm{H})$, $6.20(\mathrm{dd}, J=8.0,1.1 \mathrm{~Hz}, 2 \mathrm{H}) ;{ }^{13} \mathbf{C}$ NMR (126 MHz, $\left.\mathbf{C D C l}_{3}\right) \delta 144.4$ (2C), 141.1, 131.0 (2C), 130.9 (2C), 128.3 , 127.0 (2C), 126.8 (2C), 122.6 (2C), 120.3 (2C), 116.2 (2C); HRMS (ESI) $m / z:[\mathrm{M}+\mathrm{H}]^{+}$Calcd for $\mathrm{C}_{18} \mathrm{H}_{14} \mathrm{NS}$ 276.0841 , Found 276.0835 .

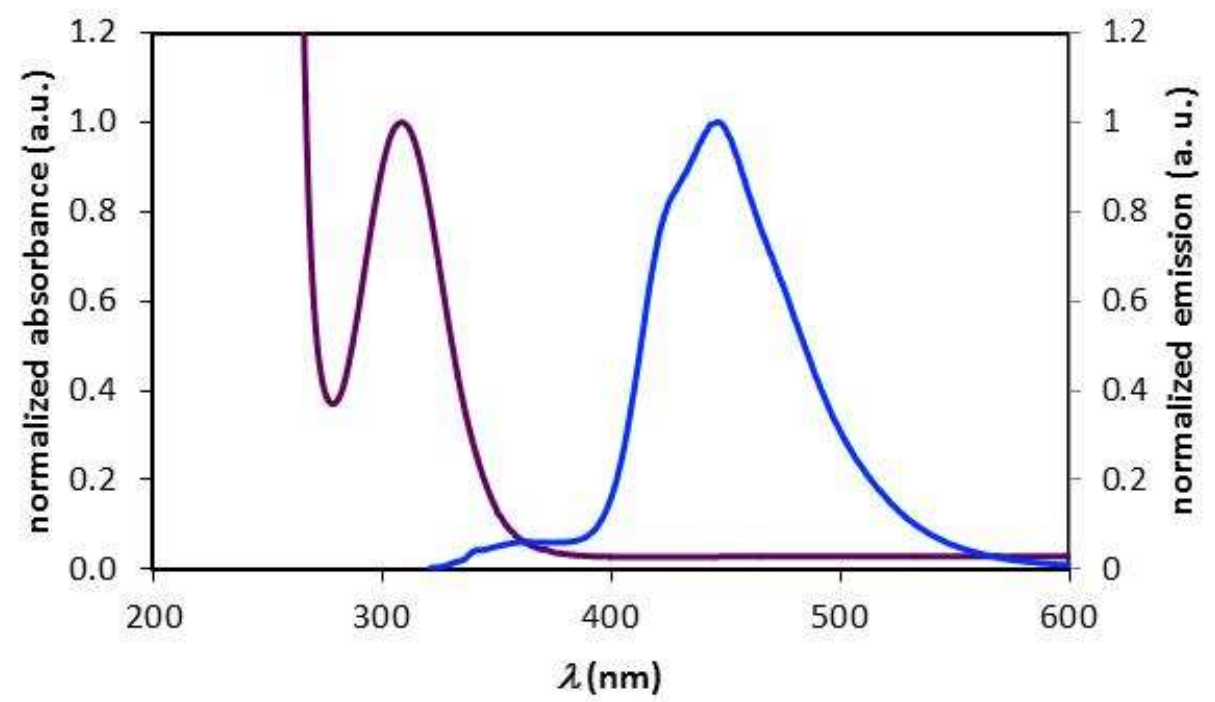

Figure S1. Absorption (purple) and emission (blue) spectra of PhPTH in acetone solutions. 


\section{Optimization of reaction conditions for organocatalytic photochemical fragmentation}

Table S1. Optimization of the photochemical reductive C-O bond cleavage. ${ }^{[a]}$
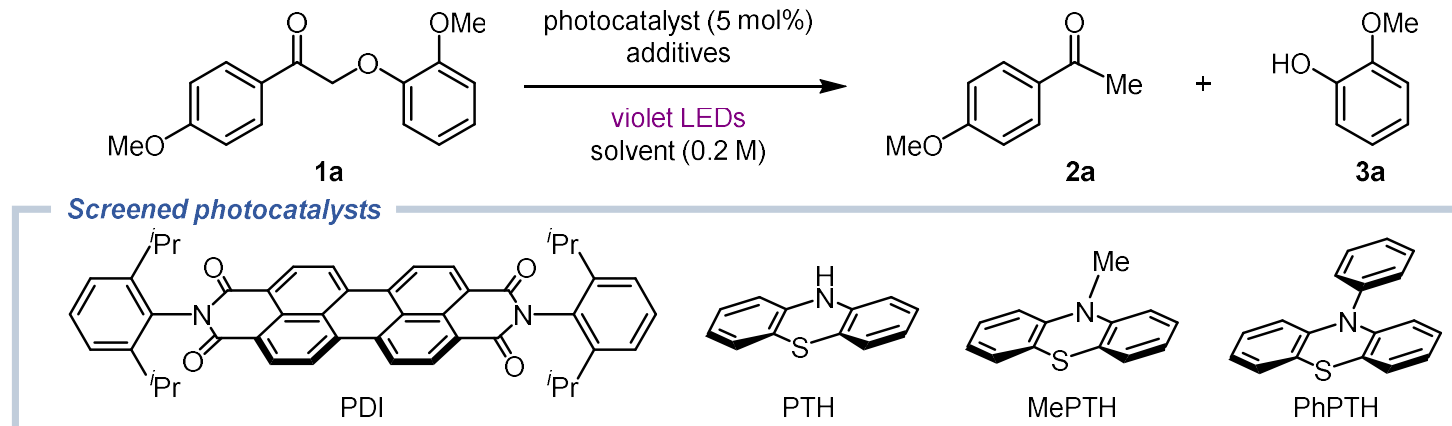

\begin{tabular}{|c|c|c|c|c|c|c|}
\hline entry & photocatalyst & solvent & amine & $\begin{array}{l}\mathrm{HCO}_{2} \mathrm{H} \\
\text { (equiv.) }\end{array}$ & time/h & $\begin{array}{r}{ }^{1} \mathrm{H} \text { NMR } \\
\text { yield/\% }{ }^{[\mathrm{b}]}\end{array}$ \\
\hline 1 [c] & PDI & $\mathrm{MeCN}$ & $\mathrm{Pr}_{2} \mathrm{NEt}$ & 0.1 & 24 & $<5$ \\
\hline $2^{[c]}$ & PDI & acetone & ${ }^{2} \mathrm{Pr}_{2} \mathrm{NEt}$ & 0.1 & 24 & $<5$ \\
\hline $3^{[c]}$ & PDI & acetone & ${ }^{i} \mathrm{Pr}_{2} \mathrm{NEt}$ (8 equiv.) & - & 24 & $<5$ \\
\hline 4 & PTH & acetone & ${ }^{i} \mathrm{Pr}_{2} \mathrm{NEt}$ & 1.0 & 24 & 8 \\
\hline 5 & МePTH & acetone & $\mathrm{Pr}_{2} \mathrm{NEt}$ & 1.0 & 24 & 10 \\
\hline 6 & PhPTH & acetone $(0.2 \mathrm{M})$ & $\mathrm{Pr}_{2} \mathrm{NEt}$ & 1.0 & 24 & 30 \\
\hline 7 & PhPTH & $\mathrm{MeCN}$ & ${ }^{i} \mathrm{Pr}_{2} \mathrm{NEt}$ & 1.0 & 24 & 13 \\
\hline 8 & PhPTH & THF & $\mathrm{Pr}_{2} \mathrm{NEt}$ & 1.0 & 24 & 10 \\
\hline 9 & PhPTH & $\mathrm{MeNO}_{2}$ & ${ }^{i} \mathrm{Pr}_{2} \mathrm{NEt}$ & 1.0 & 24 & $<5$ \\
\hline 10 & PhPTH & DCE & $\mathrm{Pr}_{2} \mathrm{NEt}$ & 1.0 & 24 & 20 \\
\hline 11 & PhPTH & $\mathrm{MeOH}$ & ${ }^{i} \mathrm{Pr}_{2} \mathrm{NEt}$ & 1.0 & 24 & 9 \\
\hline 12 & PhPTH & acetone & $\mathrm{Et}_{3} \mathrm{~N}$ & 1.0 & 24 & $<5$ \\
\hline 13 & PhPTH & acetone & $\mathrm{Bu}_{3} \mathrm{~N}$ & 1.0 & 24 & 16 \\
\hline 14 & PhPTH & acetone (0.4 M) & ${ }^{2} \mathrm{Pr}_{2} \mathrm{NEt}$ & 1.0 & 24 & 32 \\
\hline 15 & PhPTH & acetone (0.1 M) & ${ }^{2} \mathrm{Pr}_{2} \mathrm{NEt}$ & 1.0 & 24 & 42 \\
\hline 16 & PhPTH & acetone $(0.05 \mathrm{M})$ & ${ }^{2} \mathrm{Pr}_{2} \mathrm{NEt}$ & 1.0 & 24 & 48 \\
\hline 17 & PhPTH & acetone $(0.05 \mathrm{M})$ & ${ }^{i} \mathrm{Pr}_{2} \mathrm{NEt}$ & 1.0 & 96 & $>95$ \\
\hline 18 & PhPTH & acetone $(0.05 \mathrm{M})$ & ${ }^{1} \mathrm{Pr}_{2} \mathrm{NEt}$ & - & 96 & 78 \\
\hline 19 & - & acetone $(0.05 \mathrm{M})$ & ${ }^{2} \mathrm{Pr}_{2} \mathrm{NEt}$ & 1.0 & 96 & 15 \\
\hline 20 & PhPTH & acetone $(0.05 \mathrm{M})$ & - & 1.0 & 96 & $<5$ \\
\hline $21^{[\mathrm{d}]}$ & PhPTH & acetone $(0.05 \mathrm{M})$ & ${ }^{2} \mathrm{Pr}_{2} \mathrm{NEt}$ & 1.0 & 96 & $<5$ \\
\hline
\end{tabular}

[a] Reaction conditions: $1 \mathrm{a}(0.1 \mathrm{mmol})$, photocatalyst $(5 \mathrm{~mol} \%)$, amine (2 equiv.) and formic acid (1 equiv.) were dissolved in the selected solvent $(0.5 \mathrm{~mL}, 0.2 \mathrm{M})$ unless otherwise noted. ${ }^{[b]}{ }^{1} \mathrm{H}$ NMR yields of $2 \mathrm{a}$ were determined by ${ }^{1} \mathrm{H}$ NMR using 1,3,5-trimethoxybenzene as internal standard. ${ }^{[c]}$ Reaction was irradiated with blue LEDs. [d] Reaction was carried out in the dark.

\section{General procedures}

Substrate 1a $(0.1 \mathrm{mmol})$ were dissolved in acetone $(0.5 \mathrm{~mL})$, followed by adding photocatalyst $(0.005 \mathrm{mmol}, 5$ mol\%) and additives. The resulting solution was sparged with nitrogen for 5 min before irradiated with LEDs at ambient temperature. The LEDs were placed surrounding the reactor (clear borosilicate glass vial) approximately 3 $\mathrm{cm}$ away from it. No filter was used. A fan was placed above the reaction to control the temperature. The After irradiation, the reaction mixture was concentrated under reduced pressure and dried in vacuo. The yields were determined by ${ }^{1} \mathrm{H}$ NMR using 1,3,5-trimethoxybenzene as internal standard. 


\section{Organocatalytic photochemical reductive $\mathrm{C}-\mathrm{O}$ bond cleavage of aromatic ketones}

\section{General procedures}

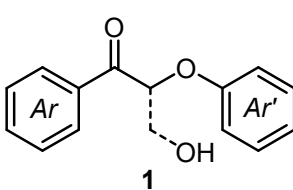

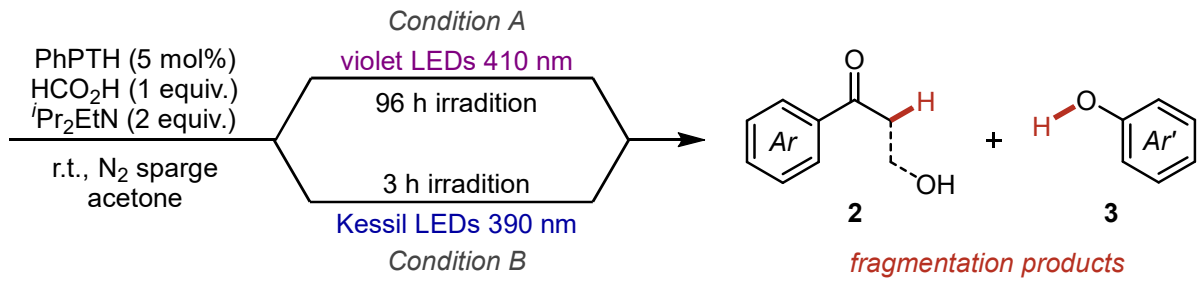

Condition A. Oxidized lignin model substrates $1(0.25 \mathrm{mmol})$ were dissolved in acetone $(5 \mathrm{~mL})$, followed by adding the PhPTH photocatalyst $(0.0125 \mathrm{mmol}, 5 \mathrm{~mol} \%)$, formic acid $(0.25 \mathrm{mmol}, 1$ equiv.) and diisopropylethylamine ( $0.5 \mathrm{mmol}, 2$ equiv.). The resulting solution was sparged with nitrogen for $5 \mathrm{~min}$ before irradiated with violet LED strips at ambient temperature. The violet LED strips (LED array $3 \times 15)$ were placed surrounding the reactor $(2$ dram clear borosilicate glass vial) approximately $3 \mathrm{~cm}$ away from it (Figure S2, left). No filter was used. A fan was placed above the reaction to control the temperature. The After $96 \mathrm{~h}$ of irradiation, the reaction mixture was concentrated under reduced pressure and dried in vacuo. The crude residue was purified by column chromatography to afford the desired products $\mathbf{2}$ and $\mathbf{3}$.

Condition B. Oxidized lignin model substrates $1(0.25 \mathrm{mmol})$ were dissolved in acetone $(5 \mathrm{~mL})$, followed by adding the $\mathrm{PhPTH}$ photocatalyst $(0.0125 \mathrm{mmol}, 5 \mathrm{~mol} \%)$, formic acid $(0.25 \mathrm{mmol}, 1$ equiv.) and diisopropylethylamine ( $0.5 \mathrm{mmol}, 2$ equiv.). The resulting solution was sparged with nitrogen for 5 min before irradiated with Kessil PR160-390 nm at ambient temperature. One Kessil LED (PR160-390 nm) was placed next to the reactor (2 dram clear borosilicate glass vial) approximately $3 \mathrm{~cm}$ away from it (Figure S2, right). No filter was used. A fan was placed above the reaction to control the temperature. After $3 \mathrm{~h}$ of irradiation, the reaction mixture was concentrated under reduced pressure and dried in vacuo. The crude residue was purified by column chromatography to afford the desired products $\mathbf{2}$ and $\mathbf{3}$.
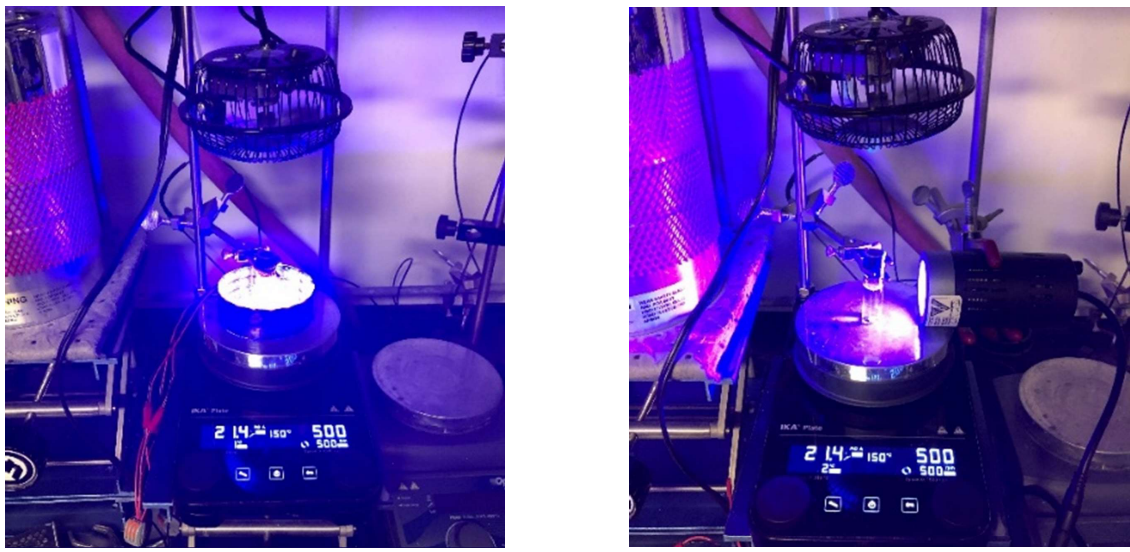

Figure S2. Reaction setup of condition A (left) and condition B (right). 


\section{Reaction results}<smiles>COc1ccc(C(=O)COc2ccccc2OC)cc1</smiles>

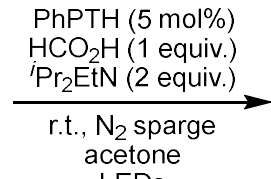<smiles>COc1ccc(C(C)=O)cc1</smiles><smiles>COc1ccccc1O</smiles>

Condition A: violet LED strips, 96 h, 2a (35 mg, 94\% yield) and 3a (29 mg, 92\% yield).

Condition B: Kessil PR160-390 nm, 3h, 2a (30 mg, 79\% yield) and 3a (26 mg, 85\% yield).<smiles>COc1ccc(C(=O)C(CO)Oc2ccccc2OC)cc1</smiles>

$1 \mathrm{~b}$

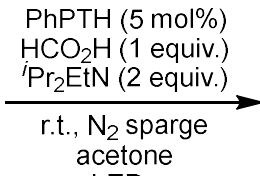

LEDs

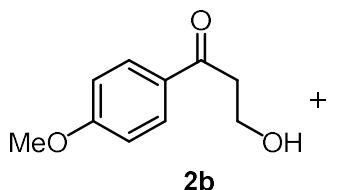

2b<smiles>COc1ccccc1O</smiles>

$3 a$

Condition A: violet LED strips, 96 h, $2 \mathbf{b}$ (42 mg, 93\% yield) and 3a (28 mg, 90\% yield).

Condition B: Kessil PR160-390 nm, 3h, 2b (41 mg, 91\% yield) and 3a (24 mg, 76\% yield).<smiles>COc1ccc(C(=O)COc2ccccc2OC)cc1OC</smiles>

$1 c$

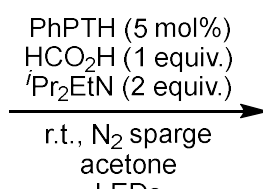

LEDs<smiles>COc1ccc(C(C)=O)cc1OC</smiles>

2c<smiles>COc1ccccc1O</smiles>

$3 a$

Condition A: violet LED strips, 96 h, 2c (41 mg, 92\% yield) and 3a (28 mg, 89\% yield).

Condition B: Kessil PR160-390 nm, 3h, 2c (30 mg, 67\% yield) and 3a (29 mg, 95\% yield).<smiles>COc1ccc(C(=O)C(CO)Oc2ccccc2OC)cc1OC</smiles>

$1 d$

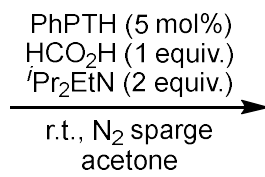

LEDs

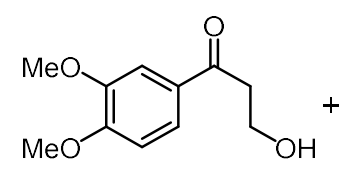

$2 d$<smiles>COc1ccccc1O</smiles>

$3 a$

Condition A: violet LED strips, 96 h, 2c (48 mg, 91\% yield) and 3a (29 mg, 93\% yield).

Condition B: Kessil PR160-390 nm, 3h, 2c (45 mg, 86\% yield) and 3a (25 mg, 81\% yield). 

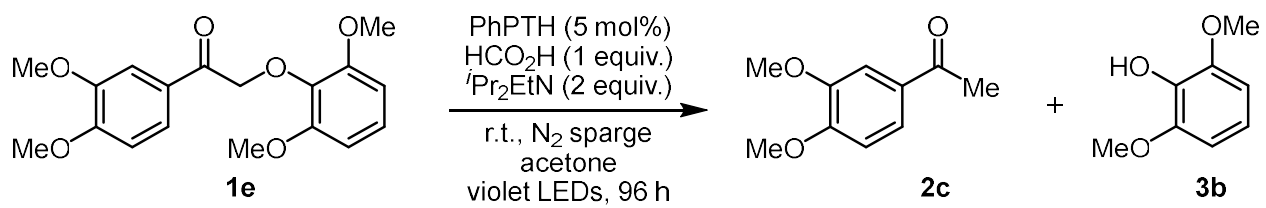

Condition A: violet LED strips, 96 h, 2c (42 mg, 94\% yield) and 3a (36 mg, 92\% yield).
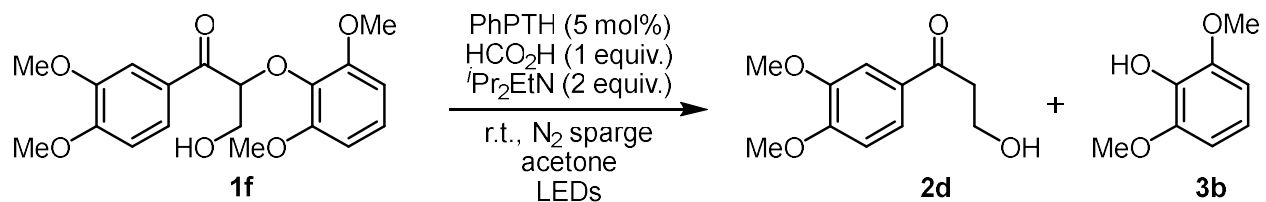

Condition A: violet LED strips, 96 h, 2c (47 mg, 90\% yield) and 3a (36 mg, 93\% yield).

Condition B: Kessil PR160-390 nm, 3h, 2c (36 mg, 69\% yield) and 3a (19 mg, 50\% yield).
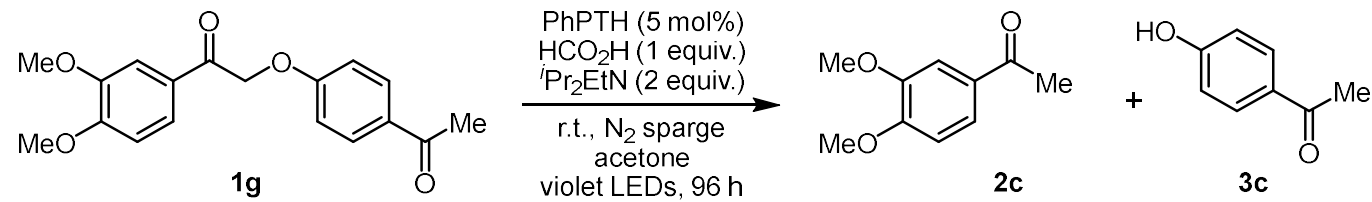

Condition A: violet LED strips, 96 h, 2c (43 mg, 95\% yield) and 3a (32 mg, 94\% yield).
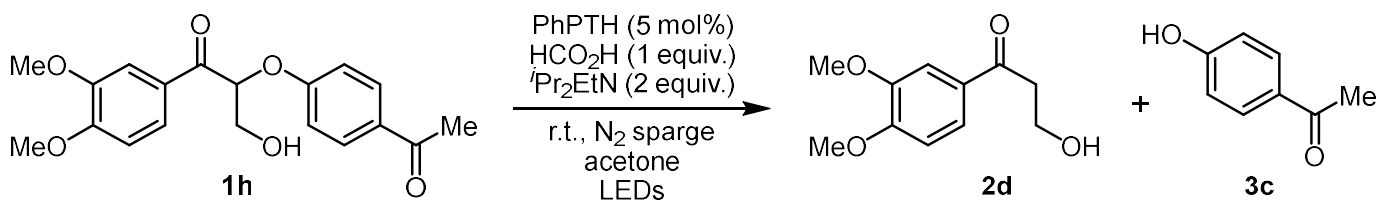

Condition A: violet LED strips, 96 h, 2c (47 mg, 90\% yield) and 3a (31 mg, 93\% yield).

Condition B: Kessil PR160-390 nm, 3h, 2c (35 mg, 66\% yield) and 3a (32 mg, 95\% yield).<smiles>COc1ccccc1OC(CO)C(=O)c1cc(OC)c(OC)c(OC)c1</smiles>

$1 \mathrm{i}$

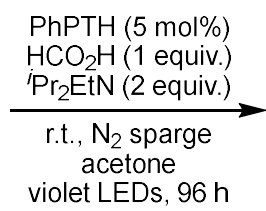

violet LEDs, $96 \mathrm{~h}$

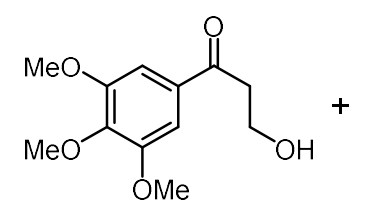

$2 e$<smiles>COc1ccccc1O</smiles>

$3 a$

Condition A: violet LED strips, 96 h, 2c (56 mg, 93\% yield) and 3a (27 mg, 88\% yield). 


\section{Organocatalytic photochemical reductive $\mathrm{C}-\mathrm{O}$ bond cleavage of 1 a and $\mathbf{1 b}$ in continuous flow}<smiles>COc1ccc(C(=O)C(CO)Oc2ccccc2OC)cc1</smiles>

$1 \mathrm{~b}$

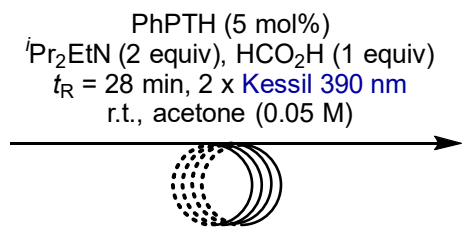<smiles>COc1ccc(C(=O)CC[OH2+])cc1</smiles>

$2 b$<smiles>COc1ccccc1O</smiles>

3a

Substrates $\mathbf{1 b}$ (76 mg, $0.25 \mathrm{mmol}$ ) were dissolved in acetone $(5 \mathrm{~mL})$, followed by adding the PhPTH photocatalyst (3.4 mg, $0.0125 \mathrm{mmol}, 5 \mathrm{~mol} \%)$, formic acid (10 $\mu \mathrm{L}, 0.25 \mathrm{mmol}, 1$ equiv.) and diisopropylethylamine $(87 \mu \mathrm{L}, 0.5$ mmol, 2 equiv.). The prepared solution was transferred to a 6 dram vial wrapped with aluminium foil and sparged with nitrogen for $5 \mathrm{~min}$ before irradiation. PFA tubing $(\mathrm{d}=0.03 \mathrm{in}, \mathrm{L}=10 \mathrm{~m})$ were coiled around $4 \times 25 \mathrm{~mL}$ test tubes. Two Kessil lamps (PR160-390 nm) where placed surrounding the tubing approximately $3 \mathrm{~cm}$ away from it (Figure S3). A peristaltic pump was used in order to control the flow of the reactor. To this prepared reaction mixture, one of the ends of the flow reactor tubing was inserted and the LEDs were turned on. A $0.1 \mathrm{~mL} / \mathrm{min}$ flow (residence time of $28 \mathrm{~min}$ ) was selected and the reaction was flowed. The resulting solution was collected in a 6 dram vial wrapped with aluminium foil at the end of the flow. After the reaction, the reaction mixture was concentrated under reduced pressure and dried in vacuo. The crude residue was purified by column chromatography to afford the desired products $\mathbf{2 b}$ (38 $\mathrm{mg}, 85 \%$ yield) and $\mathbf{3 a}(21 \mathrm{mg}, 67 \%$ yield).
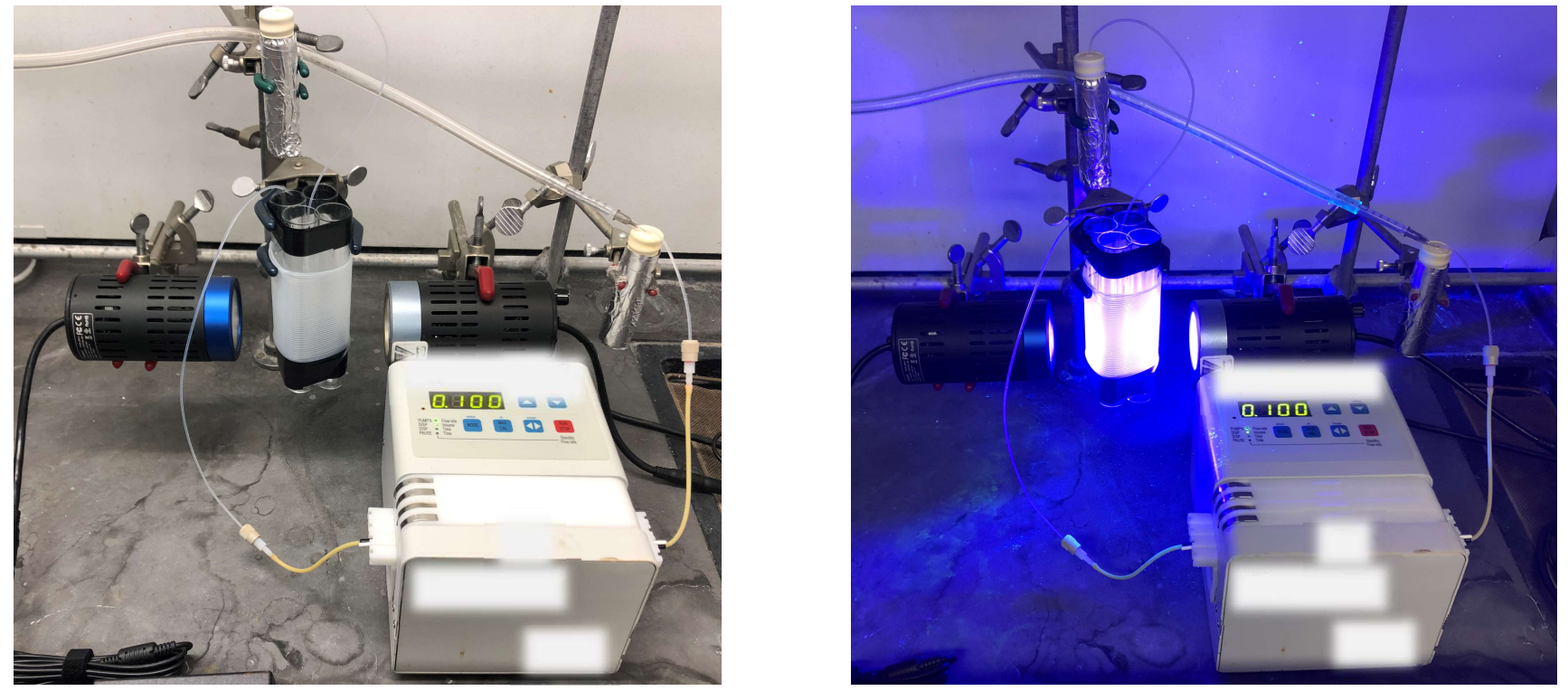

Figure S3. Flow reaction setup when light off (left) and light on (right). 


\section{Organocatalytic photochemical reductive $\mathrm{C}-\mathrm{O}$ bond cleavage of $1 \mathrm{a}(1 \mathrm{mmol})$ in continuous flow}

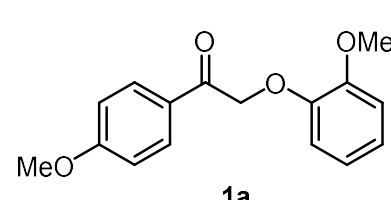

$1 \mathrm{a}$

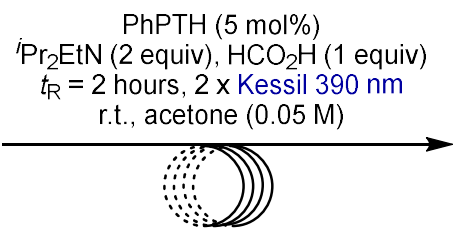

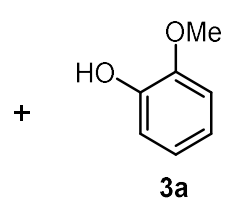

A scaled-up reaction of $1 \mathbf{a}$ was performed. Substrates $\mathbf{1 a}(272 \mathrm{mg}, 1 \mathrm{mmol})$ were dissolved in acetone $(20 \mathrm{~mL})$, followed by adding the PhPTH photocatalyst (13.8 $\mathrm{mg}, 0.05 \mathrm{mmol}, 5 \mathrm{~mol} \%)$, formic acid (38 $\mu \mathrm{L}, 1 \mathrm{mmol}, 1$ equiv.) and diisopropylethylamine ( $349 \mu \mathrm{L}, 2$ mmol, 2 equiv.). The prepared solution was transferred to a 6 dram vial wrapped with aluminium foil and sparged with nitrogen for $5 \mathrm{~min}$ before irradiation. PFA tubing $(\mathrm{d}=0.03$ in, $\mathrm{L}=$ $10 \mathrm{~m}$ ) were coiled around 4 × $25 \mathrm{~mL}$ test tubes. Two Kessil lamps (PR160-390 nm) where placed surrounding the tubing approximately $3 \mathrm{~cm}$ away from it. A peristaltic pump was used in order to control the flow of the reactor. To this prepared reaction mixture, one of the ends of the flow reactor tubing was inserted and the LEDs were turned on. A $0.1 \mathrm{~mL} / \mathrm{min}$ flow (residence time of $2 \mathrm{~h}$ ) was selected and the reaction was flowed. The resulting solution was collected in a 6 dram vial wrapped with aluminium foil at the end of the flow. After the reaction, the reaction mixture was concentrated under reduced pressure and dried in vacuo. The crude residue was purified by column chromatography to afford the desired products $\mathbf{2 a}$ (117 $\mathrm{mg}, 78 \%$ yield) and $\mathbf{3 a}$ ( $80 \mathrm{mg}, 65 \%$ yield). 


\section{One-pot, two-step approaches to lignin fragmentation}

\section{General procedures}
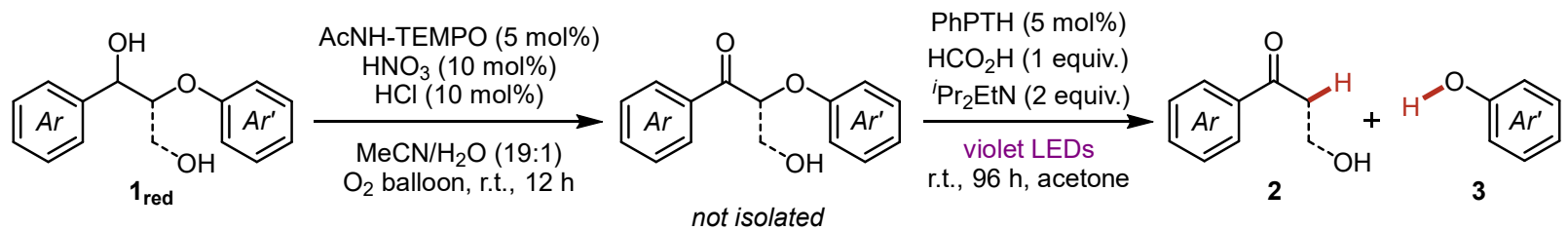

Method A. A vial equipped with a stir bar was charged with the lignin model substrates $\mathbf{1}_{\text {red }}(0.25 \mathrm{mmol}), 5 \mathrm{~mol} \%$ of 4-acetamido-TEMPO $(0.0125 \mathrm{mmol}, 5 \mathrm{~mol} \%)$ and acetonitrile $(0.5 \mathrm{~mL})$. Then, nitric acid $(67 \%, 0.025 \mathrm{mmol}, 10$ mol\% in $0.25 \mathrm{~mL}$ acetonitrile) and hydrochloric acid (37\%, $0.025 \mathrm{mmol}, 10 \mathrm{~mol} \%$ in $0.25 \mathrm{~mL}$ acetonitrile) and water $(0.05 \mathrm{~mL})$ were added. The resulting solution was sparged with oxygen gas and stirred for $12 \mathrm{~h}$ at room temperature under an oxygen balloon before evaporating solvent under reduced pressure. To the crude oxidation product, PhPTH (0.0125 mmol, $5 \mathrm{~mol} \%)$, formic acid (0.25 mmol, 1 equiv.), diisopropylethylamine $(0.5 \mathrm{mmol}, 2$ equiv.) and acetone $(5 \mathrm{~mL})$ were added without any purification. The resulting solution was sparged with nitrogen for $5 \mathrm{~min}$ before irradiated with violet LED strips at ambient temperature (Condition A). After $96 \mathrm{~h}$ of irradiation, the reaction mixture was concentrated under reduced pressure and dried in vacuo. The crude residue was purified by column chromatography to afford the desired products $\mathbf{2}$ and $\mathbf{3}$.
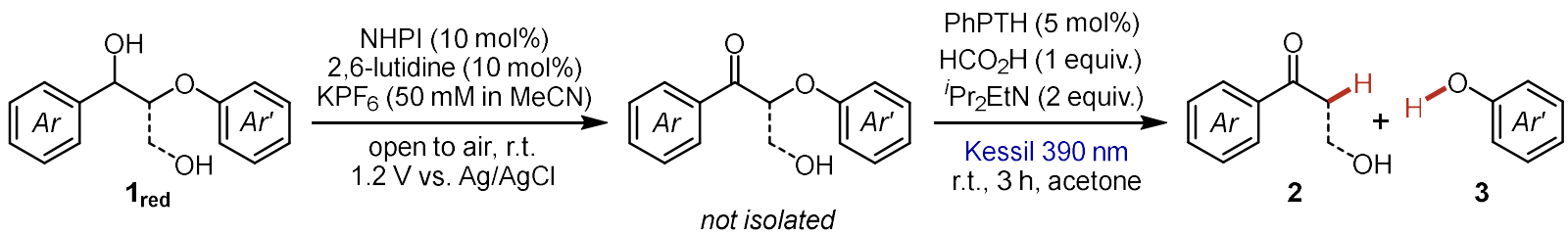

Method B. Lignin model substrates $\mathbf{1}_{\text {red }}(0.25 \mathrm{mmol}), N$-hydroxyphthalimide $(0.025 \mathrm{mmol}, 10 \mathrm{~mol} \%)$ and $2,6-$ lutidine $(0.025 \mathrm{mmol}, 10 \mathrm{~mol} \%)$ were added to a solution of $0.1 \mathrm{M} \mathrm{KPF}_{6}$ in acetonitrile $(5 \mathrm{~mL})$. The reaction solution was sparged with oxygen for 5 minutes, and then transferred to an undivided cell equipped with two RVC electrodes (anode and cathode) and a $\mathrm{Ag} / \mathrm{AgCl}(3 \mathrm{M} \mathrm{KCl})$ reference electrode. Open air electrolysis was carried out at a constant potential of $1.1 \mathrm{~V}$ vs $\mathrm{Ag} / \mathrm{AgCl}$ until reaction completion. The solvent was evaporated under reduced pressure. Without any purification, $\mathrm{PhPTH}(0.0125 \mathrm{mmol}, 5 \mathrm{~mol} \%)$, formic acid $(0.25 \mathrm{mmol}, 1$ equiv.), diisopropylethylamine $(0.5 \mathrm{mmol}, 2$ equiv. $)$ and acetone $(5 \mathrm{~mL})$ were directly added. The resulting solution was sparged with nitrogen for 5 min before irradiated with Kessil $390 \mathrm{~nm}$ at ambient temperature (Condition B). After $3 \mathrm{~h}$ of irradiation, the reaction mixture was concentrated under reduced pressure and dried in vacuo. The crude residue was purified by column chromatography to afford the desired products 2 and $\mathbf{3}$. 


\section{Reaction results}<smiles>COc1ccc(C(O)COc2ccccc2OC)cc1</smiles>
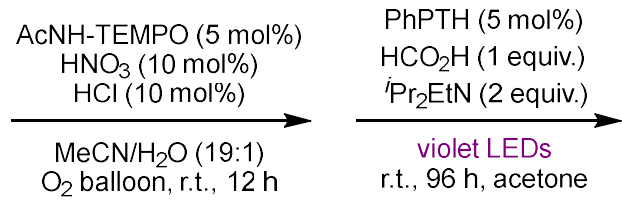<smiles>COc1ccc(C(C)=O)cc1</smiles>

2a<smiles>COc1ccccc1O</smiles>

3a

Substrate 1 $\mathbf{a}_{\text {red }}$ was subjected to Method A. The reaction afforded 2a (35 mg, 92\% yield) and 3a (28 mg, 89\% yield).
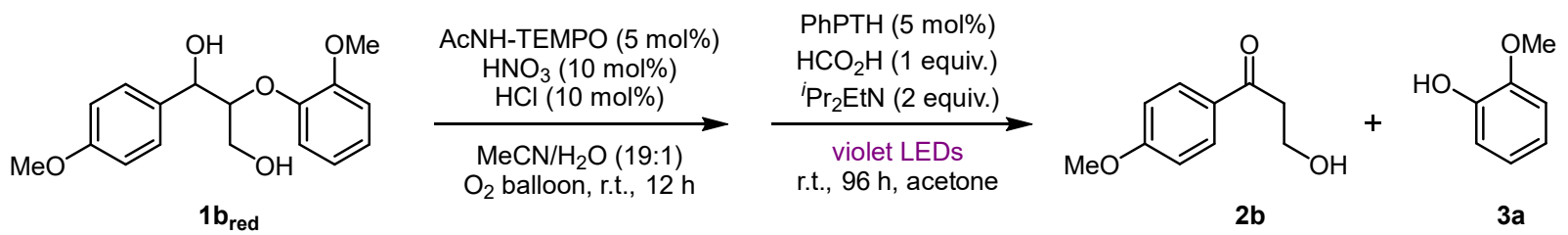

Substrate $\mathbf{1} \mathbf{b}_{\text {red }}$ was subjected to Method A. The reaction afforded $\mathbf{2 b}$ (41 mg, 90\% yield) and 3a (28 mg, $91 \%$ yield).<smiles>COc1ccc(C(O)C(CO)Oc2ccccc2OC)cc1</smiles>

$1 b_{\text {red }}$

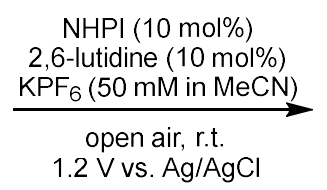

$1.2 \mathrm{Vvs} . \mathrm{Ag} / \mathrm{AgC}$

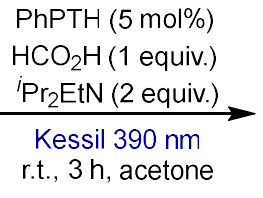

r.t., $3 \mathrm{~h}$, acetone

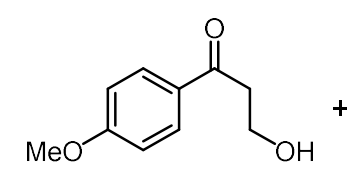

$2 \mathrm{~b}$<smiles>COc1ccccc1O</smiles>

$3 a$

Substrate $\mathbf{1} \mathbf{b}_{\text {red }}$ was subjected to Method B. The reaction afforded $\mathbf{2 b}$ (39 mg, 87\% yield) and $\mathbf{3 a}$ ( $29 \mathrm{mg}, 80 \%$ yield).<smiles>COc1ccc(C(O)COc2ccccc2OC)cc1OC</smiles>

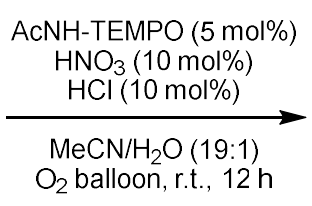

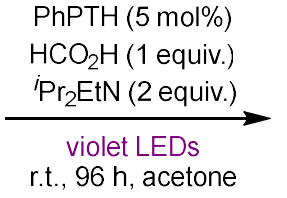

r.t. $96 \mathrm{~h}$, acetone

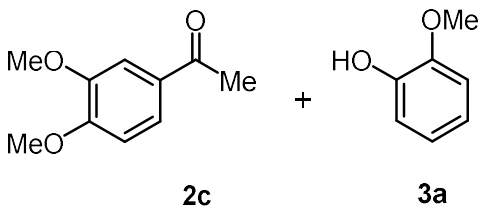

$3 a$

Substrate $\mathbf{1} \mathbf{c}_{\text {red }}$ was subjected to Method A. The reaction afforded $\mathbf{2 c}$ (41 mg, 91\% yield) and $\mathbf{3 a}$ (27 mg, 88\% yield). 

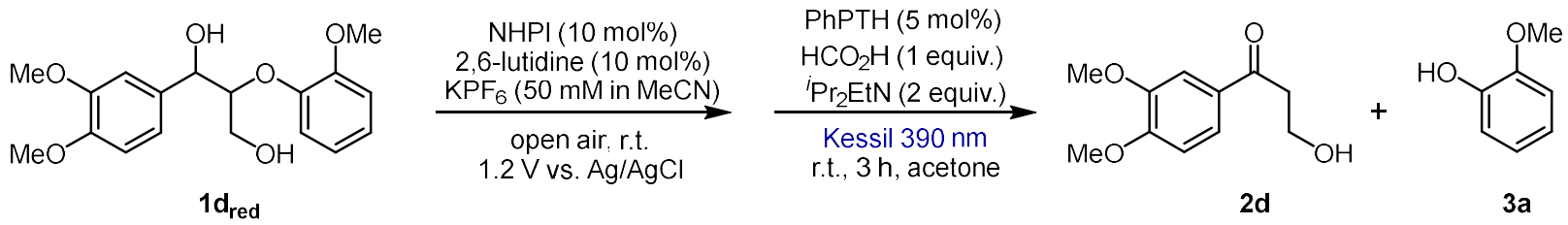

Substrate $\mathbf{1} \mathbf{d}_{\text {red }}$ was subjected to Method B. The reaction afforded $2 \mathbf{d}$ (49 mg, 93\% yield) and 3a (21 mg, 68\% yield).
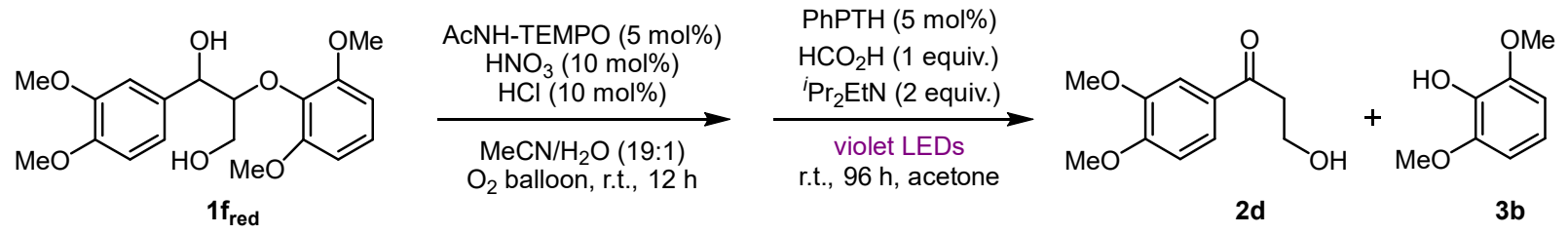

Substrate $\mathbf{1} \mathbf{f}_{\text {red }}$ was subjected to Method A. The reaction afforded $\mathbf{2 d}$ (45 mg, 86\% yield) and $\mathbf{3 b}$ (35 $\mathrm{mg}, 90 \%$ yield).
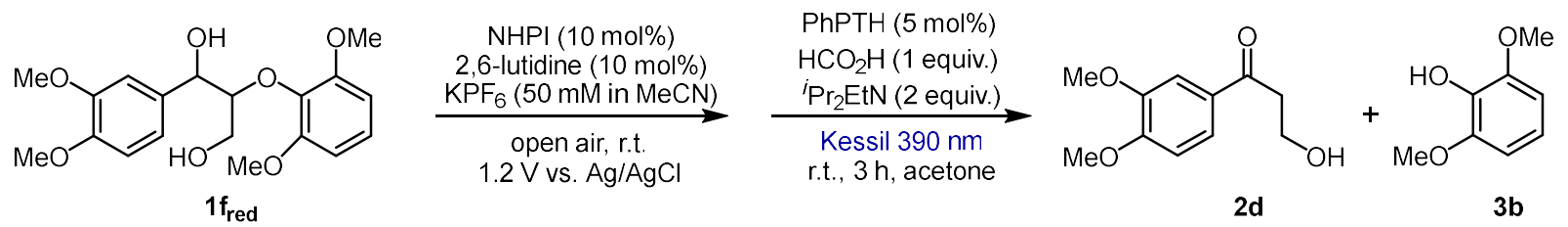

Substrate $\mathbf{1} \mathbf{f}_{\text {red }}$ was subjected to Method B. The reaction afforded $\mathbf{2 d}$ (50 mg, 95\% yield) and $\mathbf{3 b}$ (22 mg, 56\% yield).
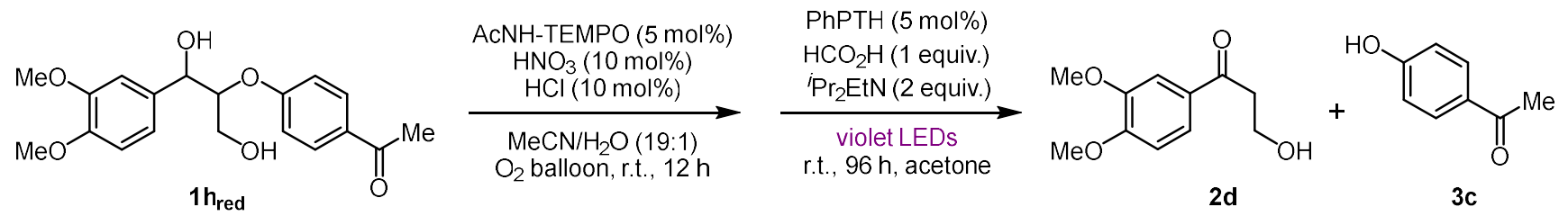

Substrate $\mathbf{1} \mathbf{h}_{\text {red }}$ was subjected to Method A. The reaction afforded $\mathbf{2 d}(40 \mathrm{mg}, 76 \%$ yield) and $\mathbf{3 c}$ ( $27 \mathrm{mg}, 78 \%$ yield). 


\section{Characterization of products $2 a-2 e$ and $3 a-3 b$}<smiles>COc1ccc(C(C)=O)cc1</smiles>

\section{1-(4-Methoxyphenyl)ethan-1-one (2a)}

White solid; $\mathbf{R}_{\mathbf{f}}=0.6$ (hexanes/EtOAc 3:2); ${ }^{1} \mathbf{H}$ NMR (500 MHz, $\left.\mathbf{C D C l}_{3}\right) \delta 7.92(\mathrm{~d}, J=5 \mathrm{~Hz}, 2 \mathrm{H}), 6.92(\mathrm{~d}, J=10$ $\mathrm{Hz}, 2 \mathrm{H}), 3.85$ (s, 3H), 2.54 (s, 3H); ${ }^{13} \mathbf{C}$ NMR (126 MHz, $\left.\mathbf{C D C l}_{3}\right) \delta$ 196.7, 163.4, 130.5, 130.2, 113.6, 55.5, 26.4; HRMS (ESI) $m / z$ : $[\mathrm{M}+\mathrm{H}]^{+}$Calcd for $\mathrm{C}_{9} \mathrm{H}_{11} \mathrm{O}_{2}$ 151.0754; Found 151.0754.<smiles>COc1ccc(C(=O)CCO)cc1</smiles>

\section{3-Hydroxy-1-(4-methoxyphenyl)propan-1-one (2b)}

White solid; $\mathbf{R}_{\mathbf{f}}=0.4$ (hexanes/EtOAc 2:3); ${ }^{1} \mathbf{H}$ NMR (500 MHz, $\left.\mathbf{C D C l}_{3}\right) \delta 7.94(\mathrm{~d}, J=15 \mathrm{~Hz}, 2 \mathrm{H}), 6.94(\mathrm{~d}, J=15$ $\mathrm{Hz}, 2 \mathrm{H}), 4.01(\mathrm{q}, J=10,5 \mathrm{~Hz}, 2 \mathrm{H}), 3.88(\mathrm{~s}, 3 \mathrm{H}), 3.18(\mathrm{t}, J=5 \mathrm{~Hz}, 2 \mathrm{H}), 2.76(\mathrm{t}, J=5 \mathrm{~Hz}, 1 \mathrm{H}) ;{ }^{13} \mathbf{C} \mathbf{N M R}(\mathbf{1 2 6} \mathbf{M H z}$, $\left.\mathbf{C D C l}_{3}\right) \delta 199.2,164.0,130.5,129.9,114.0,58.4,55.7,40.2$; HRMS (ESI) $m / z$ : $[\mathrm{M}+\mathrm{Na}]^{+} \mathrm{Calcd}$ for $\mathrm{C}_{10} \mathrm{H}_{12} \mathrm{NaO}_{3}$ 203.0679; Found 203.0677.<smiles>COc1ccc(C(C)=O)cc1OC</smiles>

\section{1-(3,4-Dimethoxyphenyl)ethan-1-one (2c)}

White solid; $\mathbf{R}_{\mathbf{f}}=0.4$ (hexanes/EtOAc 3:2); ${ }^{1} \mathbf{H}$ NMR (500 MHz, $\left.\mathbf{C D C l}_{3}\right) \delta 7.55(\mathrm{dd}, J=10,5 \mathrm{~Hz}, 1 \mathrm{H}), 7.51(\mathrm{~s}$, 1H), 6.87 (d, $J=10 \mathrm{~Hz}, 1 \mathrm{H}), 3.93$ (s, 3H), 3.92 (s, 3H), 2.55 (s, 3H); ${ }^{13} \mathbf{C}$ NMR (126 MHz, CDCl $) \delta$ 196.6, 153.1, 148.7, 130.2, 123.0, 109.7, 55.8, 55.7, 26.1; HRMS (ESI) $\mathrm{m} / \mathrm{z}:[\mathrm{M}+\mathrm{H}]^{+}$Calcd for $\mathrm{C}_{10} \mathrm{H}_{13} \mathrm{O}_{3}$ 181.0859; Found 181.0858 .<smiles>COc1ccc(C(=O)CCO)cc1OC</smiles>

\section{1-(3,4-Dimethoxyphenyl)-3-hydroxypropan-1-one (2d)}

White solid; $\mathbf{R}_{\mathbf{f}}=0.3$ (hexanes/EtOAc 2:3); ${ }^{1} \mathbf{H}$ NMR (500 MHz, $\left.\mathbf{C D C l}_{3}\right) \delta 7.59$ (d, $\left.J=5 \mathrm{~Hz}, 1 \mathrm{H}\right), 7.52(\mathrm{~s}, 1 \mathrm{H})$, $6.90(\mathrm{~d}, J=5 \mathrm{~Hz}, 1 \mathrm{H}), 4.02(\mathrm{t}, J=5 \mathrm{~Hz}, 2 \mathrm{H}), 3.95(\mathrm{~s}, 3 \mathrm{H}), 3.94(\mathrm{~s}, 3 \mathrm{H}), 3.19(\mathrm{t}, J=5 \mathrm{~Hz}, 2 \mathrm{H}), 2.72(\mathrm{br} \mathrm{s}, 1 \mathrm{H}) ;{ }^{13} \mathrm{C}$ NMR (126 MHz, CDCl $) \delta 198.1,152.6,148.0,128.9,121.9,109.0,108.8,57.3,55.1,55.0,38.8$; HRMS (ESI) $m / z:[\mathrm{M}+\mathrm{H}]^{+}$Calcd for $\mathrm{C}_{11} \mathrm{H}_{15} \mathrm{O}_{4} 211.0965$; Found 211.0968 . 


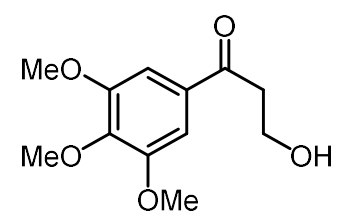

3-Hydroxy-1-(3,4,5-trimethoxyphenyl)propan-1-one (2e)

White solid; $\mathbf{R}_{\mathbf{f}}=0.2$ (hexanes/EtOAc 2:3); ${ }^{1} \mathbf{H}$ NMR (500 MHz, $\left.\mathbf{C D C l}_{3}\right) \delta 7.23(\mathrm{~s}, 2 \mathrm{H}), 4.05-4.02(\mathrm{~m}, 2 \mathrm{H}), 3.93$ (s, 9H), $3.21(\mathrm{t}, J=5 \mathrm{~Hz}, 2 \mathrm{H}), 2.61(\mathrm{t}, J=5 \mathrm{~Hz}, 1 \mathrm{H}) ;{ }^{13} \mathbf{C}$ NMR (126 MHz, $\left.\mathbf{C D C l}_{3}\right) \delta 199.2,153.1,143.0,131.9$, 105.6, 60.9, 58.2, 56.3, 40.1; HRMS (ESI) $m / z$ : $[\mathrm{M}+\mathrm{Na}]^{+}$Calcd for $\mathrm{C}_{12} \mathrm{H}_{16} \mathrm{NaO}_{5}$ 263.0890; Found 263.0892.<smiles>COc1ccccc1O</smiles>

\section{2-Methoxyphenol (3a)}

Light yellow oil; $\mathbf{R}_{\mathbf{f}}=0.6$ (hexanes/EtOAc 2:1); ${ }^{1} \mathbf{H}$ NMR (500 MHz, $\left.\mathbf{C D C l}_{3}\right) \delta 6.95-6.91(\mathrm{~m}, 1 \mathrm{H}), 6.88(\mathrm{dd}, J=$ 5.9, 3.2 Hz, 1H), 6.87-6.85 (m, 2H), 5.60 (br, 1H), 3.89 (s, 3H); ${ }^{13} \mathbf{C}$ NMR (126 MHz, CDCl $) \delta$ 146.7, 145.7, 121.5, 120.2, 114.7, 110.9, 55.9; HRMS (ESI) $m / z:[\mathrm{M}+\mathrm{H}]^{+}$Calcd for $\mathrm{C}_{7} \mathrm{H}_{9} \mathrm{O}_{2}$ 125.0597; Found 125.0595.

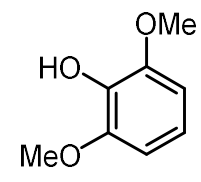

\section{2,6-Dimethoxyphenol (3b)}

Off-white solid; $\mathbf{R}_{\mathbf{f}}=0.4$ (hexanes/EtOAc 2:1); ${ }^{1} \mathbf{H}$ NMR (500 MHz, $\left.\mathbf{C D C l}_{3}\right) \delta 6.79(\mathrm{t}, J=8.4 \mathrm{~Hz}, 1 \mathrm{H}), 6.58(\mathrm{~d}, J$ $=8.5 \mathrm{~Hz}, 2 \mathrm{H}), 5.55$ (br, 1H), 3.87 (s, 6H); ${ }^{13} \mathbf{C}$ NMR (126 MHz, $\left.\mathbf{C D C l}_{3}\right) \delta 147.3,134.9,119.1,105.0,56.3$; HRMS (ESI) $m / z:[\mathrm{M}+\mathrm{H}]^{+}$Calcd for $\mathrm{C}_{8} \mathrm{H}_{11} \mathrm{O}_{3}$ 155.0703; Found 155.0702 .<smiles>CC(=O)c1ccc(O)cc1</smiles>

\section{1-(4-Hydroxyphenyl)ethan-1-one (3c)}

White solid; $\mathbf{R}_{\mathbf{f}}=0.4$ (hexanes/EtOAc 3:2); ${ }^{1} \mathbf{H}$ NMR (500 MHz, $\left.\mathbf{C D C l}_{3}\right) \delta 8.38(\mathrm{~s}, 1 \mathrm{H}), 7.92(\mathrm{~d}, J=10 \mathrm{MHz}, 2 \mathrm{H})$, 6.97 (d, $J=10$ MHz, 2H), 2.60 (s, 3H); ${ }^{13} \mathbf{C}$ NMR (126 MHz, CDCl $) \delta$ 199.1, 161.7, 131.3, 129.2, 115.6, 26.3; HRMS (ESI) $m / z$ : $[\mathrm{M}+\mathrm{H}]^{+}$Calcd for $\mathrm{C}_{8} \mathrm{H}_{9} \mathrm{O}_{2}$ 137.0597; Found 137.0594. 


\section{Characterization of substrates $1 \mathrm{a}-1 \mathrm{i}, 1 \mathrm{a}_{\text {red-1 }}-1 \mathrm{~d}_{\text {red }}, 1 \mathrm{f}_{\text {red, }}$ and $1 \mathrm{~h}_{\text {red }}$}

All starting materials were prepared according to ref. S1. Characterizations are listed below.<smiles>COc1ccc(C(=O)COc2ccccc2OC)cc1</smiles>

\section{2-(2-Methoxyphenoxy)-1-(4-methoxyphenyl)ethan-1-one (1a)}

White solid; $\mathbf{R}_{\mathbf{f}}=0.5$ (hexanes/EtOAc 3:2); ${ }^{1} \mathbf{H}$ NMR (500 $\left.\mathbf{~ M H z}, \mathbf{C D C l}_{3}\right) \delta 8.01(\mathrm{~d}, J=8.6 \mathrm{~Hz}, 2 \mathrm{H}), 6.98-6.82(\mathrm{~m}$, 6H), 5.27 (s, 2H), 3.87 (s, 3H), 3.86 (s, 3H); ${ }^{13} \mathbf{C}$ NMR (126 MHz, CDCl $) \delta$ 193.0, 163.9, 149.7, 147.6, 130.4, 127.6, 122.2, 120.7, 114.7, 113.9, 112.1, 71.9, 55.8, 55.4; HRMS (ESI) $\mathrm{m} / z:[\mathrm{M}+\mathrm{Na}]^{+} \mathrm{Calcd}$ for $\mathrm{C}_{16} \mathrm{H}_{16} \mathrm{NaO}_{4}$ 295.0941; Found 295.0937.<smiles>COc1ccc(C(=O)C(CO)Oc2ccccc2OC)cc1</smiles>

3-Hydroxy-2-(2-methoxyphenoxy)-1-(4-methoxyphenyl)propan-1-one (1b)

White solid; $\mathbf{R}_{\mathbf{f}}=0.3$ (hexanes/EtOAc 1:2); ${ }^{1} \mathbf{H}$ NMR (500 $\left.\mathbf{~ M H z , ~} \mathbf{C D C l}_{3}\right) \delta 8.06(\mathrm{~d}, J=9.0 \mathrm{~Hz}, 2 \mathrm{H}), 7.00-6.77(\mathrm{~m}$, $6 \mathrm{H}), 5.37(\mathrm{t}, J=5.3 \mathrm{~Hz}, 1 \mathrm{H}), 4.05(\mathrm{t}, J=5.9 \mathrm{~Hz}, 2 \mathrm{H}), 3.85(\mathrm{~s}, 3 \mathrm{H}), 3.83(\mathrm{~s}, 3 \mathrm{H}), 3.24(\mathrm{t}, J=6.6 \mathrm{~Hz}, 1 \mathrm{H}) ;{ }^{13} \mathbf{C} \mathbf{N M R}$ (126 MHz, CDCl $) \delta$ 195.0, 164.0, 150.4, 146.9, 131.2, 127.9, 123.5, 121.1, 118.5, 113.9, 112.3, 84.6, 63.5, 55.8, 55.5; HRMS (ESI) $m / z$ : $[\mathrm{M}+\mathrm{Na}]^{+}$Calcd for $\mathrm{C}_{17} \mathrm{H}_{18} \mathrm{NaO}_{5}$ 325.1046; Found 325.1046.<smiles>COc1ccc(C(=O)COc2ccccc2OC)cc1OC</smiles>

\section{1-(3,4-Dimethoxyphenyl)-2-(2-methoxyphenoxy)ethan-1-one (1c)}

White solid; $\mathbf{R}_{\mathbf{f}}=0.4$ (hexanes/EtOAc 3:2); ${ }^{1} \mathbf{H}$ NMR (500 MHz, $\left.\mathbf{C D C l}_{3}\right) \delta 7.65(\mathrm{~d}, J=8.3 \mathrm{~Hz}, 1 \mathrm{H}), 7.58(\mathrm{~s}, 1 \mathrm{H})$, 6.97-6.79 (m, 5H), 5.27 (s, 2H), $3.92(\mathrm{~s}, 3 \mathrm{H}), 3.91(\mathrm{~s}, 3 \mathrm{H}), 3.86(\mathrm{~s}, 3 \mathrm{H}) ;{ }^{\mathbf{1 3}} \mathbf{C} \mathbf{~ N M R}\left(\mathbf{1 2 6} \mathbf{~ M H z}, \mathbf{C D C l}_{3}\right) \delta 193.1$, 153.7, 149.6, 149.1, 147.5, 127.7, 122.7, 122.2, 120.7, 114.6, 112.0, 110.3, 110.0, 71.9, 56.0, 55.9, 55.8; HRMS (ESI) $m / z:[\mathrm{M}+\mathrm{H}]^{+}$Calcd for $\mathrm{C}_{17} \mathrm{H}_{19} \mathrm{O}_{5} 303.1227$; Found 303.1228 . 
<smiles>COc1ccc(C(=O)C(CO)Oc2ccccc2OC)cc1OC</smiles>

\section{1-(3,4-Dimethoxyphenyl)-3-hydroxy-2-(2-methoxyphenoxy)propan-1-one (1d)}

White solid; $\mathbf{R}_{\mathbf{f}}=0.3$ (hexanes/EtOAc 1:2); ${ }^{1} \mathbf{H}$ NMR (500 MHz, $\left.\mathbf{C D C l}_{3}\right) \delta 7.74(\mathrm{~d}, J=8.4 \mathrm{~Hz}, 1 \mathrm{H}), 7.60(\mathrm{~s}, 1 \mathrm{H})$, 6.98-6.76 (m, 5H), 5.39 (t, $J=5.3 \mathrm{~Hz}, 1 \mathrm{H}), 4.07$ (q, $J=5.7,4.8 \mathrm{~Hz}, 2 \mathrm{H}), 3.91(\mathrm{~s}, 3 \mathrm{H}), 3.88(\mathrm{~s}, 3 \mathrm{H}), 3.82(\mathrm{~s}, 3 \mathrm{H})$; ${ }^{13}$ C NMR (126 MHz, CDCl $) \delta$ 195.0, 153.8, 150.3, 149.1, 146.9, 128.0, 123.6, 123.3, 121.1, 118.0, 112.3, 111.0, 110.1, 84.3, 63.6, 56.0, 55.9, 55.7; HRMS (ESI) $m / z$ : $[\mathrm{M}+\mathrm{Na}]^{+}$Calcd for $\mathrm{C}_{18} \mathrm{H}_{20} \mathrm{NaO}_{6} 355.1152$; Found 355.1152.<smiles>COc1ccc(C(=O)COc2c(OC)cccc2OC)cc1OC</smiles>

\section{2-(2,6-Dimethoxyphenoxy)-1-(3,4-dimethoxyphenyl)ethan-1-one (1e)}

White solid; $\mathbf{R}_{\mathbf{f}}=0.5$ (hexanes/EtOAc 3:2); ${ }^{1} \mathbf{H}$ NMR (500 MHz, $\left.\mathbf{C D C l}_{3}\right) \delta 7.71(\mathrm{~d}, J=8.4 \mathrm{~Hz}, 1 \mathrm{H}), 7.64(\mathrm{~s}, 1 \mathrm{H})$, 7.03-6.96 (m, 1H), $6.88(\mathrm{~d}, J=8.4 \mathrm{~Hz}, 1 \mathrm{H}), 6.57$ (d, $J=8.4 \mathrm{~Hz}, 2 \mathrm{H}), 5.13(\mathrm{~s}, 2 \mathrm{H}), 3.93(\mathrm{~s}, 6 \mathrm{H}), 3.80(\mathrm{~s}, 6 \mathrm{H}) ;{ }^{13} \mathbf{C}$ NMR (126 MHz, $\left.\mathbf{C D C l}_{3}\right) \delta$ 193.6, 153.3, 153.2, 148.9, 136.6, 128.3, 124.0, 123.0, 110.6, 110.0, 105.3, 75.2, 56.02, 55.98, 55.96; HRMS (ESI) $m / z:[\mathrm{M}+\mathrm{H}]^{+}$Calcd for $\mathrm{C}_{18} \mathrm{H}_{21} \mathrm{O}_{6}$ 333.1333; Found 333.1333.<smiles>COCC(Oc1c(OC)cccc1OC)C(=O)c1ccc(OC)c(OC)c1</smiles>

\section{2-(2,6-Dimethoxyphenoxy)-1-(3,4-dimethoxyphenyl)-3-hydroxypropan-1-one (1f)}

White solid; $\mathbf{R}_{\mathbf{f}}=0.2$ (hexanes/EtOAc 1:2); ${ }^{1} \mathbf{H}$ NMR (500 $\left.\mathbf{M H z}, \mathbf{C D C l}_{3}\right) \delta$ 7.73-7.69 (m, 1H), 7.66-7.62 (m, 1H), $6.99(\mathrm{t}, J=8.4 \mathrm{~Hz}, 1 \mathrm{H}), 6.86(\mathrm{~d}, J=8.4 \mathrm{~Hz}, 1 \mathrm{H}), 6.55(\mathrm{~d}, J=8.4 \mathrm{~Hz}, 2 \mathrm{H}), 5.07(\mathrm{dd}, J=7.7,3.0 \mathrm{~Hz}, 1 \mathrm{H}), 3.97(\mathrm{~d}, J$ $=11.5 \mathrm{~Hz}, 1 \mathrm{H}), 3.91(\mathrm{~d}, J=2.8 \mathrm{~Hz}, 6 \mathrm{H}), 3.82(\mathrm{~d}, J=8.9 \mathrm{~Hz}, 1 \mathrm{H}), 3.70(\mathrm{~s}, 6 \mathrm{H}) ;{ }^{13} \mathbf{C ~ N M R}\left(\mathbf{1 2 6} \mathbf{M H z}, \mathbf{C D C l}_{3}\right) \delta$ 194.8, 153.4, 152.6, 149.0, 136.6, 128.6, 124.1, 123.3, 110.9, 109.9, 105.2, 87.4, 63.5, 55.9, 55.90, 55.8; HRMS (ESI) $m / z:[\mathrm{M}+\mathrm{Na}]^{+}$Calcd for $\mathrm{C}_{19} \mathrm{H}_{22} \mathrm{NaO}_{7}$ 385.1258; Found 385.1257. 
<smiles>COc1ccc(C(=O)COc2ccc(C(C)=O)cc2)cc1OC</smiles>

2-(4-Acetylphenoxy)-1-(3,4-dimethoxyphenyl)ethan-1-one (1g)

White solid; $\mathbf{R}_{\mathbf{f}}=0.3$ (hexanes/EtOAc 3:2); ${ }^{1} \mathbf{H}$ NMR (500 MHz, $\left.\mathbf{C D C l}_{3}\right) \delta 7.92(\mathrm{~d}, J=8.7 \mathrm{~Hz}, 2 \mathrm{H}), 7.63(\mathrm{~d}, J=$ $8.4 \mathrm{~Hz}, 1 \mathrm{H}), 7.55(\mathrm{~s}, 1 \mathrm{H}), 6.96(\mathrm{~d}, J=8.7 \mathrm{~Hz}, 2 \mathrm{H}), 6.91(\mathrm{~d}, J=8.4 \mathrm{~Hz}, 1 \mathrm{H}), 5.31(\mathrm{~s}, 2 \mathrm{H}), 3.96(\mathrm{~s}, 3 \mathrm{H}), 3.93(\mathrm{~s}, 3 \mathrm{H})$, 2.54 (s, 3H); ${ }^{13} \mathbf{C}$ NMR (126 MHz, $\left.\mathbf{C D C l}_{3}\right) \delta$ 196.6, 192.1, 161.9, 154.1, 149.4, 131.0, 130.6, 127.4, 122.7, 114.4, 110.22, 110.16, 70.3, 56.1, 56.0, 26.3; HRMS (ESI) $m / z$ : $[\mathrm{M}+\mathrm{H}]^{+}$Calcd for $\mathrm{C}_{18} \mathrm{H}_{19} \mathrm{O}_{5}$ 315.1227; Found 315.1225.<smiles>COc1ccc(C(=O)C(CO)Oc2ccc(C(C)=O)cc2)cc1OC</smiles>

2-(4-Acetylphenoxy)-1-(3,4-dimethoxyphenyl)-3-hydroxypropan-1-one (1 h)

Yellow solid; $\mathbf{R}_{\mathbf{f}}=0.21$ (hexanes/EtOAc 1:2); ${ }^{1} \mathbf{H}$ NMR (500 MHz, $\left.\mathbf{C D C l}_{3}\right) \delta 7.88(\mathrm{~d}, J=8.8 \mathrm{~Hz}, 2 \mathrm{H}), 7.74$ (dd, $J$ $=8.4,1.8 \mathrm{~Hz}, 1 \mathrm{H}), 7.55(\mathrm{~d}, J=1.8 \mathrm{~Hz}, 1 \mathrm{H}), 6.91(\mathrm{~d}, J=8.8 \mathrm{~Hz}, 2 \mathrm{H}), 6.90(\mathrm{~d}, J=8.4 \mathrm{~Hz}, 1 \mathrm{H}), 5.63(\mathrm{dd}, J=6.2,3.9$ $\mathrm{Hz}, 1 \mathrm{H}), 4.20$ (dd, $J=12.2,3.9 \mathrm{~Hz}, 1 \mathrm{H}), 4.15(\mathrm{dd}, J=12.2,6.2 \mathrm{~Hz}, 1 \mathrm{H}), 3.96(\mathrm{~s}, 3 \mathrm{H}), 3.90(\mathrm{~s}, 3 \mathrm{H}), 2.52$ (s, 3H); ${ }^{13}$ C NMR (126 MHz, CDCl $) \delta$ 196.8, 194.0, 161.2, 154.6, 149.6, 131.4, 130.9, 127.6, 123.6, 115.0, 110.9, 110.4, 81.1, 63.7, 56.3, 56.2, 26.5; HRMS (ESI) $\mathrm{m} / \mathrm{z}:[\mathrm{M}+\mathrm{H}]^{+}$Calcd for $\mathrm{C}_{19} \mathrm{H}_{21} \mathrm{O}_{6}$ 345.1333; Found 345.1332.<smiles>COc1ccccc1OC(CO)C(=O)c1cc(OC)c(OC)c(OC)c1</smiles>

\section{3-Hydroxy-2-(2-methoxyphenoxy)-1-(3,4,5-trimethoxyphenyl)propan-1-one (1i)}

White solid; $\mathbf{R}_{\mathbf{f}}=0.2$ (hexanes/EtOAc 1:2); ${ }^{1} \mathbf{H}$ NMR (500 MHz, $\left.\mathbf{C D C l}_{3}\right) \delta 7.36(\mathrm{~s}, 2 \mathrm{H}), 7.05-6.99(\mathrm{~m}, 1 \mathrm{H}), 6.92$ $(\mathrm{ddd}, J=8.0,3.7,1.5 \mathrm{~Hz}, 2 \mathrm{H}), 6.84(\mathrm{td}, J=7.7,1.5 \mathrm{~Hz}, 1 \mathrm{H}), 5.39-5.32(\mathrm{~m}, 1 \mathrm{H}), 4.09(\mathrm{~d}, J=5.9 \mathrm{~Hz}, 2 \mathrm{H}), 3.92(\mathrm{~s}$, 3H), 3.88 (s, 6H), 3.85 (s, 3H); ${ }^{13} \mathbf{C}$ NMR (126 MHz, $\left.\mathbf{C D C l}_{3}\right) \delta$ 195.6, 153.1, 150.5, 146.8, 143.3, 130.0, 123.7, 121.2, 118.4, 112.3, 106.6, 84.6, 63.5, 61.0, 56.3, 55.8; HRMS (ESI) $m / z$ : $[\mathrm{M}+\mathrm{Na}]^{+}$Calcd for $\mathrm{C}_{19} \mathrm{H}_{22} \mathrm{NaO}_{7}$ 385.1258; Found 385.1257. 
<smiles>COc1ccc(C(O)COc2ccccc2OC)cc1</smiles>

2-(2-Methoxyphenoxy)-1-(4-methoxyphenyl)ethan-1-ol (1 $\left.\mathrm{a}_{\text {red }}\right)$

Colorless oil; $\mathbf{R}_{\mathbf{f}}=0.4\left(\right.$ hexanes/EtOAc 1:1); ${ }^{1} \mathbf{H}$ NMR (500 MHz, $\left.\mathbf{C D C l}_{3}\right) \delta 7.36(\mathrm{~d}, J=8.4 \mathrm{~Hz}, 2 \mathrm{H}), 7.02-6.97$ (m, 1H), 6.96-6.88 (m, 5H), $5.05(\mathrm{dd}, J=9.5,2.7 \mathrm{~Hz}, 1 \mathrm{H}), 4.15(\mathrm{dd}, J=10.1,2.7 \mathrm{~Hz}, 1 \mathrm{H}), 3.96(\mathrm{t}, J=9.8 \mathrm{~Hz}, 1 \mathrm{H})$, 3.89 (s, 3H), 3.81 (s, 3H), 3.34 (br, 1H); ${ }^{13} \mathbf{C}$ NMR (126 MHz, $\left.\mathbf{C D C l}_{3}\right) \delta 159.5,150.1,148.1,131.8,127.7,122.5$, 121.2, 115.7, 114.0, 112.1, 76.2, 72.0, 56.0, 55.4; HRMS (ESI) $m / z:[\mathrm{M}+\mathrm{Na}]^{+}$Calcd for $\mathrm{C}_{16} \mathrm{H}_{18} \mathrm{NaO}_{4}$ 297.1097; Found 297.1096.<smiles>COc1ccc(C(O)C(CO)Oc2ccccc2OC)cc1</smiles>

2-(2-Methoxyphenoxy)-1-(4-methoxyphenyl)propane-1,3-diol (1 $\left.b_{\text {red }}\right)$

Colorless oil; $\mathbf{R}_{\mathbf{f}}=0.2$ (hexanes/EtOAc 1:1); Major diastereomer (erythro): ${ }^{1} \mathbf{H} \mathbf{~ N M R}\left(\mathbf{5 0 0} \mathbf{~ M H z}, \mathbf{C D C l}_{3}\right) \delta 7.31$ $(\mathrm{d}, J=8.6 \mathrm{~Hz}, 2 \mathrm{H}), 7.09-7.04(\mathrm{~m}, 1 \mathrm{H}), 6.98(\mathrm{dd}, J=7.9,1.5 \mathrm{~Hz}, 1 \mathrm{H}), 6.96-6.87(\mathrm{~m}, 4 \mathrm{H}), 5.02-4.98(\mathrm{~m}, 1 \mathrm{H}), 4.18-$ $4.14(\mathrm{~m}, 1 \mathrm{H}), 3.95-3.91(\mathrm{~m}, 1 \mathrm{H}), 3.89(\mathrm{~s}, 3 \mathrm{H}), 3.80(\mathrm{~s}, 3 \mathrm{H}), 3.65(\mathrm{ddd}, J=12.2,7.6,3.3 \mathrm{~Hz}, 1 \mathrm{H}), 3.39(\mathrm{~d}, J=3.3$ $\mathrm{Hz}, 1 \mathrm{H}), 2.72(\mathrm{dd}, J=7.6,5.4 \mathrm{~Hz}, 1 \mathrm{H}) ;{ }^{13} \mathbf{C} \mathbf{N M R}\left(\mathbf{1 2 6} \mathbf{M H z}, \mathbf{C D C l}_{3}\right) \delta 159.2,151.7,147.0,132.2,127.4,124.3$, 121.7, 121.1, 113.9, 112.3, 87.4, 72.7, 60.8, 56.0, 55.4; Minor diastereomer (threo): ${ }^{1} \mathbf{H}$ NMR (500 MHz, $\left.\mathbf{C D C l}_{3}\right)$ $\delta 7.37(\mathrm{~d}, J=8.6 \mathrm{~Hz}, 2 \mathrm{H}), 7.13(\mathrm{dd}, J=7.9,1.5 \mathrm{~Hz}, 1 \mathrm{H}), 7.09-7.04(\mathrm{~m}, 1 \mathrm{H}), 6.96-6.87(\mathrm{~m}, 4 \mathrm{H}), 5.02-4.98(\mathrm{~m}, 1 \mathrm{H})$, 4.05-4.01 (m, 1H), $3.92(\mathrm{~s}, 3 \mathrm{H}), 3.81(\mathrm{~s}, 3 \mathrm{H}), 3.64-3.61(\mathrm{~m}, 1 \mathrm{H}), 3.49-3.43(\mathrm{~m}, 1 \mathrm{H}), 3.60-3.58(\mathrm{~m}, 1 \mathrm{H}), 2.68(\mathrm{dd}$, $J=8.3,5.2 \mathrm{~Hz}, 1 \mathrm{H}) ;{ }^{13} \mathbf{C}$ NMR (126 MHz, $\left.\mathbf{C D C l}_{3}\right) \delta 159.6,151.4,147.8,131.8,128.4,124.3,121.8,121.1,114.1$, 112.3, 89.6, 73.8, 61.1, 56.0, 55.4; HRMS (ESI) $m / z$ : $[\mathrm{M}+\mathrm{Na}]^{+}$Calcd for $\mathrm{C}_{17} \mathrm{H}_{20} \mathrm{NaO}_{5}$ 327.1203; Found 327.1203.

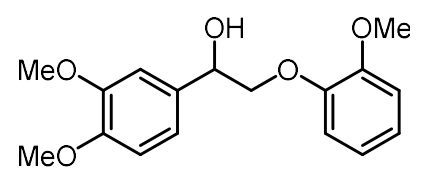

\section{1-(3,4-Dimethoxyphenyl)-2-(2-methoxyphenoxy)ethan-1-ol ( $\left.1 \mathrm{c}_{\text {red }}\right)$}

White solid; $\mathbf{R}_{\mathbf{f}}=0.3$ (hexanes/EtOAc 1:1); ${ }^{1} \mathbf{H}$ NMR (500 MHz, $\left.\mathbf{C D C l}_{3}\right) \delta$ 7.03-6.97 (m, 2H), 6.96-6.88 (m, 4H), $6.85(\mathrm{~d}, J=8.2 \mathrm{~Hz}, 1 \mathrm{H}), 5.05(\mathrm{dd}, J=9.3,2.8 \mathrm{~Hz}, 1 \mathrm{H}), 4.17(\mathrm{dd}, J=10.1,2.8 \mathrm{~Hz}, 1 \mathrm{H}), 3.97(\mathrm{t}, J=9.7 \mathrm{~Hz}, 1 \mathrm{H})$, 3.90 (s, 3H), 3.88 (s, 3H), 3.88 (s, 3H), 3.47 (br, 1H); ${ }^{13} \mathbf{C}$ NMR (126 MHz, CDCl $) \delta$ 150.1, 149.2, 148.8, 148.1, 132.3, 122.6, 121.2, 118.7, 115.9, 112.1, 111.1, 109.5, 76.3, 72.2, 56.0, 56.0, 55.9; HRMS (ESI) $\mathrm{m} / z$ : $[\mathrm{M}+\mathrm{Na}]^{+}$ Calcd for $\mathrm{C}_{17} \mathrm{H}_{20} \mathrm{NaO}_{5}$ 327.1203; Found 327.1205. 
<smiles>COc1ccc(C(O)C(CO)Oc2ccccc2OC)cc1OC</smiles>

1-(3,4-Dimethoxyphenyl)-2-(2-methoxyphenoxy)propane-1,3-diol (1d $\left.{ }_{\text {red }}\right)$

Major diastereomer (erythro): ${ }^{1} \mathbf{H}$ NMR (500 MHz, $\left.\mathbf{C D C l}_{3}\right): \delta 7.08-7.02(\mathrm{~m}, 1 \mathrm{H}), 6.99-6.87(\mathrm{~m}, 5 \mathrm{H}), 6.83(\mathrm{~d}$, $J=8.2 \mathrm{~Hz}, 1 \mathrm{H}), 4.97(\mathrm{~d}, J=4.6 \mathrm{~Hz}, 1 \mathrm{H}), 4.16(\mathrm{ddd}, J=5.8,4.6,3.3 \mathrm{~Hz}, 1 \mathrm{H}), 3.91(\mathrm{dd}, J=12.2,5.8 \mathrm{~Hz}, 1 \mathrm{H}), 3.87$ $(\mathrm{s}, 3 \mathrm{H}), 3.86(3 \mathrm{H}), 3.86(\mathrm{~s}, 3 \mathrm{H}), 3.66(\mathrm{dd}, J=12.2,3.3 \mathrm{~Hz}, 1 \mathrm{H}), 2.86(\mathrm{br}, 1 \mathrm{H}), 1.85$ (br, $1 \mathrm{H}) .{ }^{13} \mathbf{C}$ NMR (126 MHz, $\left.\mathbf{C D C l}_{3}\right): \delta 151.7,149.1,148.6,147.0,132.7,124.3,121.7,121.0,118.5,112.3,111.1,109.4,87.4,72.8,60.9,56.0$ (2C), 55.9. Minor diastereomer (threo): ${ }^{1} \mathbf{H}$ NMR (500 $\left.\mathbf{M H z}, \mathbf{C D C l}_{3}\right): \delta 7.12$ (dd, $\left.J=7.9,1.4 \mathrm{~Hz}, 1 \mathrm{H}\right), 7.08-7.02$ (m, 1H), 6.99-6.87 (m, 4H), $6.84(\mathrm{~d}, J=7.9 \mathrm{~Hz}, 1 \mathrm{H}), 4.98(\mathrm{~d}, J=7.6 \mathrm{~Hz}, 1 \mathrm{H}), 4.05-4.01(\mathrm{~m}, 1 \mathrm{H}), 3.90(\mathrm{~s}, 3 \mathrm{H})$, $3.87(\mathrm{~s}, 6 \mathrm{H}), 3.63(\mathrm{dd}, J=12.5,3.3 \mathrm{~Hz}, 1 \mathrm{H}), 3.48(\mathrm{dd}, J=12.5,3.7 \mathrm{~Hz}, 1 \mathrm{H}), 2.86(\mathrm{br}, 1 \mathrm{H}), 1.85$ (br, $1 \mathrm{H}) .{ }^{13} \mathbf{C}$ NMR (126 MHz, $\left.\mathbf{C D C l}_{3}\right): \delta 151.4,149.2,149.0,147.7,132.2,124.3,121.8,121.1,119.7,112.3,111.1,110.0,89.5,74.0$, 61.1, 56.0 (2C), 55.9. HRMS (ESI) $m / z:[\mathrm{M}+\mathrm{Na}]^{+}$Calcd for $\mathrm{C}_{18} \mathrm{H}_{22} \mathrm{NaO}_{6}$ 357.1309; Found: 357.1313 .

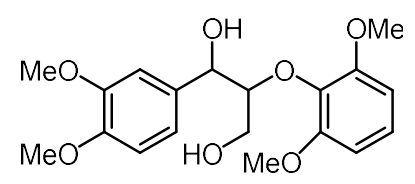

2-(2,6-Dimethoxyphenoxy)-1-(3,4-dimethoxyphenyl)propane-1,3-diol (1f $\left.\mathrm{fed}_{\text {red }}\right)$

White solid; $\mathbf{R}_{\mathbf{f}}=0.17$ (hexanes/EtOAc 1:1); Major diastereomer (erythro): ${ }^{1} \mathbf{H}$ NMR (500 MHz, $\left.\mathbf{C D C l}_{3}\right) \delta 7.11-$ $7.04(\mathrm{~m}, 1 \mathrm{H}), 6.95(\mathrm{~s}, 1 \mathrm{H}), 6.87-6.80(\mathrm{~m}, 2 \mathrm{H}), 6.67-6.62(\mathrm{~m}, 2 \mathrm{H}), 5.03(\mathrm{~d}, J=3.2 \mathrm{~Hz}, 1 \mathrm{H}), 4.18-4.14(\mathrm{~m}, 1 \mathrm{H}), 4.13$ (br s, 1H), 3.89 (s, 9H), 3.86 (s, 3H), 3.52-3.46 (m, 1H), 3.17-3.09 (m, 1H); ${ }^{13} \mathbf{C}$ NMR (126 MHz, CDCl $) \delta 153.6$, 149.1, 148.3, 135.1, 132.1, 124.6, 118.2, 111.1, 109.1, 105.4, 87.1, 72.5, 60.7, 56.2, 56.0; Minor diastereomer (threo): ${ }^{1} \mathbf{H}$ NMR (500 MHz, $\left.\mathbf{C D C l}_{3}\right) \delta$ 7.11-7.04 (m, 1H), $7.01(\mathrm{~s}, 1 \mathrm{H}), 6.87-6.80(\mathrm{~m}, 2 \mathrm{H}), 6.67-6.62(\mathrm{~m}, 2 \mathrm{H})$, $5.06(\mathrm{~d}, J=8.9 \mathrm{~Hz}, 1 \mathrm{H}), 4.35$ (br s, 1H), $3.91(\mathrm{~s}, 6 \mathrm{H}), 3.89$ (s, 3H), 3.87 (s, 3H), 3.60-3.55 (m, 1H), 3.37-3.28 (m, 2H); ${ }^{13} \mathbf{C}$ NMR (126 MHz, $\left.\mathbf{C D C l}_{3}\right) \delta 153.3,149.0,148.8,135.4,132.7,124.6,119.9,111.1,110.4,105.4,89.1$, 74.1, 60.5, 56.2, 56.0; HRMS (ESI) $m / z$ : [M + Na $]^{+}$Calcd for $\mathrm{C}_{19} \mathrm{H}_{24} \mathrm{NaO}_{7}$ 387.1414; Found 387.1417.

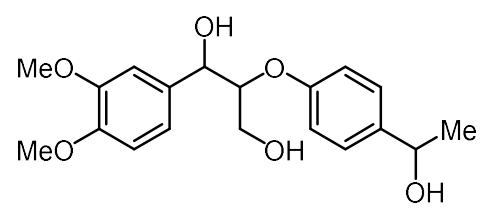

\section{1-(3,4-Dimethoxyphenyl)-2-(4-(1-hydroxyethyl)phenoxy)propane-1,3-diol ( $\left.1 \mathrm{~h}_{\text {red }}\right)$}

Pale yellow solid; $\mathbf{R}_{\mathbf{f}}=0.24$ (hexanes/EtOAc/EtOH 70:23:7); Major diastereomers (erythro): ${ }^{1} \mathbf{H}$ NMR (500 MHz, $\left.\mathbf{C D C l}_{3}\right) \delta 7.31(\mathrm{~d}, J=8.5 \mathrm{~Hz}, 2 \mathrm{H}), 7.01-6.95(\mathrm{~m}, 3 \mathrm{H}), 6.90-6.82(\mathrm{~m}, 2 \mathrm{H}), 5.00(\mathrm{dd}, J=6.8,2.6 \mathrm{~Hz}, 1 \mathrm{H})$, $4.90-4.82(\mathrm{~m}, 1 \mathrm{H}), 4.43-4.37(\mathrm{~m}, 1 \mathrm{H}), 3.88(\mathrm{~s}, 6 \mathrm{H}), 3.85-3.79(\mathrm{~m}, 1 \mathrm{H}), 3.63-3.56(\mathrm{~m}, 1 \mathrm{H}), 2.74(\mathrm{~d}, J=2.8 \mathrm{~Hz}$, 
1H), 1.75-1.68 (m, 2H), 1.50-1.45 (m, 3H); ${ }^{13} \mathbf{C}$ NMR (126 MHz, CDCl $) \delta 157.3,148.7,148.5,139.0,132.6$, 126.7, 119.1, 116.2, 110.9, 110.0, 82.6, 73.0, 69.5, 60.8, 55.8, 24.9; Minor diastereomers (threo): ${ }^{1}$ H NMR (500 MHz, $\left.\mathbf{C D C l}_{3}\right) \delta 7.27(\mathrm{~d}, J=8.5 \mathrm{~Hz}, 2 \mathrm{H}), 7.01-6.95(\mathrm{~m}, 4 \mathrm{H}), 6.90-6.82(\mathrm{~m}, 1 \mathrm{H}), 5.05-5.02(\mathrm{~m}, 1 \mathrm{H}), 4.90-4.82(\mathrm{~m}$, $1 \mathrm{H}), 4.43-4.37(\mathrm{~m}, 1 \mathrm{H}), 3.98-3.91(\mathrm{~m}, 2 \mathrm{H}), 3.87(\mathrm{~s}, 6 \mathrm{H}), 2.63(\mathrm{~d}, J=3.2 \mathrm{~Hz}, 1 \mathrm{H}), 2.13(\mathrm{t}, J=6.6 \mathrm{~Hz}, 1 \mathrm{H}), 1.75-$ 1.68 (m, 1H), 1.50-1.45 (m, 3H); ${ }^{13} \mathbf{C}$ NMR (126 MHz, $\left.\mathbf{C D C l}_{3}\right) \delta 157.0,148.7,148.4,138.9,133.3,126.6,118.8$, 116.3, 110.9, 109.8, 81.9, 73.6, 69.5, 61.3, 55.8, 24.9; HRMS (ESI) $m / z$ : $[\mathrm{M}+\mathrm{Na}]^{+}$Calcd for $\mathrm{C}_{19} \mathrm{H}_{24} \mathrm{NaO}_{6}$ 371.1465; Found 371.1456. 


\section{Photon flux determination}

Photon flux of violet LED strips and Kessil PR160-390nm were measured according to reported methods ${ }^{[\mathrm{S} 4]}$.

Solution A

$\mathrm{H}_{2} \mathrm{SO}_{4}(0.05 \mathrm{M})$ in deionized water

Solution B

$\mathrm{K}_{3} \mathrm{FeC}_{2} \mathrm{O}_{4}(0.15 \mathrm{M})$ and $\mathrm{H}_{2} \mathrm{SO}_{4}(0.05 \mathrm{M})$ in deionized water

Solution C (developer solution)

$\mathrm{NaOAc}(2.75 \mathrm{M}), 1,10$-phenantroline $(0.05 \mathrm{M})$ and $\mathrm{H}_{2} \mathrm{SO}_{4}(0.5 \mathrm{M})$ in deionized water

\section{Experiment:}

A $1 \mathrm{~cm}$ x $1 \mathrm{~cm}$ quartz cuvette was charged with $3 \mathrm{~mL}$ of solution $\mathrm{B}$. Two sides of the cuvette were coated with black electrical tape to ensure a minimum pathway of the light of $1 \mathrm{~cm}$. To stir the ferrioxolate, the solution was continually sparged with $\mathrm{N}_{2}$. While stirring, the solution was irradiated with LED at room temperature. $10 \mu \mathrm{L}$ aliquots of the solution were taken at different time points. This aliquot was immediately added to $5 \mathrm{~mL}$ of solution $\mathrm{C}$ (developer solution) and the flask was quickly wrapped in aluminum foil. Concurrently, a "blank" sample was prepared by diluting $10 \mathrm{uL}$ of the solution A into $5 \mathrm{~mL}$ of solution $\mathrm{C}$ (developer solution). The solutions were left in the dark for 1 hour, becoming bright red. The solutions were transferred to a cuvette and the absorbance spectrum of the $\mathrm{Fe}(\text { phen })_{3}{ }^{2+}$ complex was obtained. The absorbance at $510 \mathrm{~nm}$ was measured for every sample.

Results:
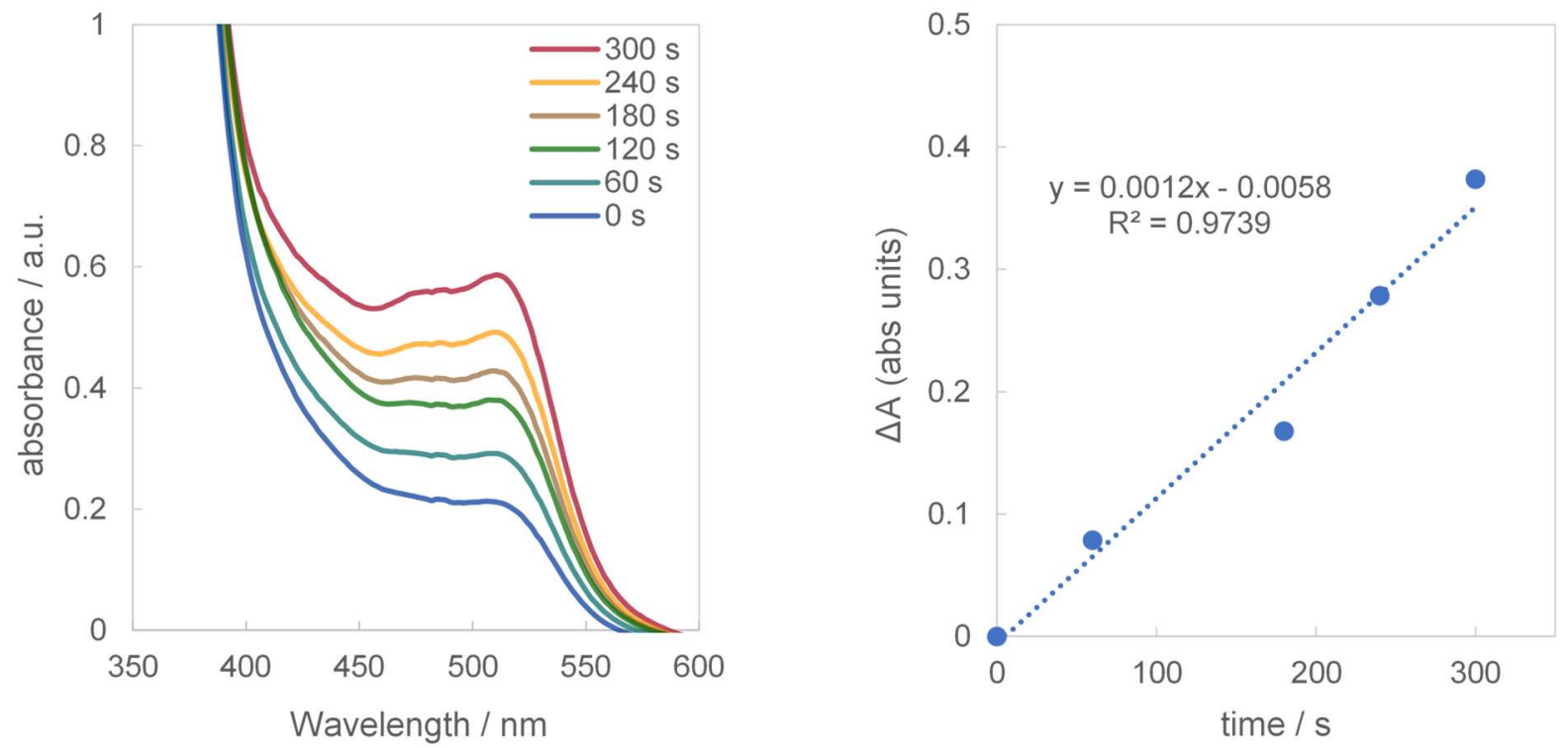

Figure S4. Summary of photon flux determination (violet LED strips $410 \mathrm{~nm}$ ) 

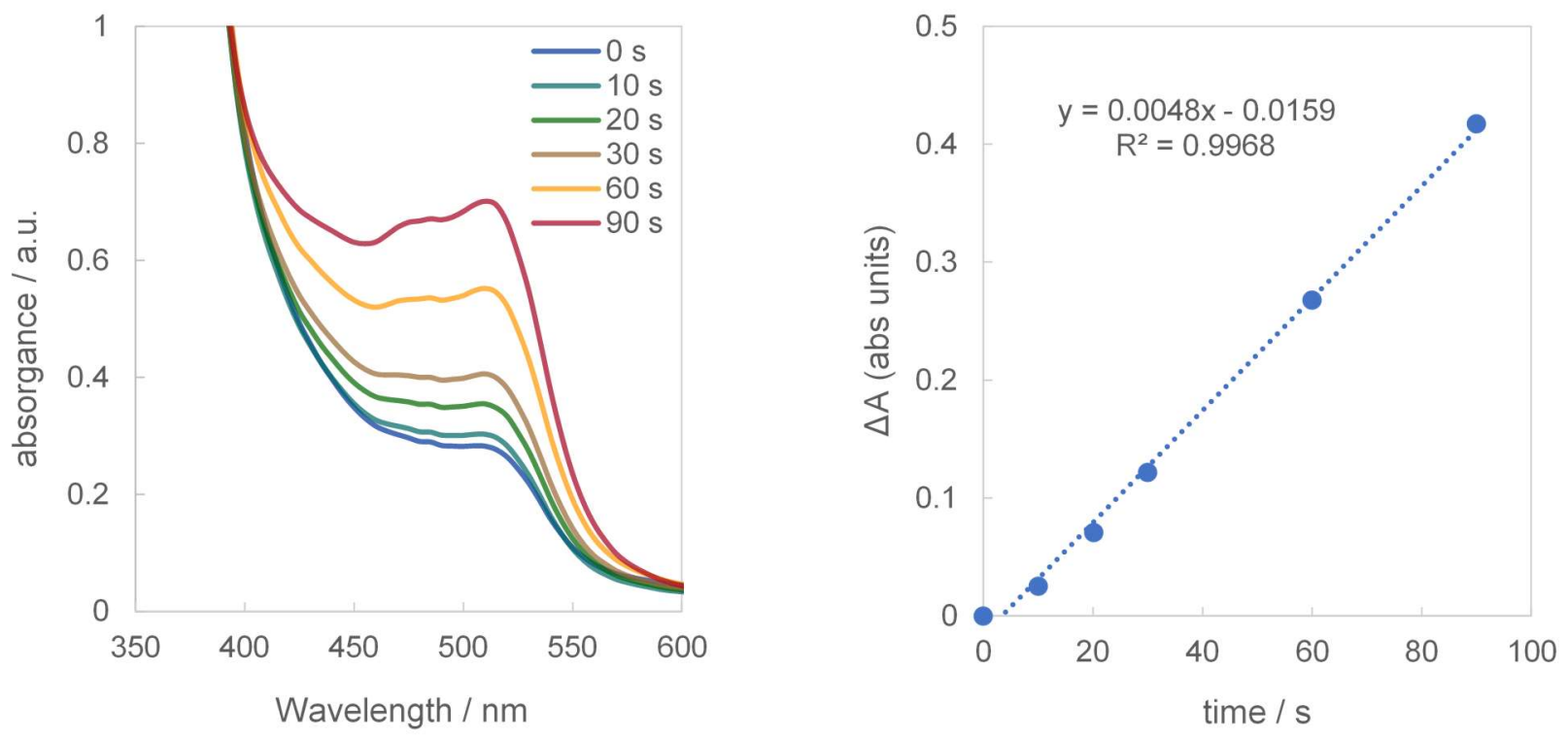

Figure S5. Summary of photon flux determination (Kessil LED PR160-390 nm)

The photon flux was determined by the following equation.

$$
\text { photon flux }=\frac{\Delta A_{\text {wavelengt }} \times V_{1} \times V_{3}}{\varepsilon_{510 \mathrm{~nm}} \times l \times V_{2}} \times \frac{1}{\phi_{\text {wavelength }} \times t \times F} \times \frac{1}{S}
$$

photon flux $=$ the number of photons per second per unit area

$\Delta A_{\text {wavelength }}=$ difference in absorbance at $510 \mathrm{~nm}$ between sample and 'blank'

$I=$ path length of cuvette $(1 \mathrm{~cm})$

$\varepsilon_{510 \mathrm{~nm}}=$ extinction coefficient of $\mathrm{Fe}(\mathrm{phen})_{3}$ complex at $510\left(11,100 \mathrm{M}^{-1} \mathrm{~cm}^{-1}\right)$

$V_{1}=$ total volume of irradiated solution $(3 \mathrm{~mL})$

$V_{2}=$ volume of aliquot taken from $\mathrm{V}_{1}(10 \mathrm{uL})$

$V_{3}=$ the volume that $\mathrm{V}_{2}$ is diluted into $(5 \mathrm{~mL})$

$\emptyset_{\text {wavelength }}=$ quantum efficiency of ferrioxalate $\left(\sim 1.11\right.$ at $410 \mathrm{~nm}, \sim 1.12$ at $390 \mathrm{~nm}$, estimated from reported data $\left.{ }^{[\mathrm{S} 5]}\right)$

$t=$ time of irradiation (s)

$F=$ mean fraction of light absorbed by the ferrioxalate solution $(\sim 1)$

$S=$ surface area $\left(3 \mathrm{~cm}^{2}\right)$

The calculation results are shown below.

$$
\begin{aligned}
& \text { photon flux (violet LED strips, } 410 \mathrm{~nm})=0.05 \times 10^{-6}\left(\mathrm{~mol} \mathrm{~s}^{-1} \mathrm{~cm}^{-2}\right) \\
& \text { photon flux (Kessil LED, } 390 \mathrm{~nm}, 25 \% \text { intensity) }=0.20 \times 10^{-6}\left(\mathrm{~mol} \mathrm{~s}^{-1} \mathrm{~cm}^{-2}\right)
\end{aligned}
$$

Conclusions:

The photon flux of Kessil LED $390 \mathrm{~nm}$ (100\% intensity) is 16 times larger than that of violet LED strips. 


\section{Proposed mechanism of PhPTH-catalyzed photochemical reductive cleavage}

Mechanism is proposed based on ref S7-S9.

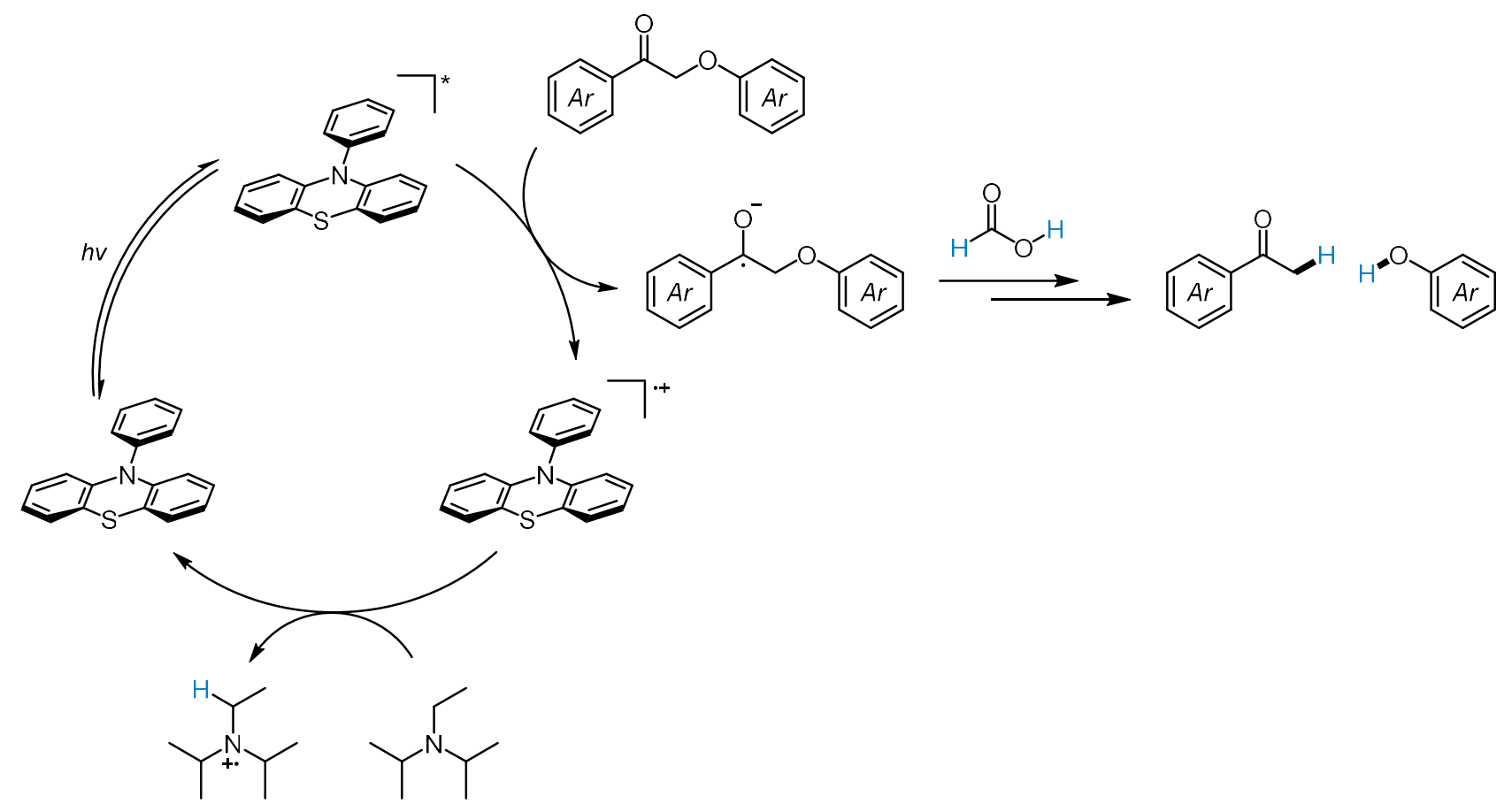

Figure S6. Proposed mechanism 


\section{References}

[S1] M. D. Kärkäs, I. Bosque, B. S. Matsuura, C. R. J. Stephenson, Org. Lett. 2016, 18, 5166-5169.

[S2] G. Magallanes, M. D. Kärkäs, I. Bosque, S. Lee, S. Maldonado, C. R. J. Stephenson, ACS Catal. 2019, 9, 22522260.

[S3] N. J. Treat, H. Sprafke, J. W. Kramer, P. G. Clark, B. E. Barton, J. Read de Alaniz, B. P. Fors, C. J. Hawker, J. Am. Chem. Soc. 2014, 136, 16096-16101.

[S4] H. G. Yayla, F. Peng, I. K. Mangion, M. McLaughlin, L. Campeau, I. W. Davies, D. A. DiRocco, R. R. Knowles, Chemical Science, 2016, 7, 2066-2073.

[S5] C. G. Hatchard, and C. A. Parker, Proceedings of the Royal Society of London. Series A, Mathematical and Physical Sciences, 1956, 235, 518-536.

[S6] Bosque, I.; Magallanes, G.; Rigoulet, M.; Kärkäs, M. D.; Stephenson, C. R. J. Redox Catalysis Facilitates Lignin Depolymerization. ACS Cent. Sci. 2017, 3, 621.

[S7] Narayanam, J. M. R.; Tucker, J. W.; Stephenson, C. R. J. Electron-Transfer Photoredox Catalysis: Development of a Tin-Free Reductive Dehalogenation Reaction. J. Am. Chem. Soc. 2009, 131, 8756-8757.

[S8] Treat, N. J.; Sprafke, H.; Kramer, J. W.; Clark, P. G.; Barton, B. E.; Alaniz, J. R.; Fors, B. P.; Hawker, C. J. Metal-Free Atom Transfer Radical Polymerization. J. Am. Chem. Soc. 2014, 136, 16096.

[S9] Nguyen, J. D.; Matsuura, B. S.; Stephenson, C. R. J. A Photochemical Strategy for Lignin Degradation at Room Temperature. J. Am. Chem. Soc. 2014, 136, 1218. 
12. NMR spectra
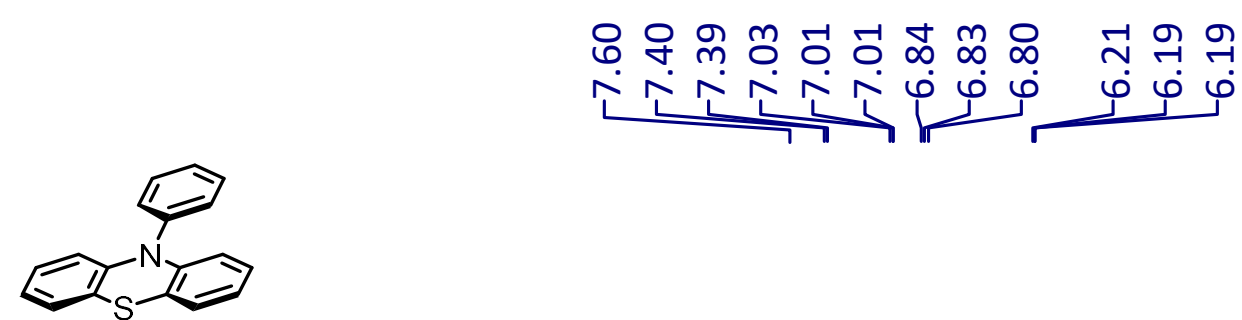

PhPTH

${ }^{1} \mathrm{H} \mathrm{NMR}\left(500 \mathrm{MHz}, \mathrm{CDCl}_{3}\right)$

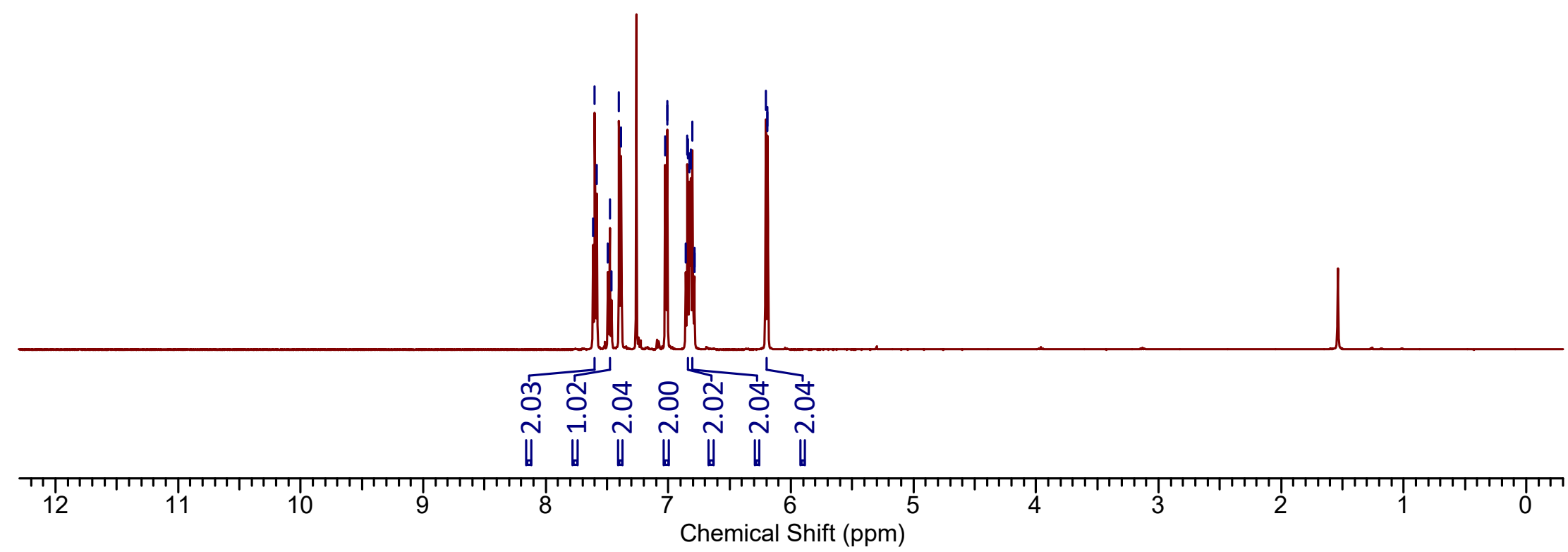




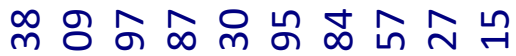

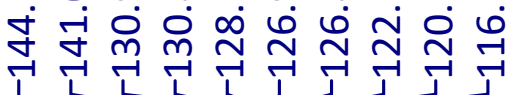

L

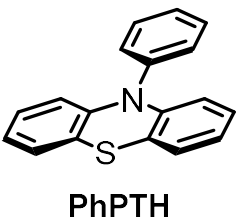

${ }^{13} \mathrm{C}$ NMR $\left(126 \mathrm{MHz}, \mathrm{CDCl}_{3}\right)$

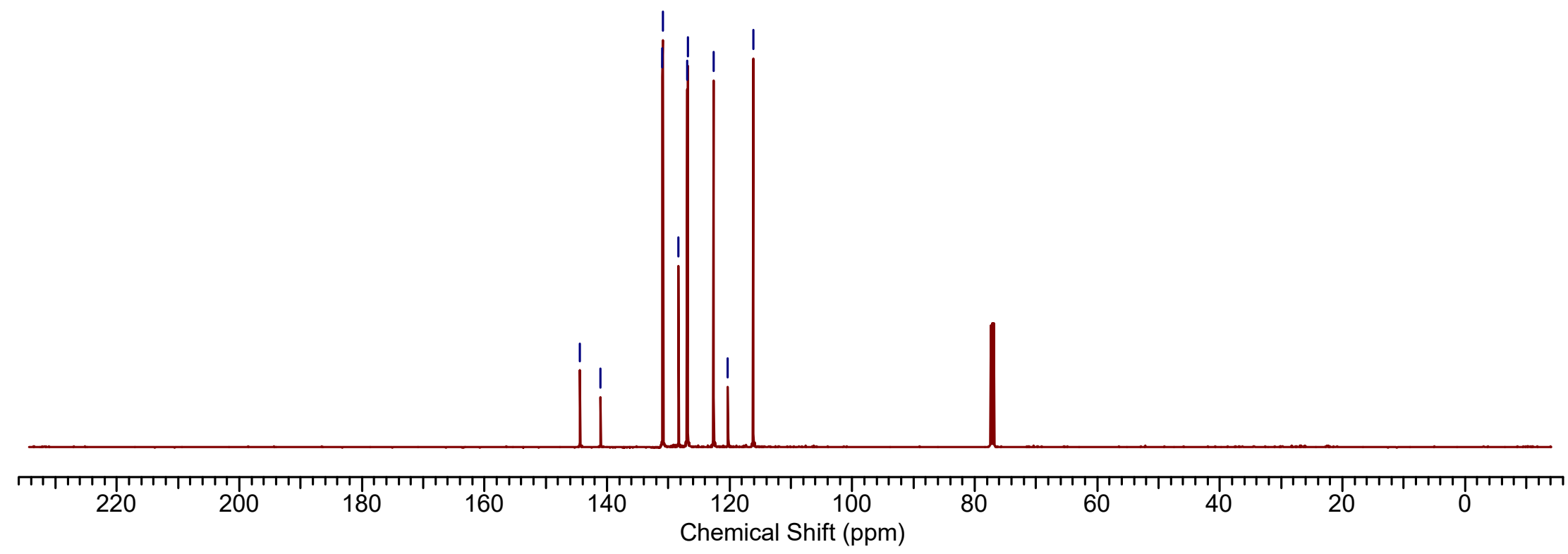



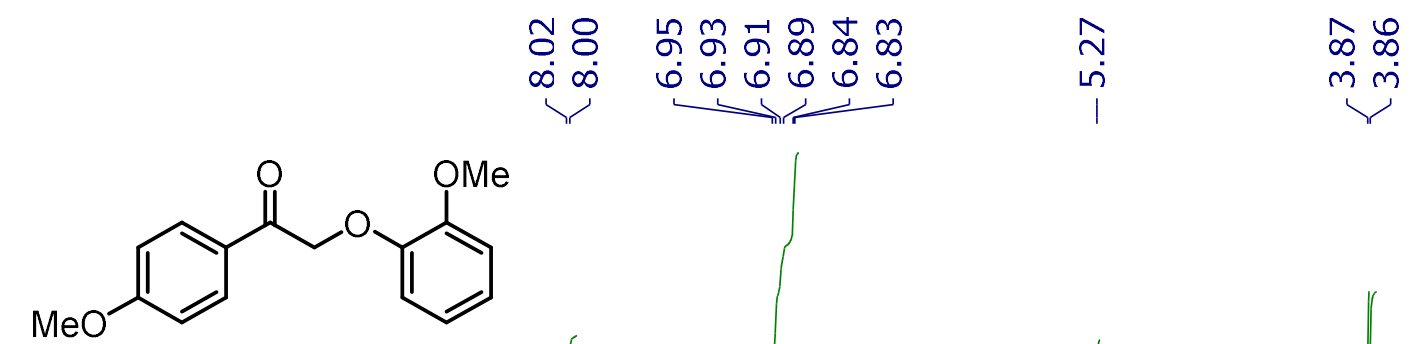

$1 a$

${ }^{1} \mathrm{H}$ NMR $\left(500 \mathrm{MHz}, \mathrm{CDCl}_{3}\right)$

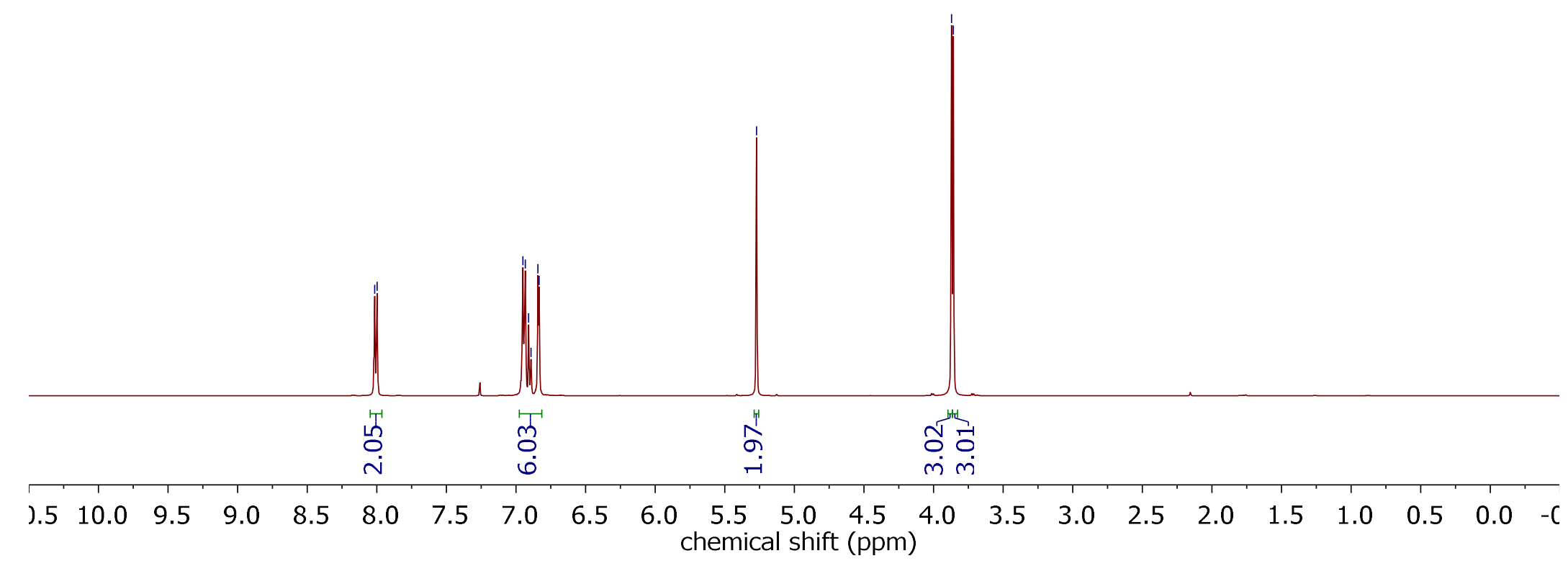



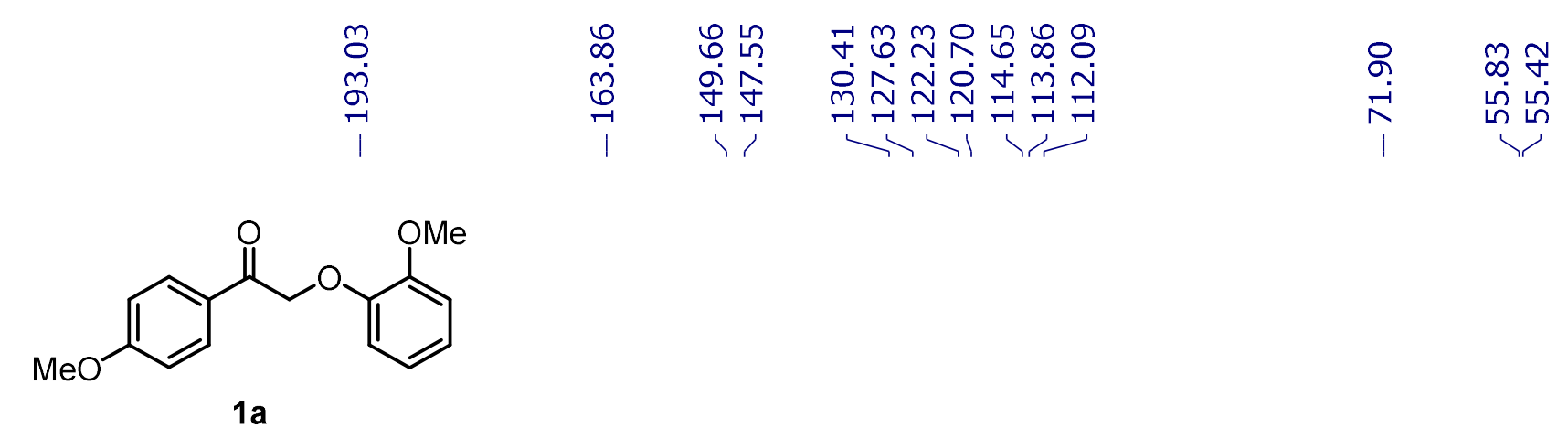

$\left.{ }^{13} \mathrm{C} \mathrm{NMR} \mathrm{(126} \mathrm{MHz,} \mathrm{CDCl}_{3}\right)$
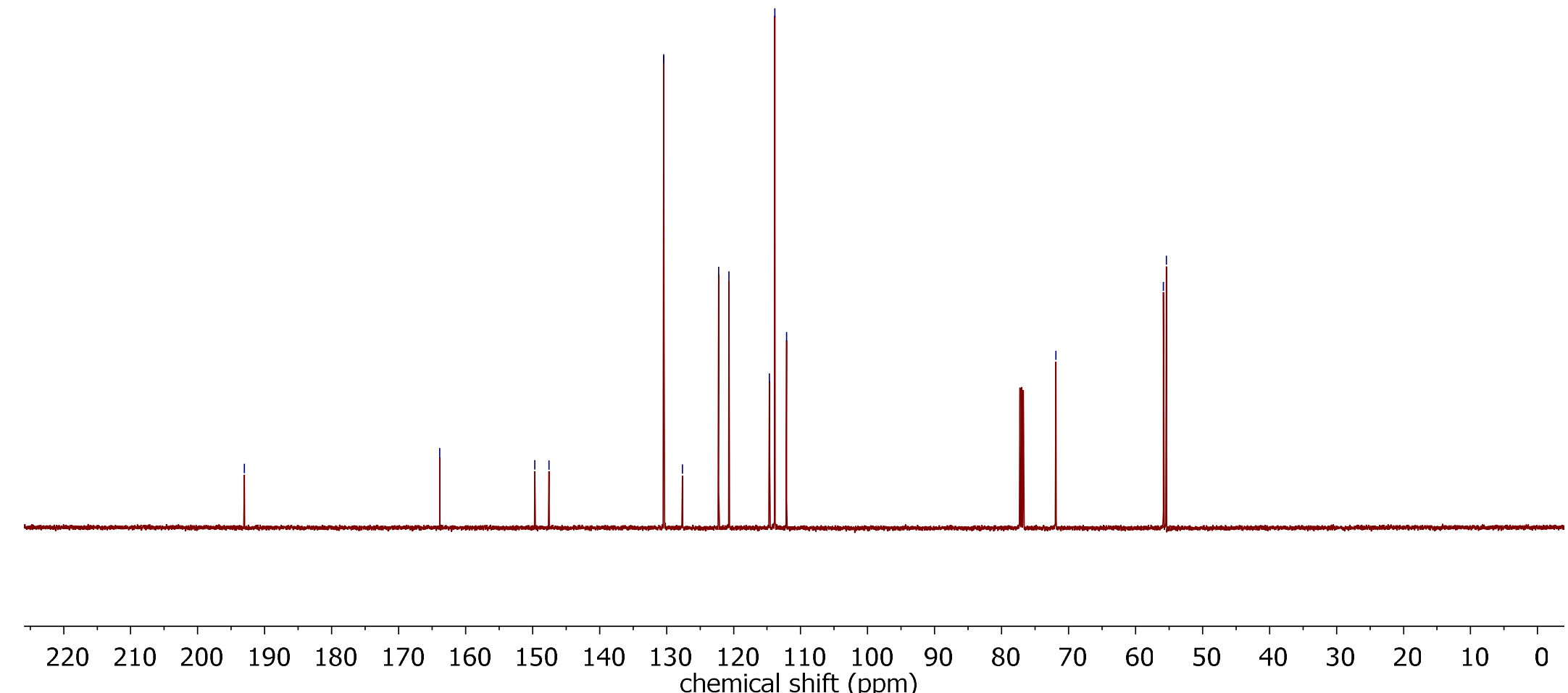


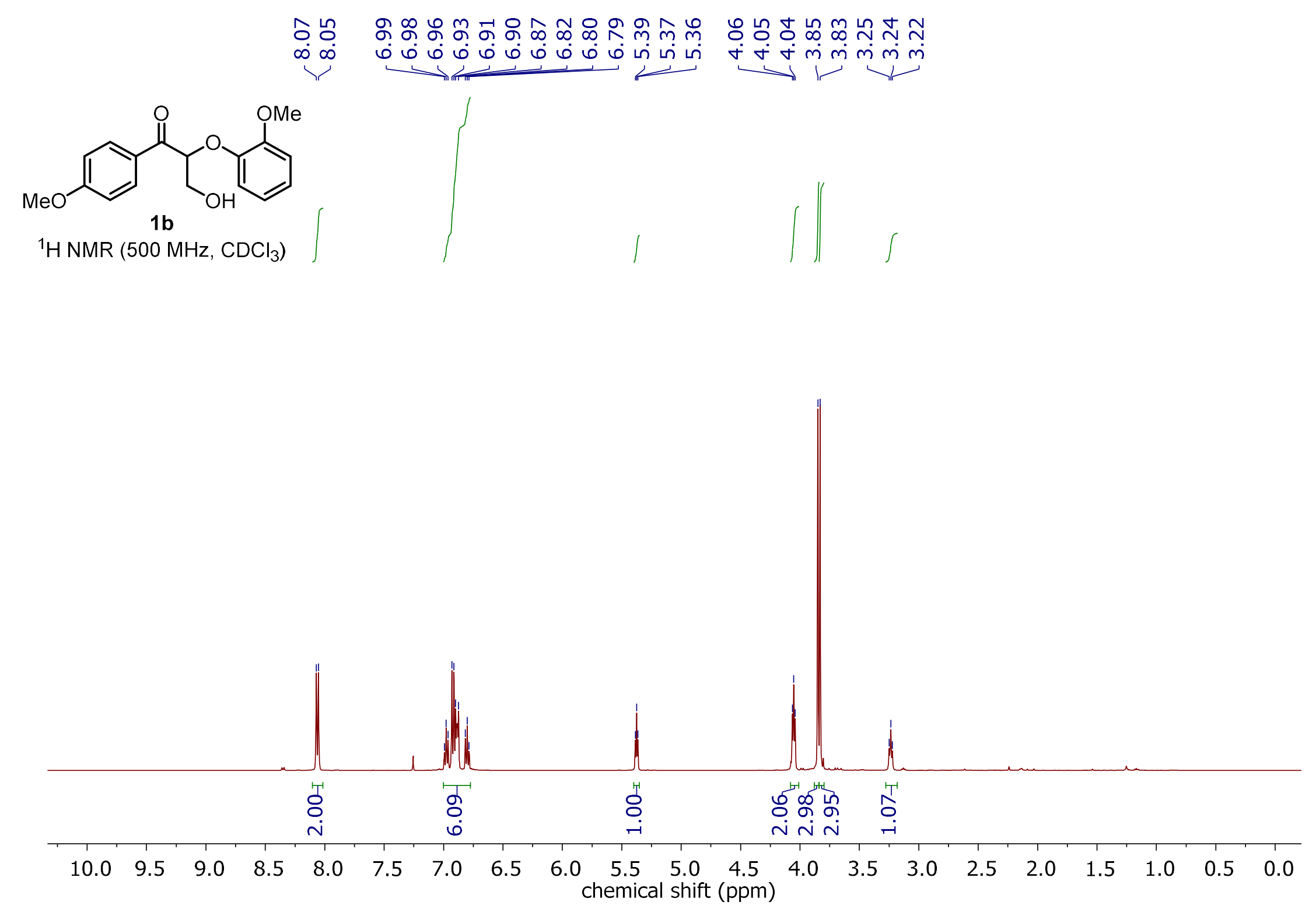



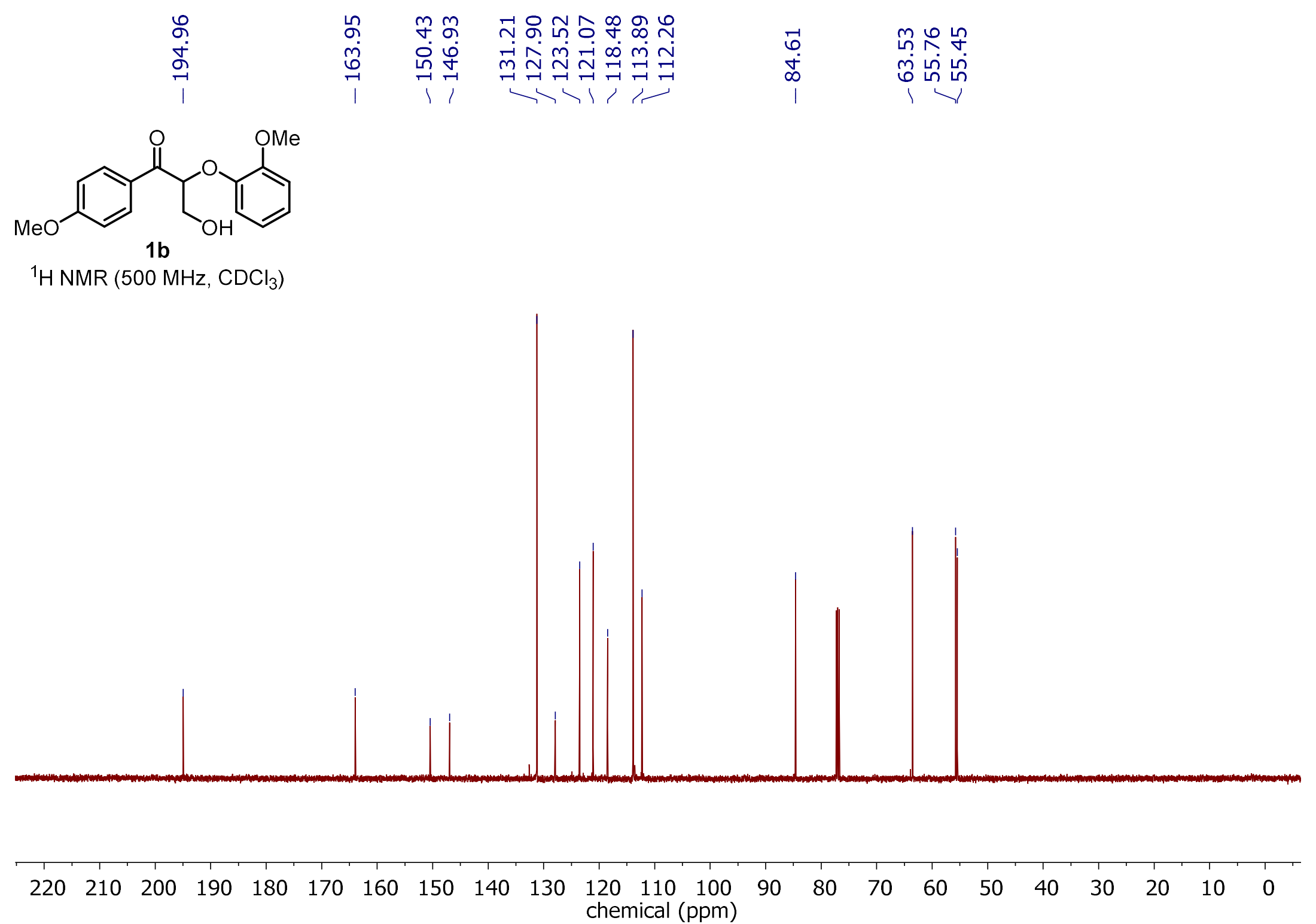


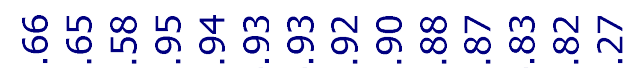

ה
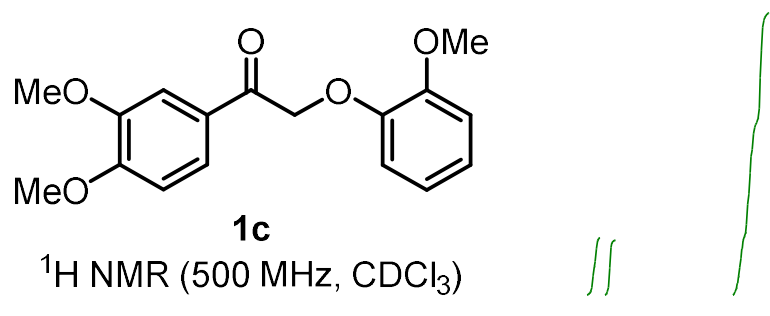

11 r

रू केष

$m m$

$\checkmark$

${ }^{1} \mathrm{H} \mathrm{NMR}\left(500 \mathrm{MHz}, \mathrm{CDCl}_{3}\right)$
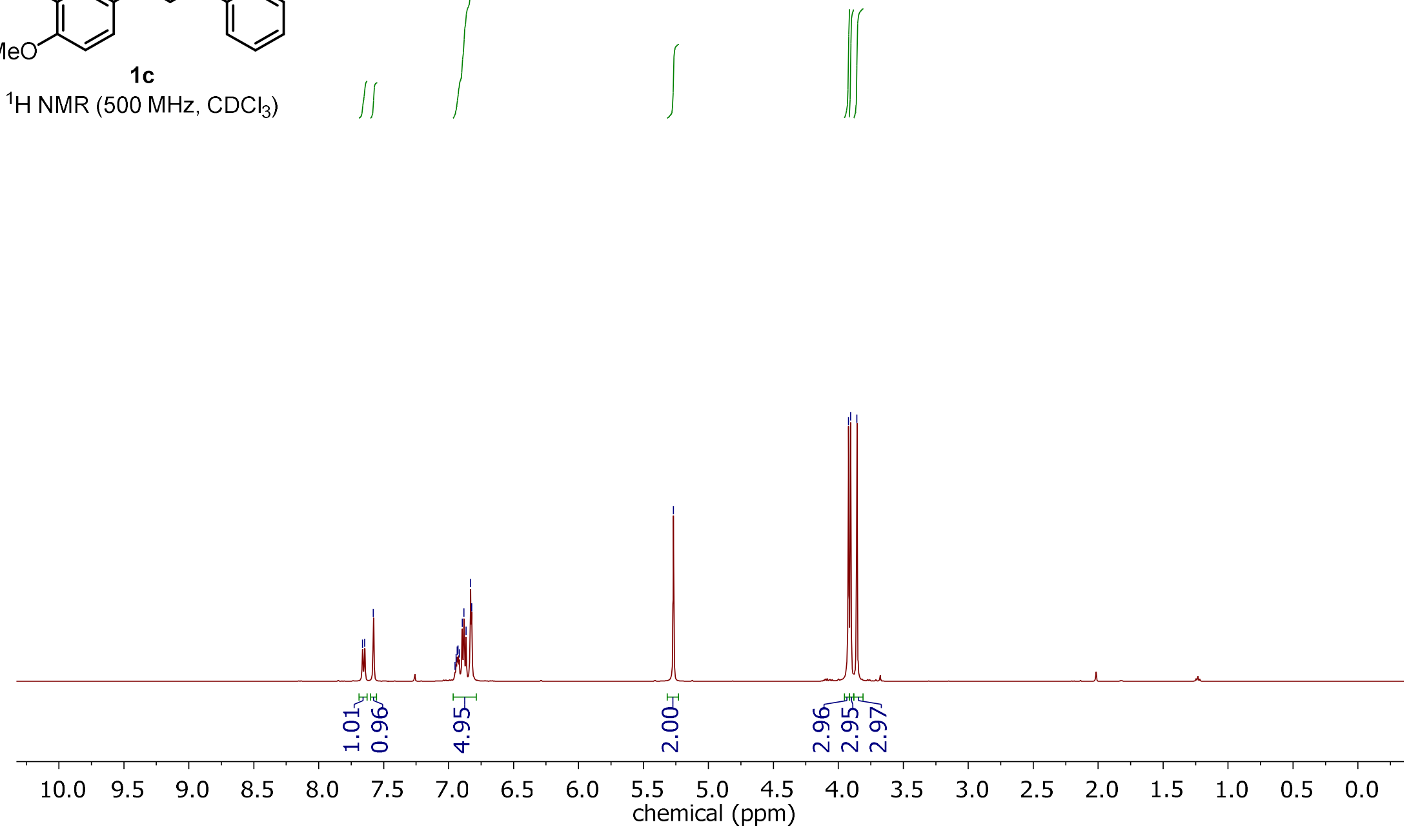
$\stackrel{\sim}{-}$
$\stackrel{2}{2}$
$\stackrel{-}{-1}$

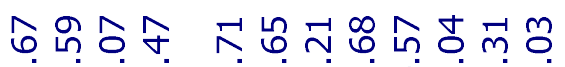

M) $\quad$ Mُ

$\infty \quad \infty \hat{\infty} \hat{\imath}$

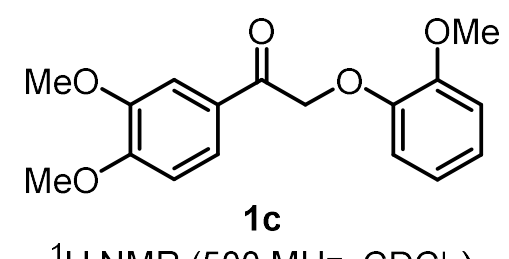

${ }^{1} \mathrm{H}$ NMR $\left(500 \mathrm{MHz}, \mathrm{CDCl}_{3}\right)$

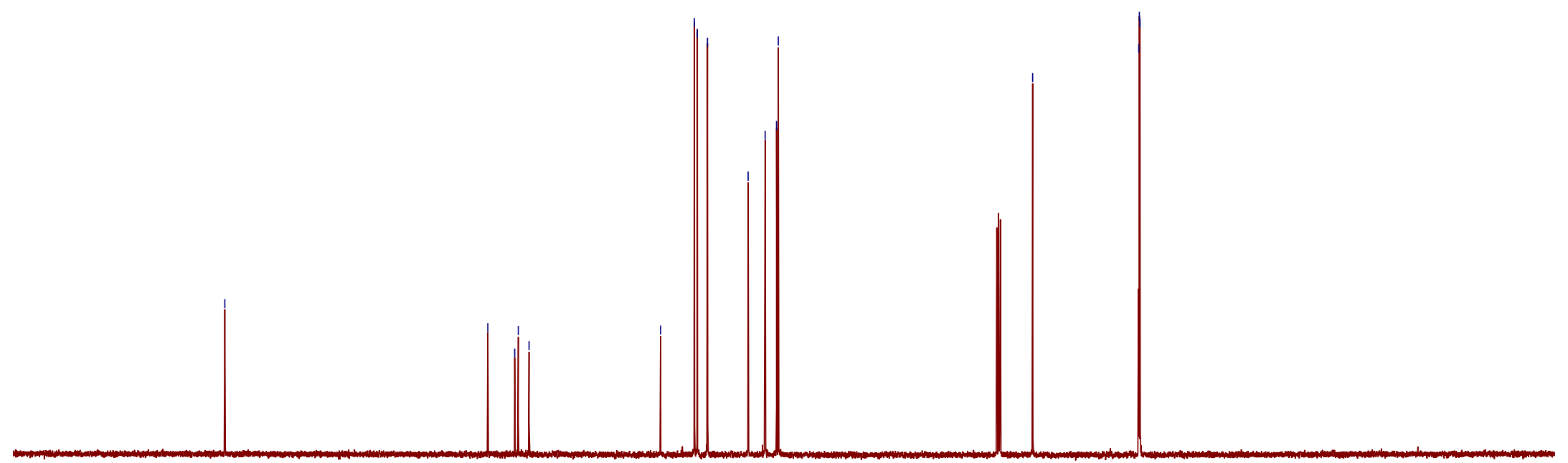

220

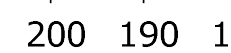

170160

$\begin{array}{lllllll}150 & 140 & 130 & 120 & 110 & 100 & 90\end{array}$

chemical shift (ppm) 


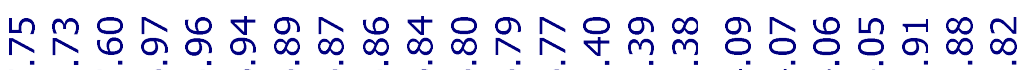
NNN

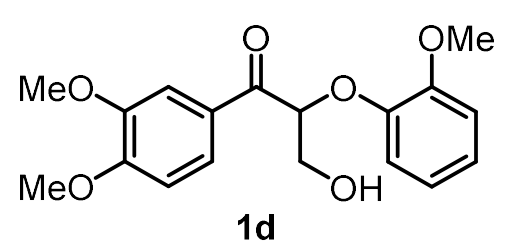

${ }^{1} \mathrm{H} \mathrm{NMR}\left(500 \mathrm{MHz}, \mathrm{CDCl}_{3}\right)$
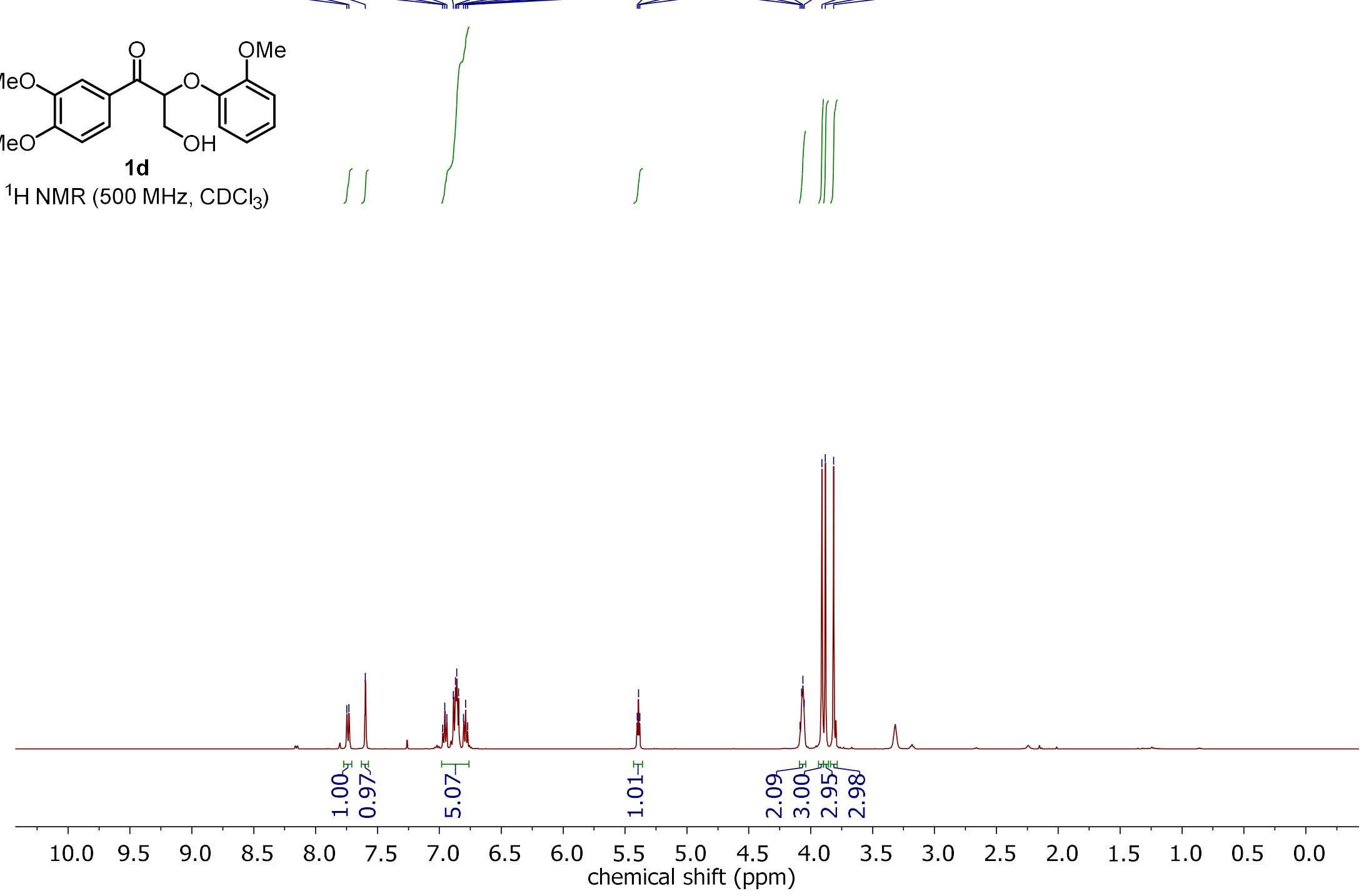


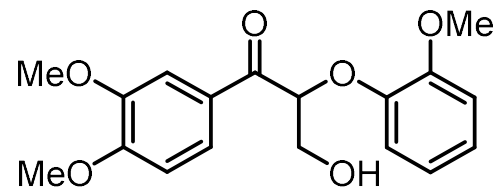

1d

${ }^{1} \mathrm{H}$ NMR $\left(500 \mathrm{MHz}, \mathrm{CDCl}_{3}\right)$
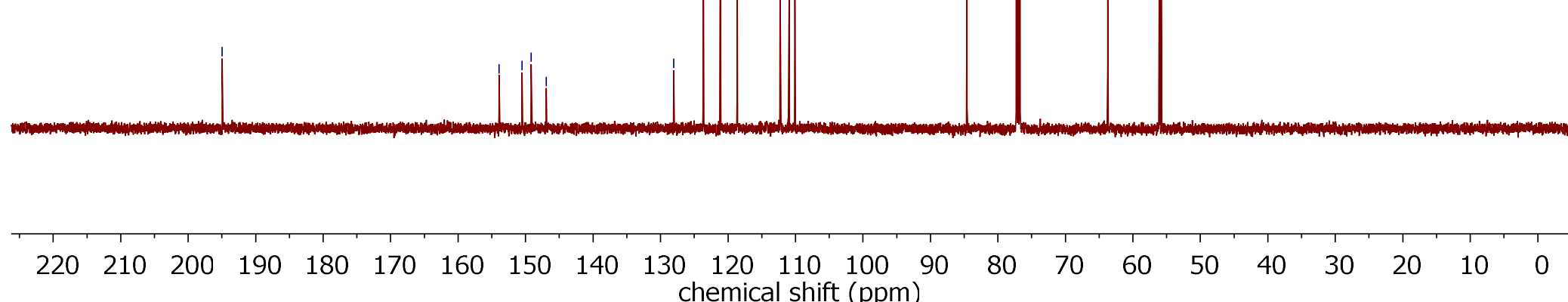


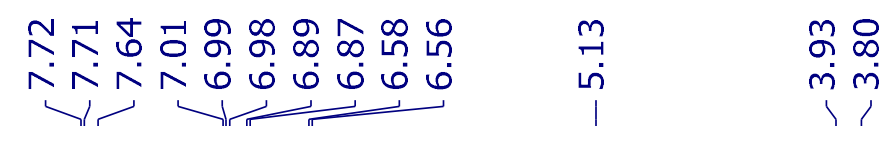

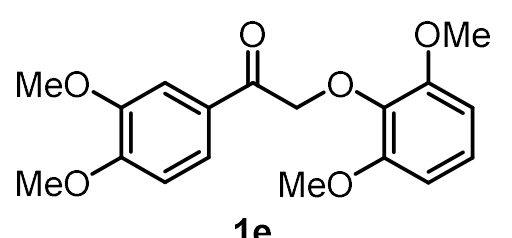

${ }^{1} \mathrm{H} \mathrm{NMR}\left(500 \mathrm{MHz}, \mathrm{CDCl}_{3}\right)$

NヘN

mi
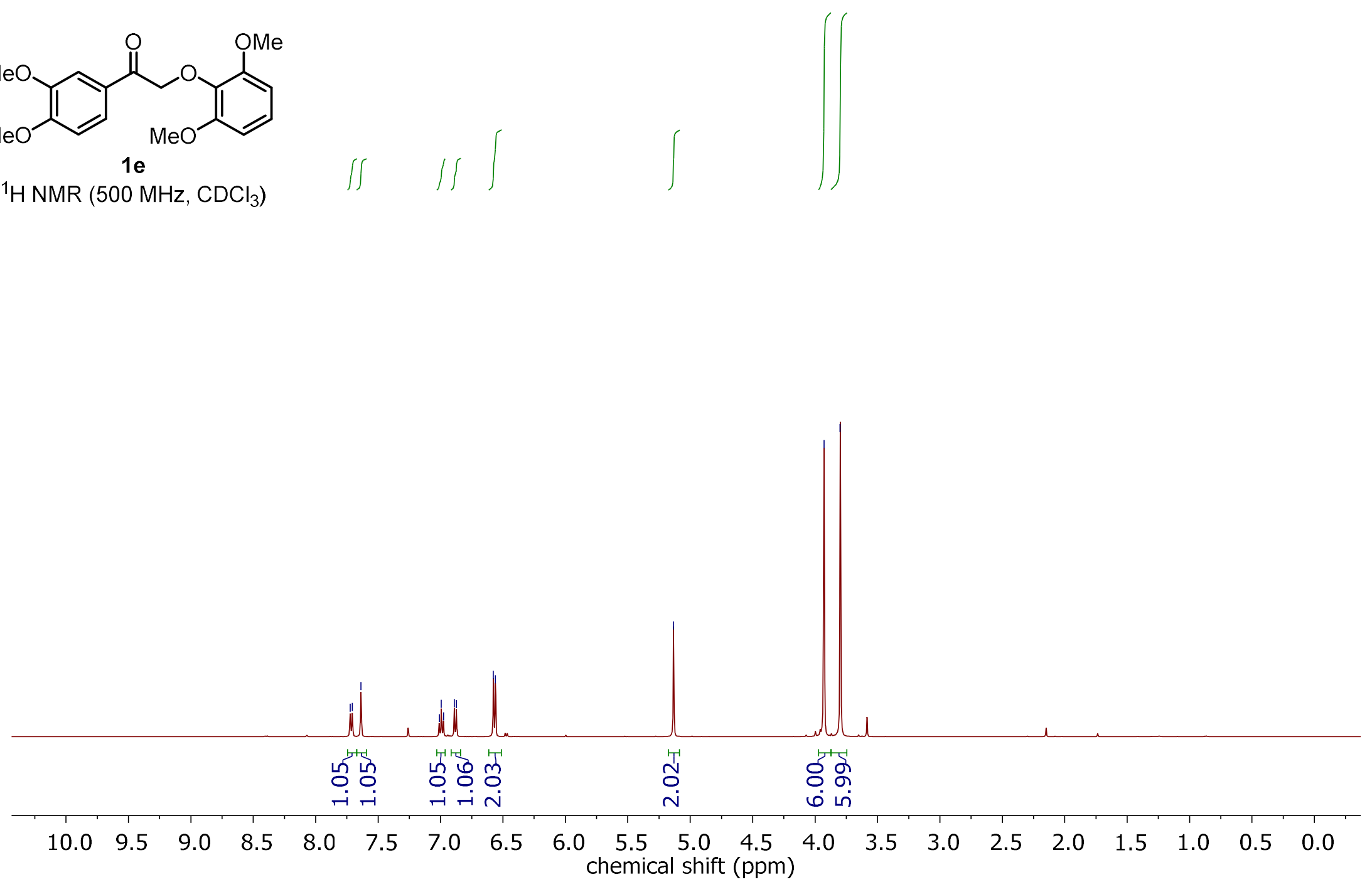

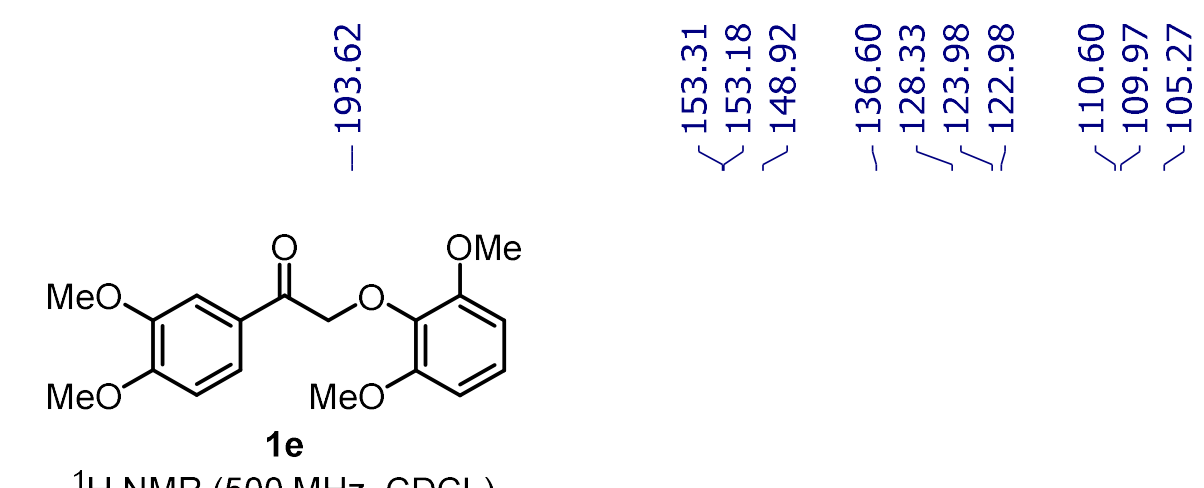

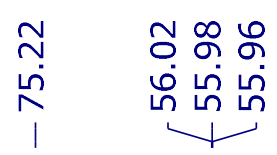

${ }^{1} \mathrm{H}$ NMR $\left(500 \mathrm{MHz}, \mathrm{CDCl}_{3}\right)$

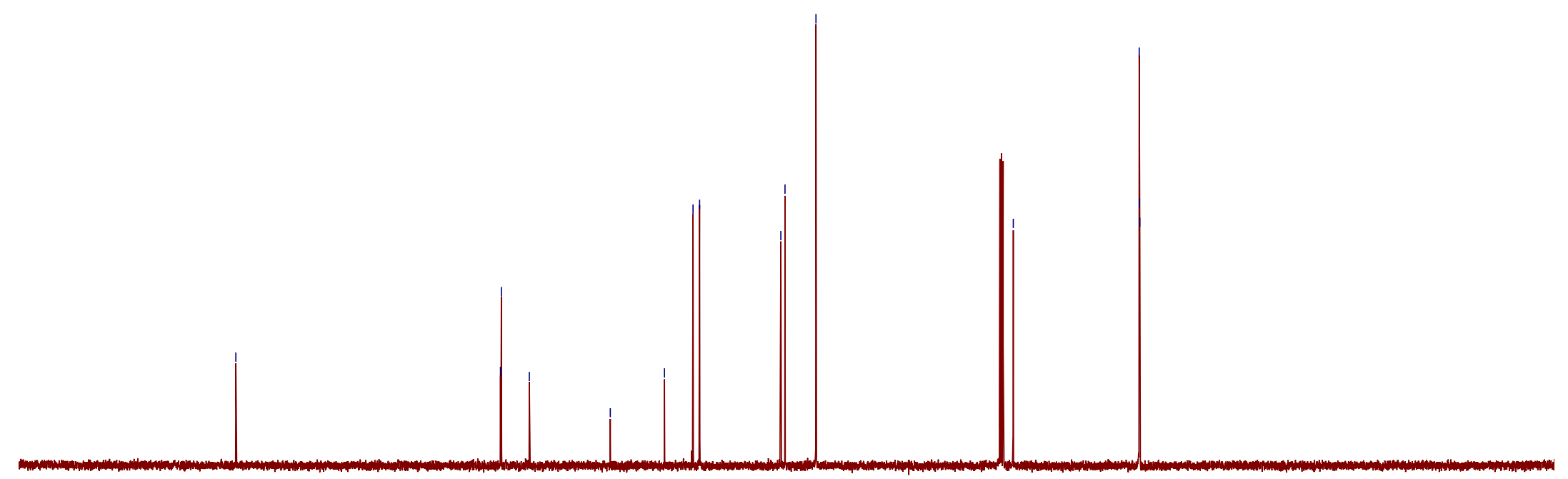

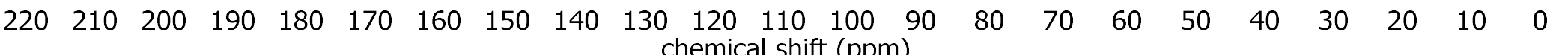




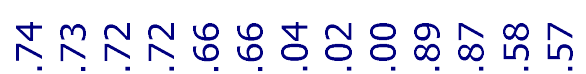

ヘNヘNヘNヘNヘ

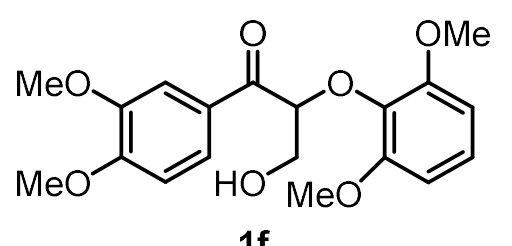

$1 \mathrm{f}$

${ }^{1} \mathrm{H} \mathrm{NMR}\left(500 \mathrm{MHz}, \mathrm{CDCl}_{3}\right)$

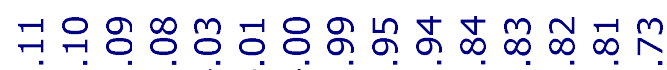

ம் ம் ம் ن

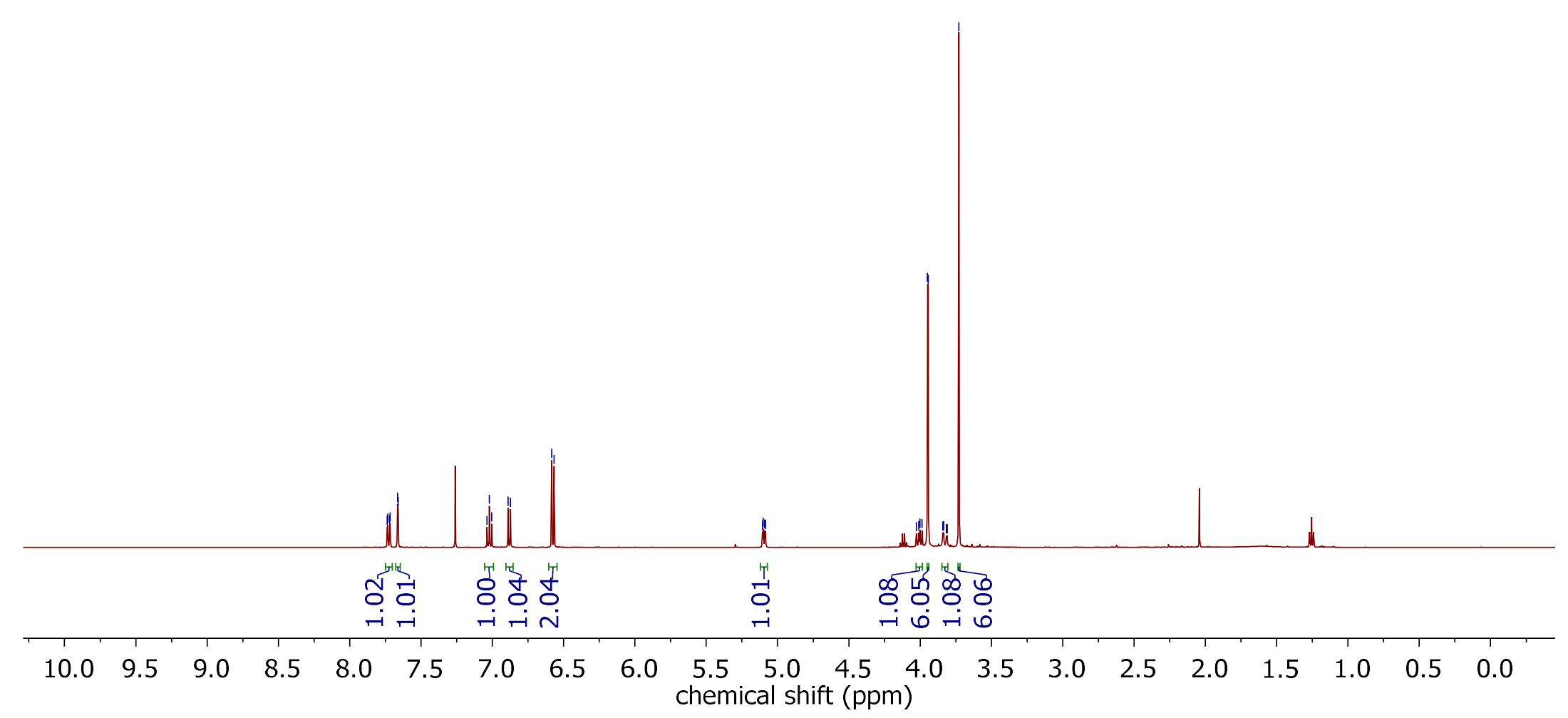




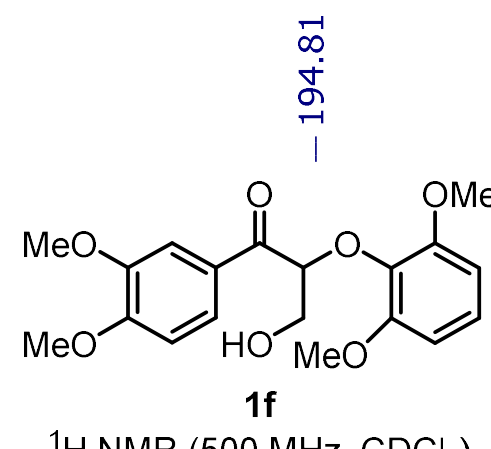

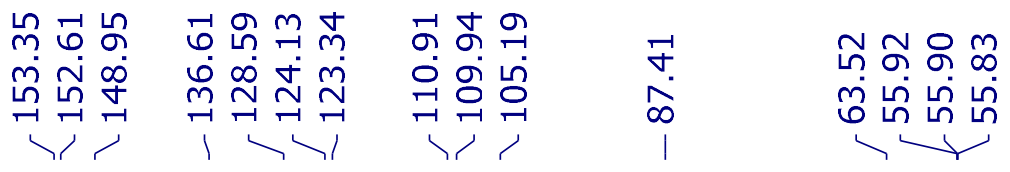

${ }^{1} \mathrm{H}$ NMR $\left(500 \mathrm{MHz}, \mathrm{CDCl}_{3}\right)$

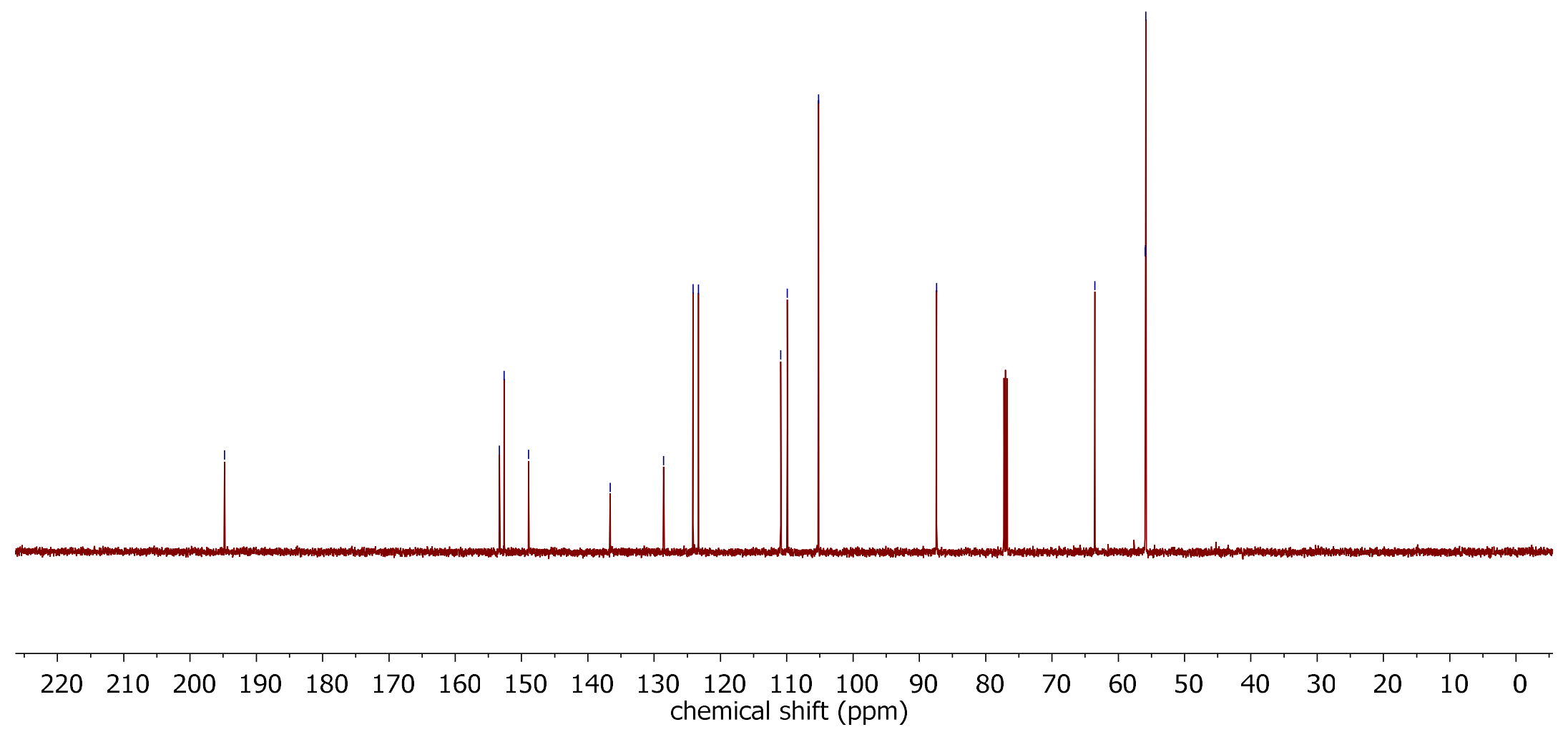




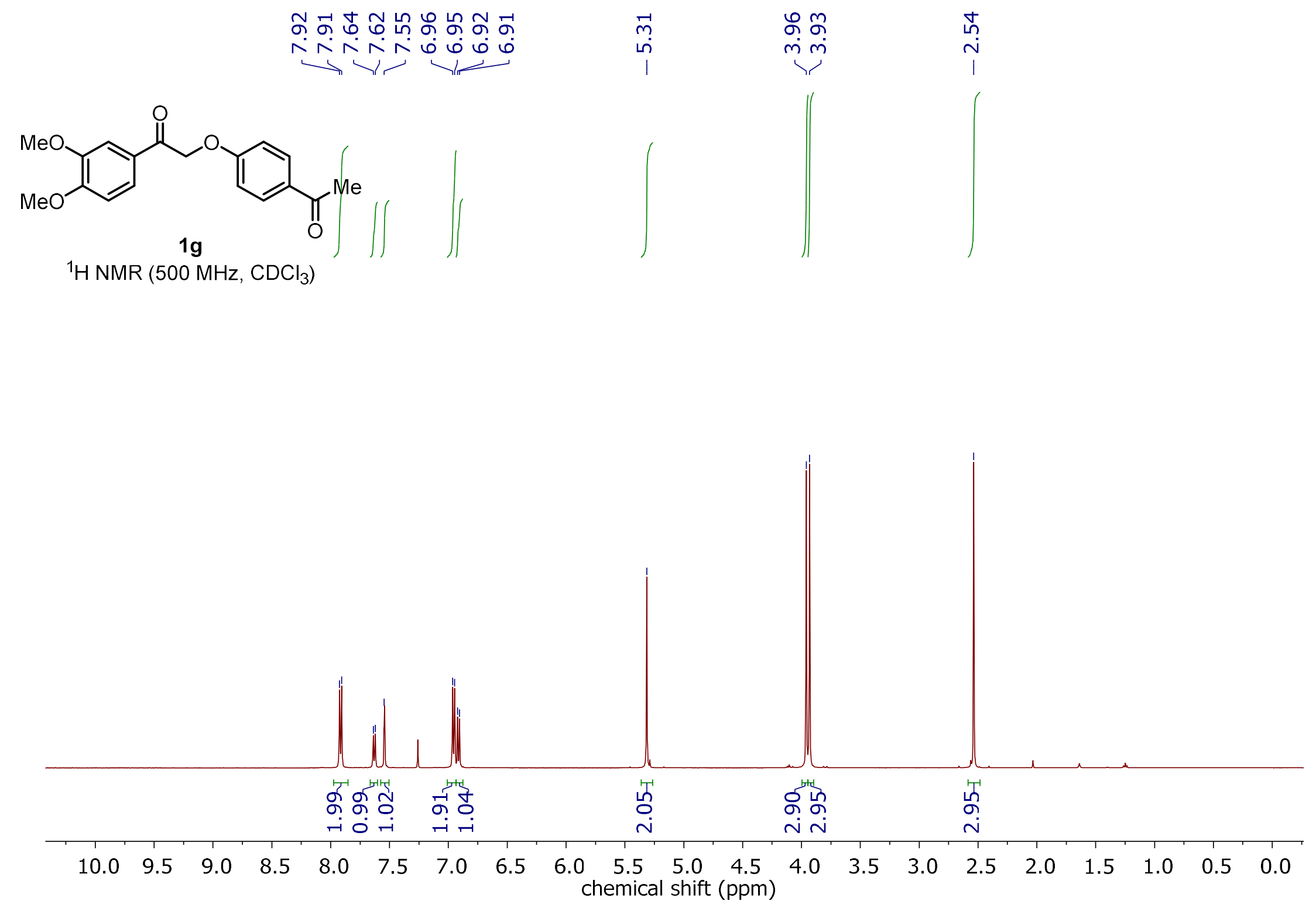



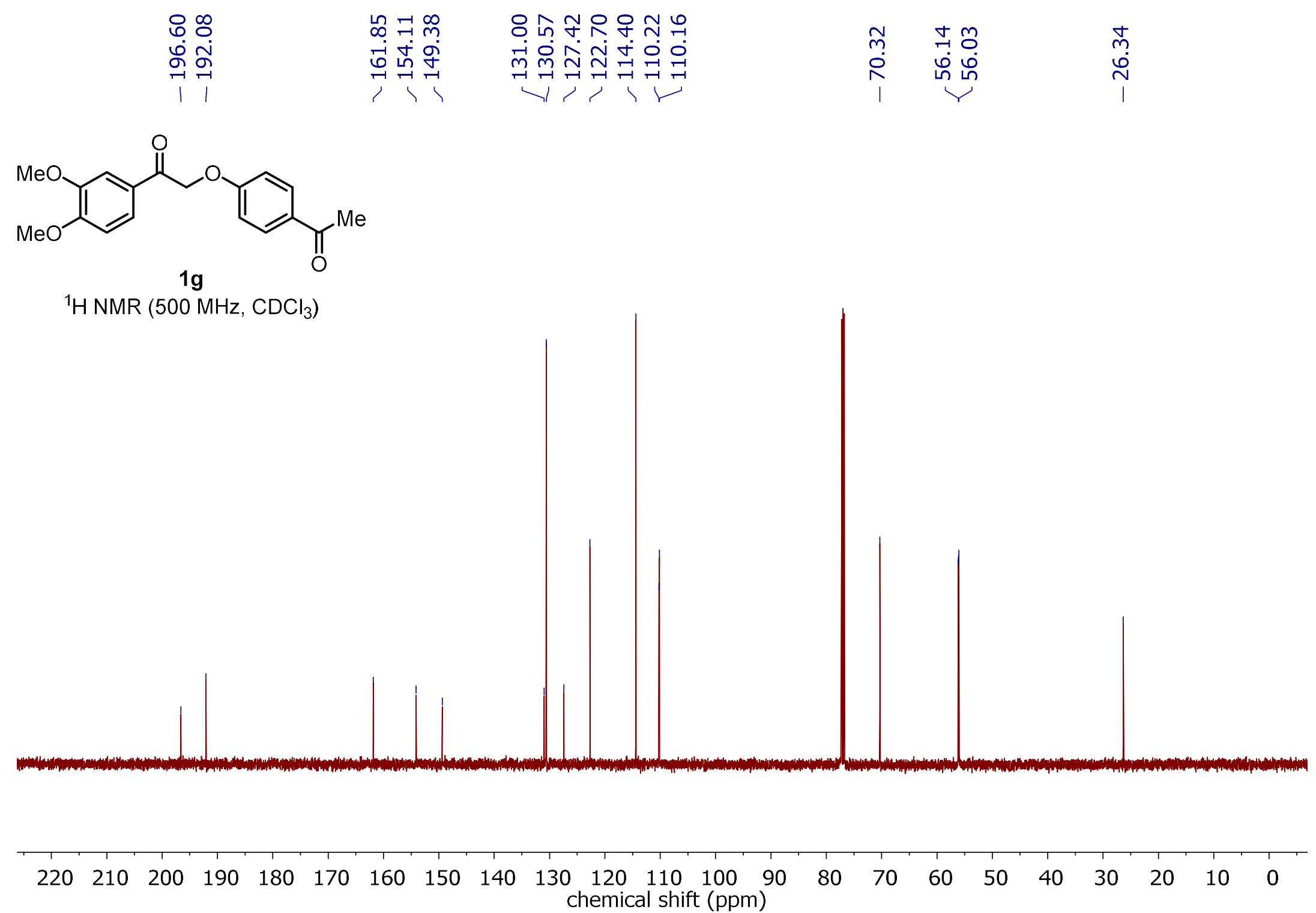
ळ

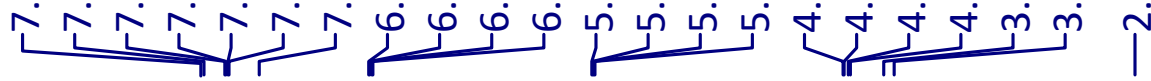

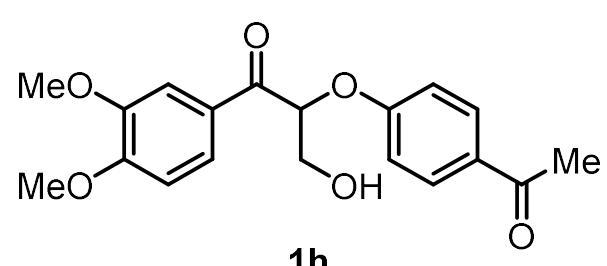

${ }^{1} \mathrm{H}$ NMR $\left(500 \mathrm{MHz}, \mathrm{CDCl}_{3}\right)$

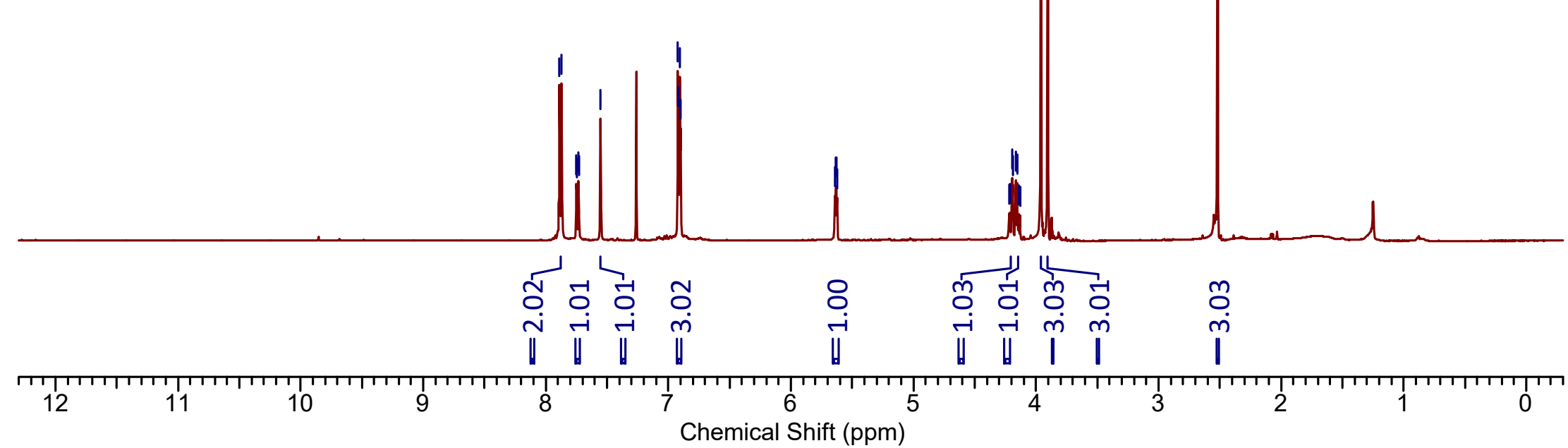




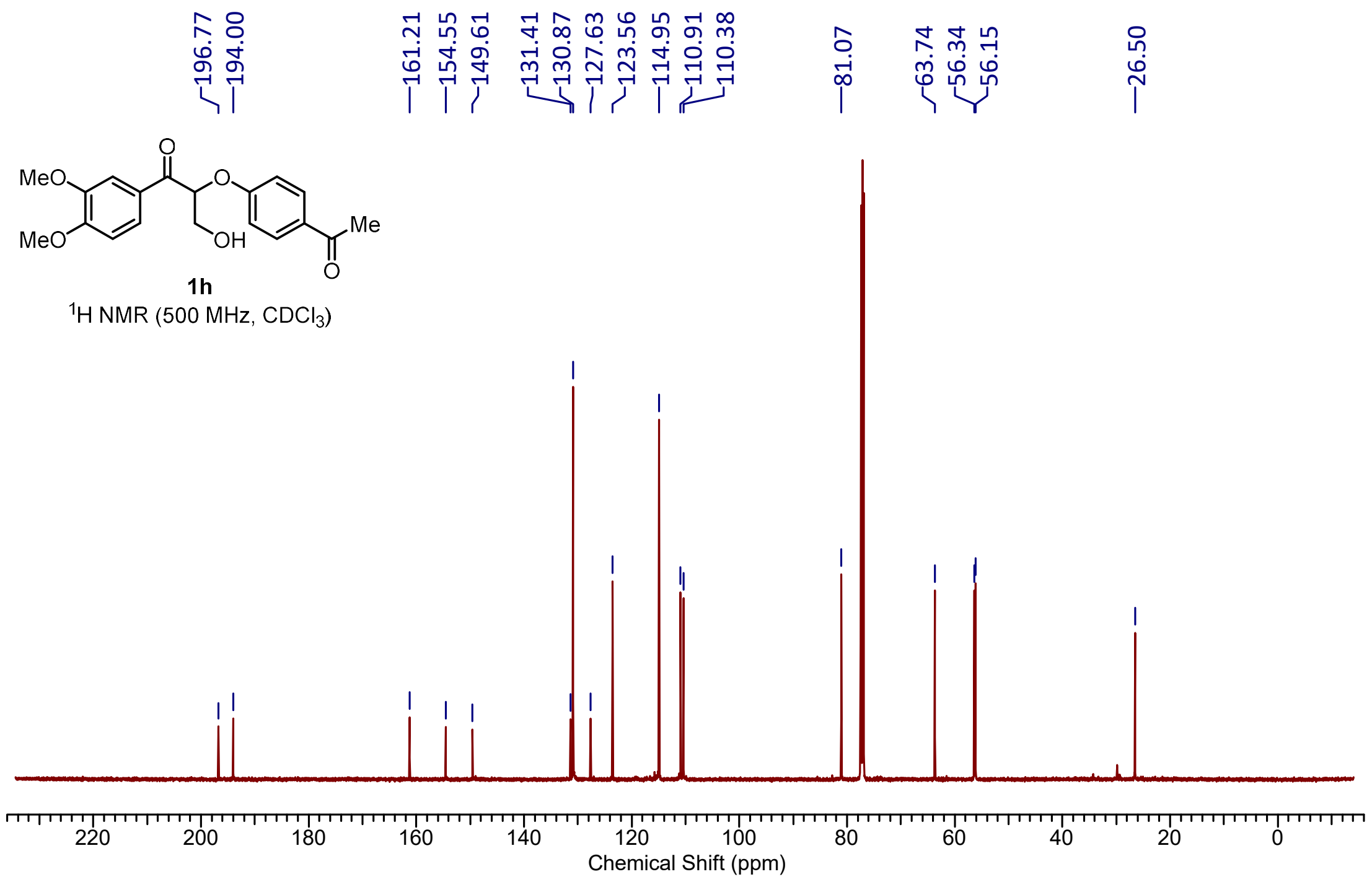




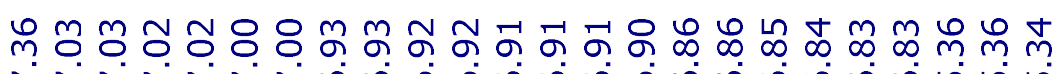
ヘ

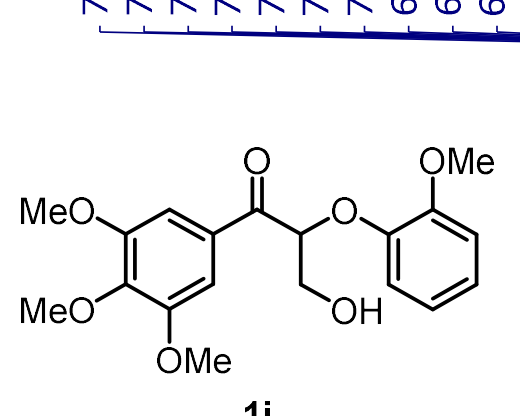

${ }^{1} \mathrm{H}$ NMR $\left(500 \mathrm{MHz}, \mathrm{CDCl}_{3}\right)$

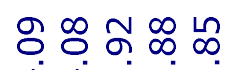

+ं

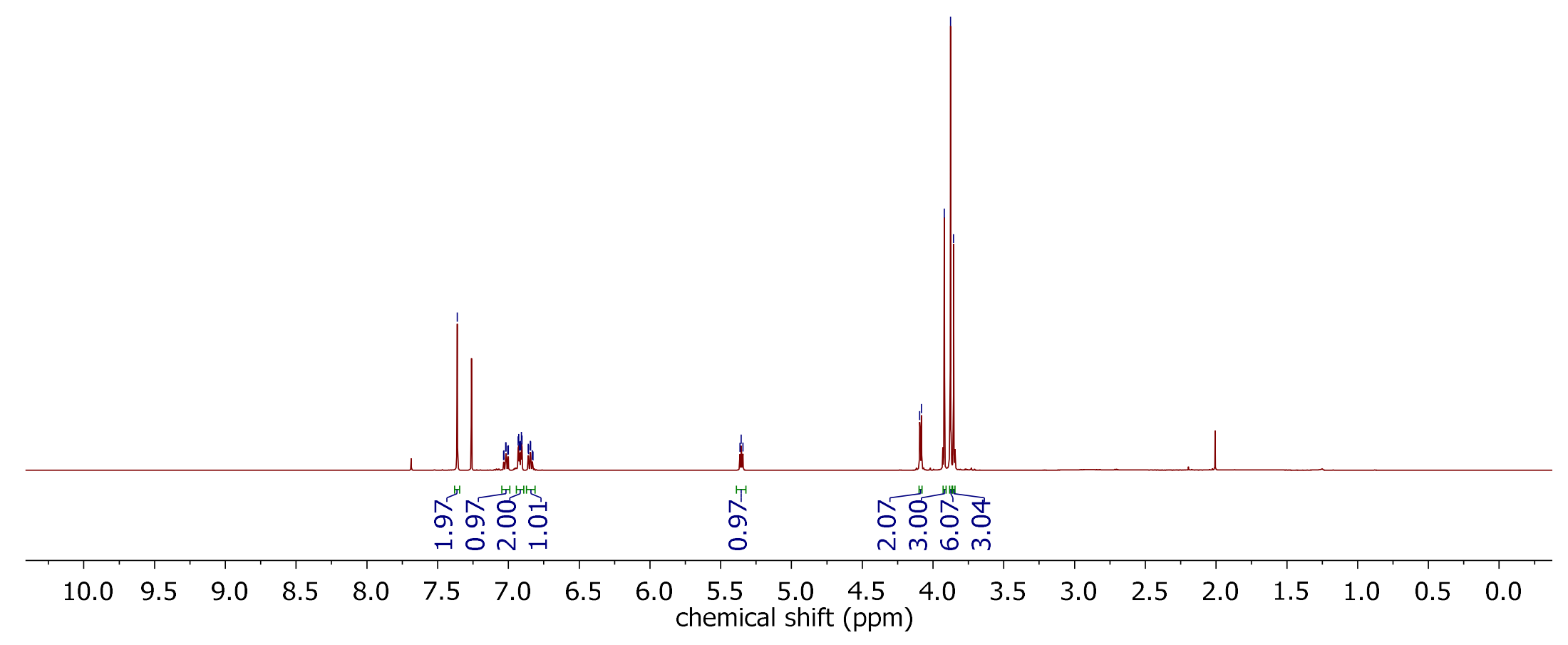




\begin{tabular}{|c|c|c|c|c|}
\hline $\begin{array}{l}\hat{n} \\
\text { nूं }\end{array}$ & 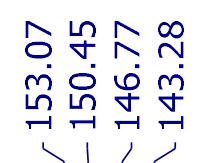 & 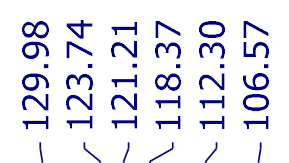 & $\begin{array}{l}8 \\
\dot{0} \\
\dot{\infty} \\
1\end{array}$ & 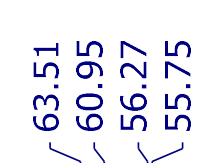 \\
\hline
\end{tabular}

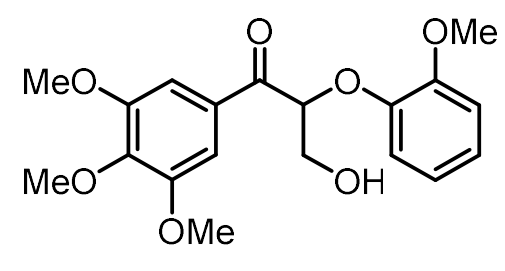

$1 \mathrm{i}$

${ }^{1} \mathrm{H}$ NMR $\left(500 \mathrm{MHz}, \mathrm{CDCl}_{3}\right)$

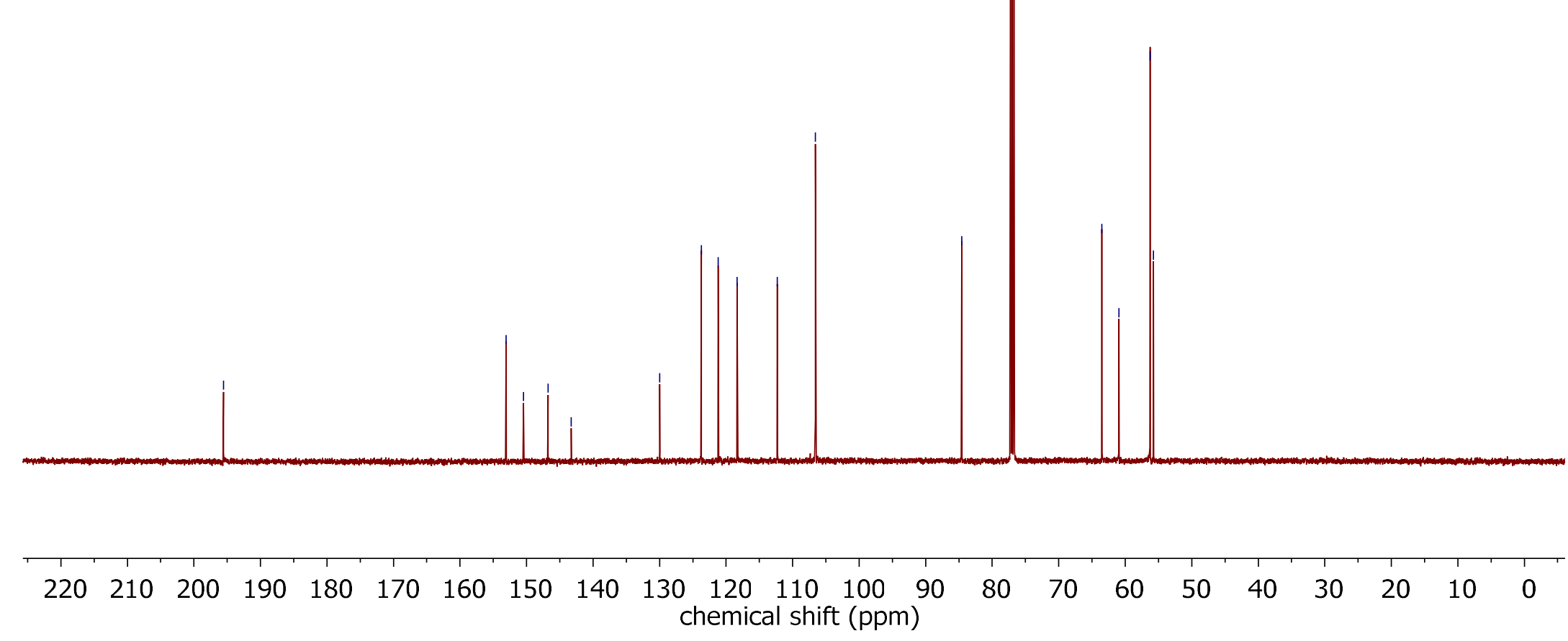


m

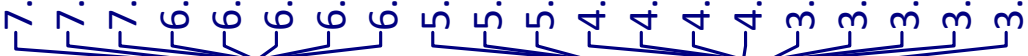
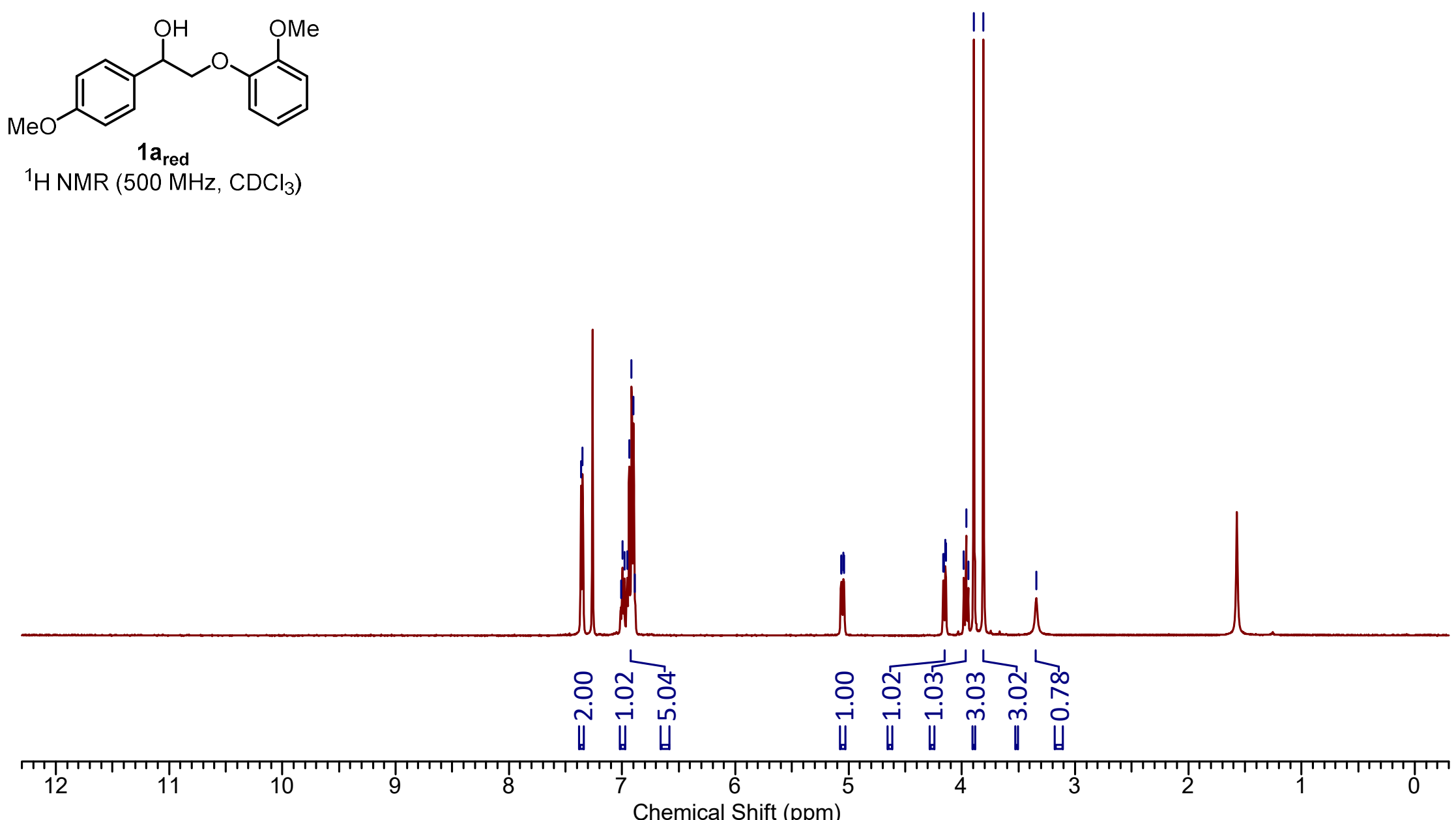

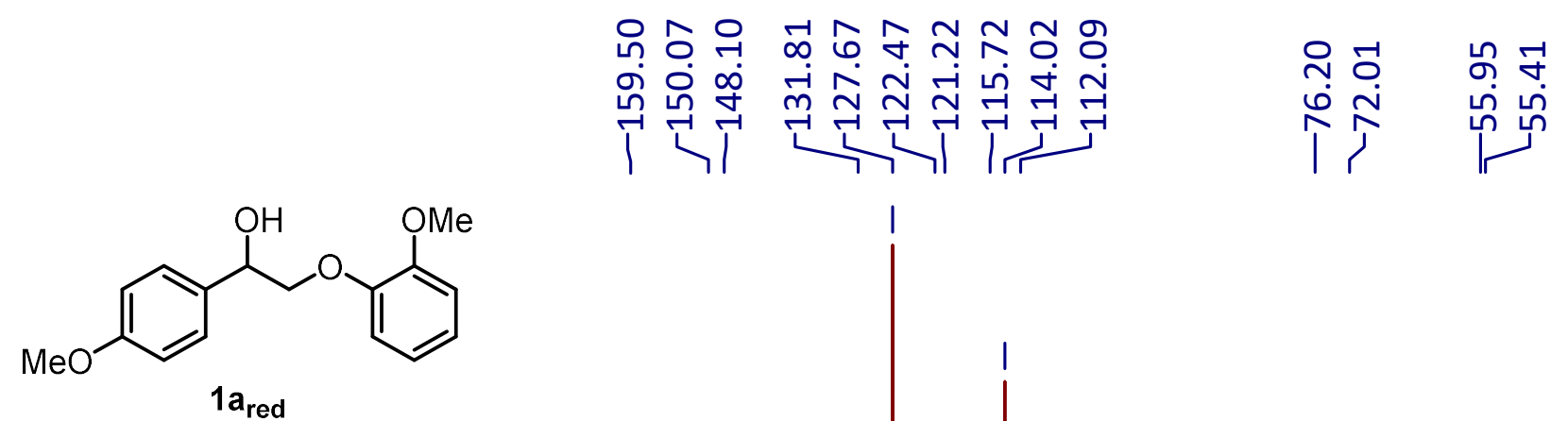

${ }^{1} \mathrm{H}$ NMR $\left(500 \mathrm{MHz}, \mathrm{CDCl}_{3}\right)$

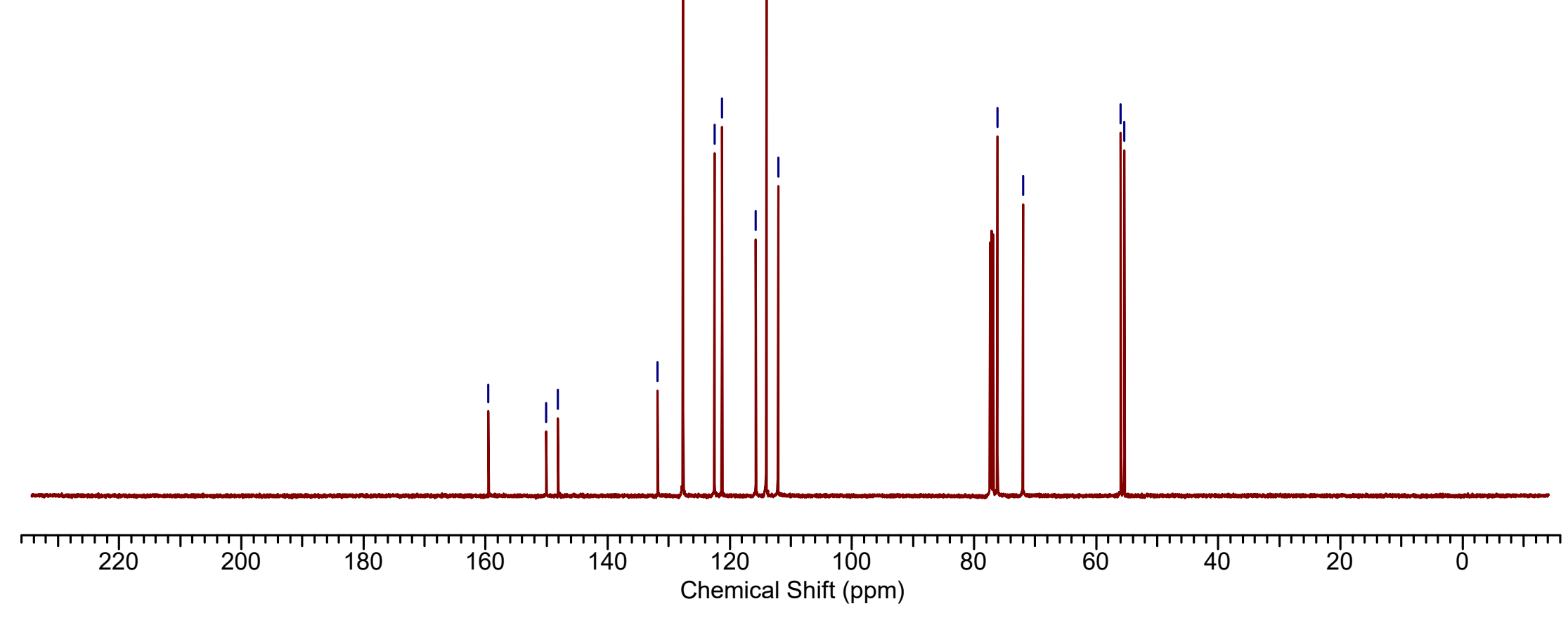




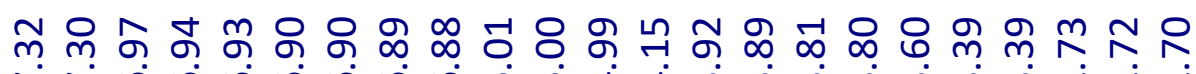

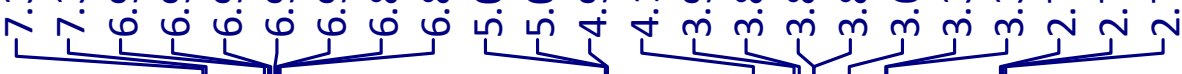
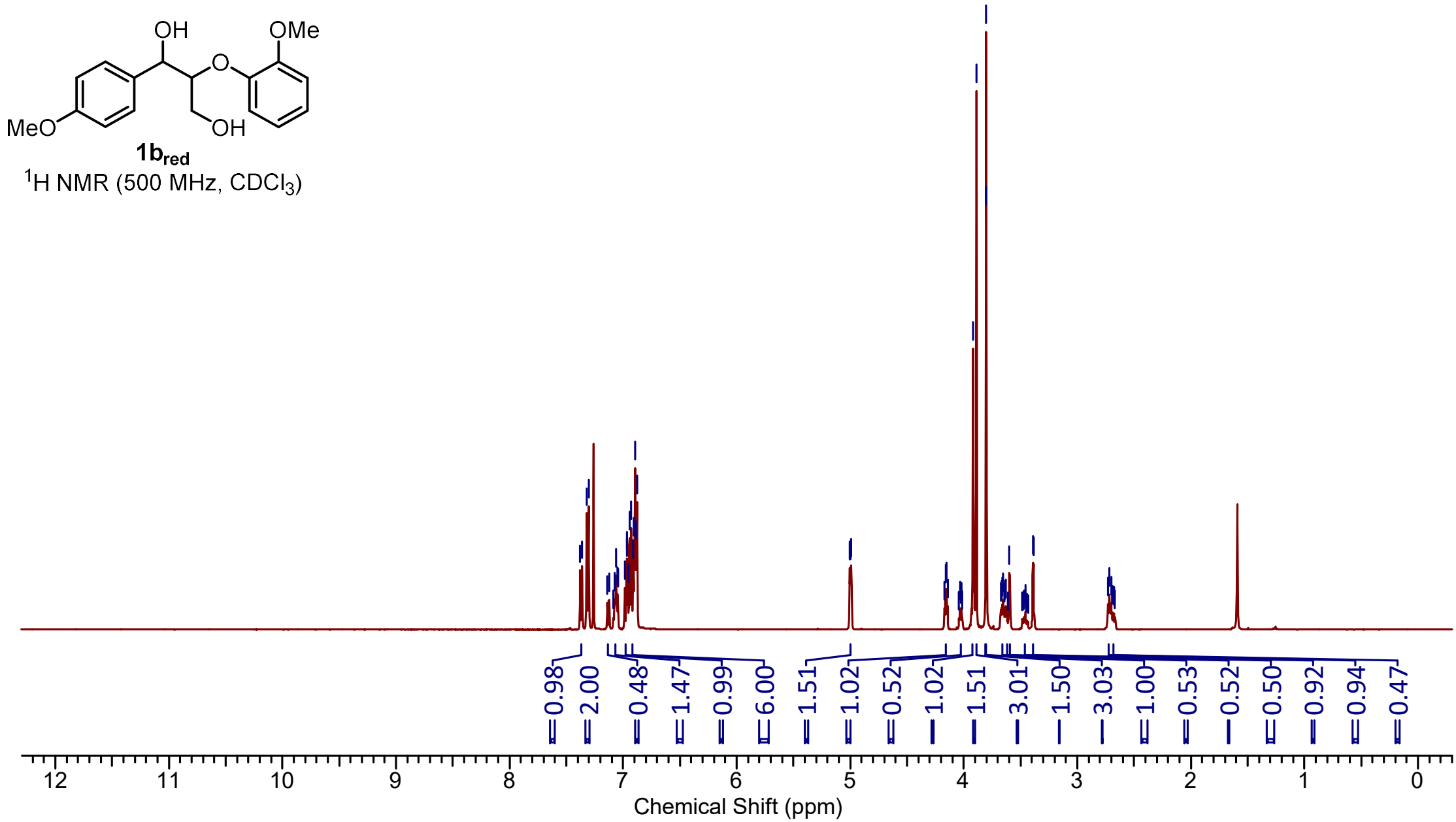


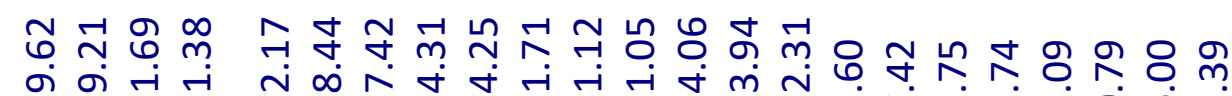

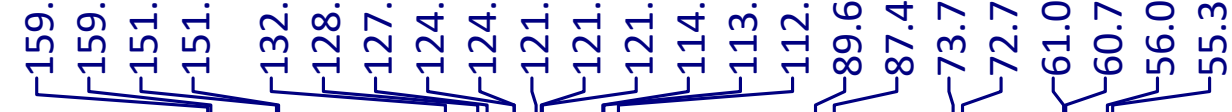

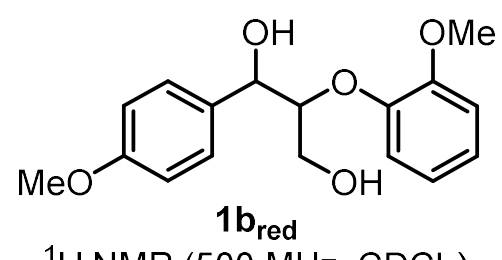

${ }^{1} \mathrm{H}$ NMR $\left(500 \mathrm{MHz}, \mathrm{CDCl}_{3}\right)$

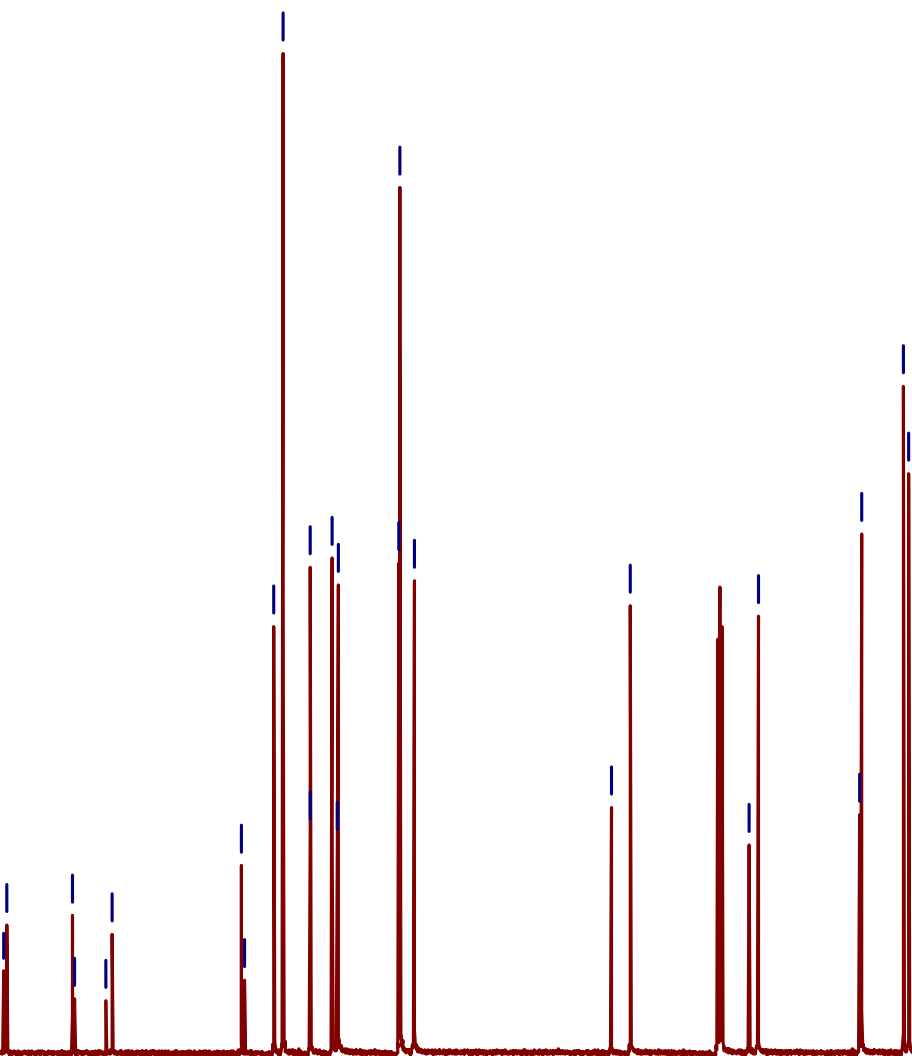

220

220

200

180

160

140

120

$100 \quad 80$

80

60

40

20

0 


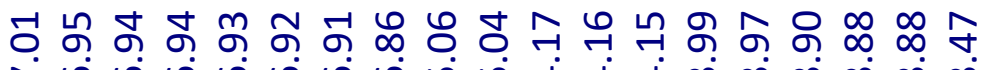

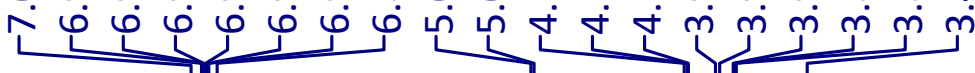
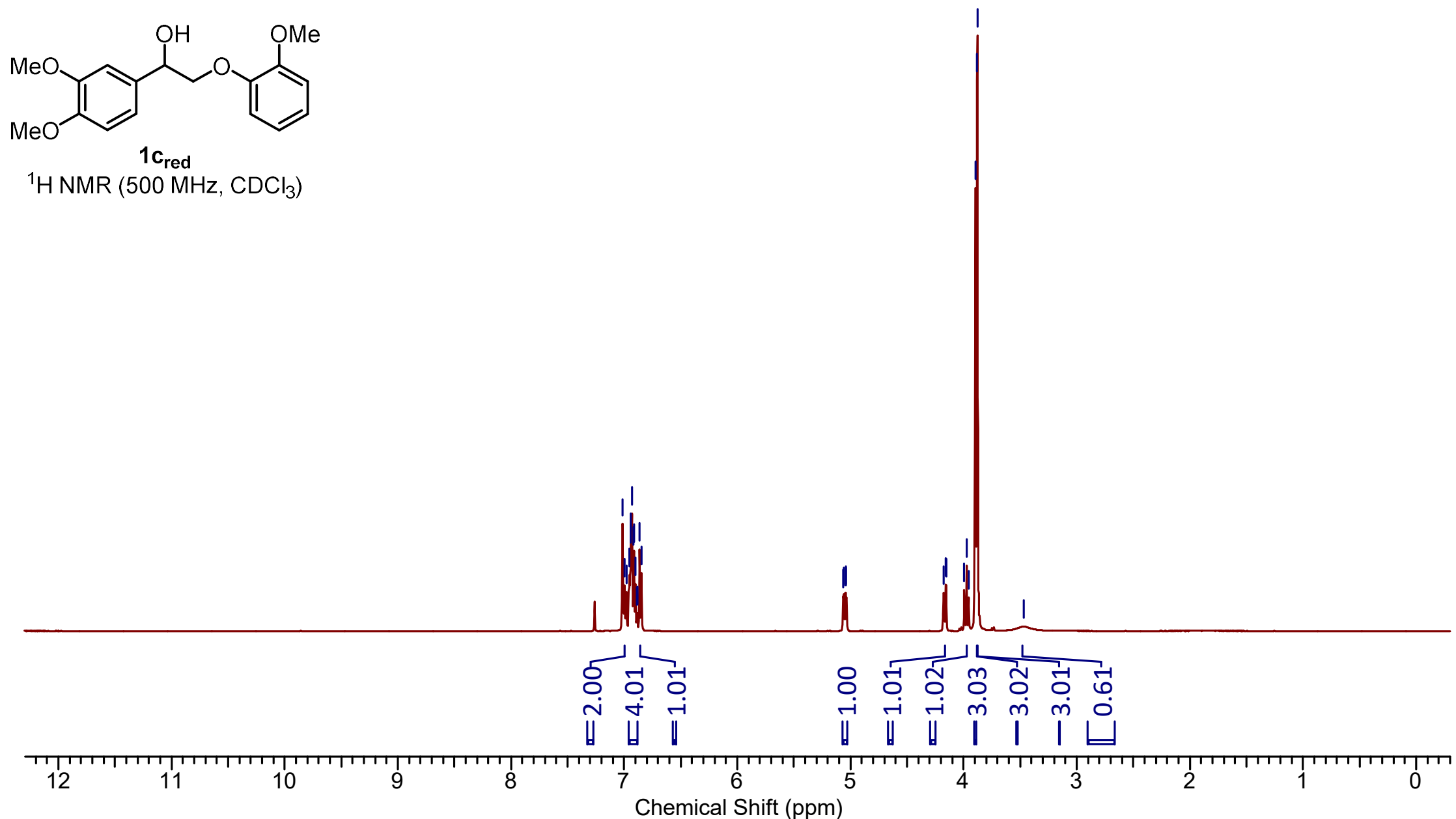


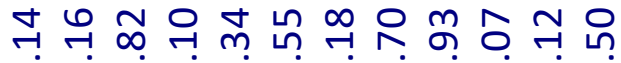

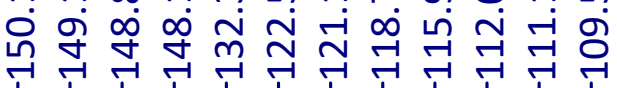

m 궁

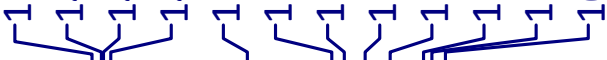

மำ

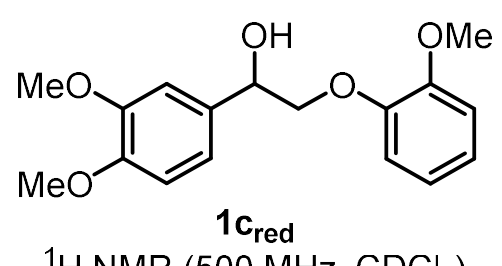

${ }^{1} \mathrm{H}$ NMR $\left(500 \mathrm{MHz}, \mathrm{CDCl}_{3}\right)$

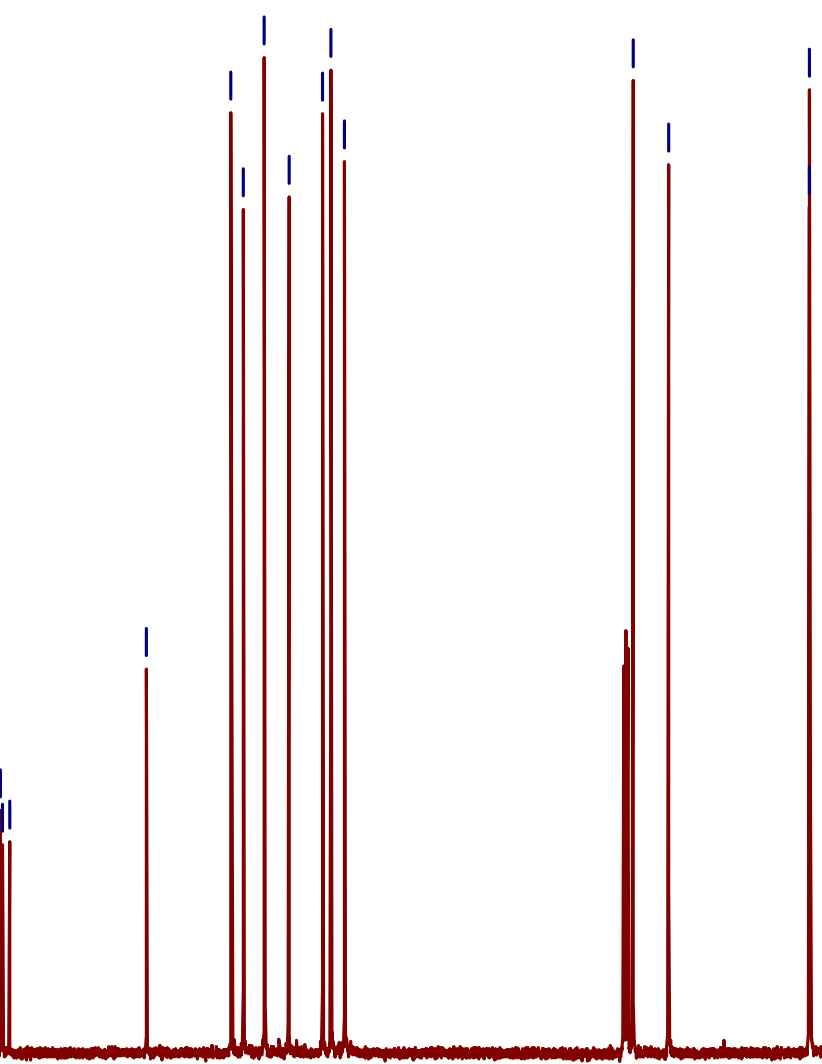

1)

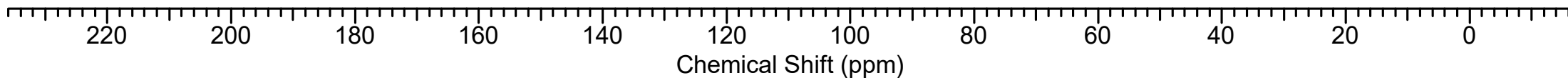




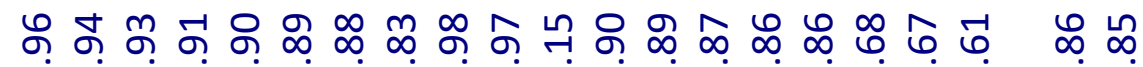

$\dot{\varphi} \dot{\varphi} \dot{\varphi} \dot{\varphi} \dot{\varphi} \dot{\varphi} \dot{0}$

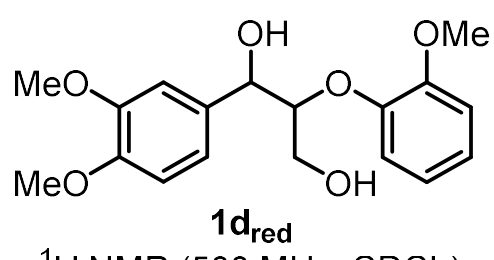

${ }^{1} \mathrm{H}$ NMR $\left(500 \mathrm{MHz}, \mathrm{CDCl}_{3}\right)$

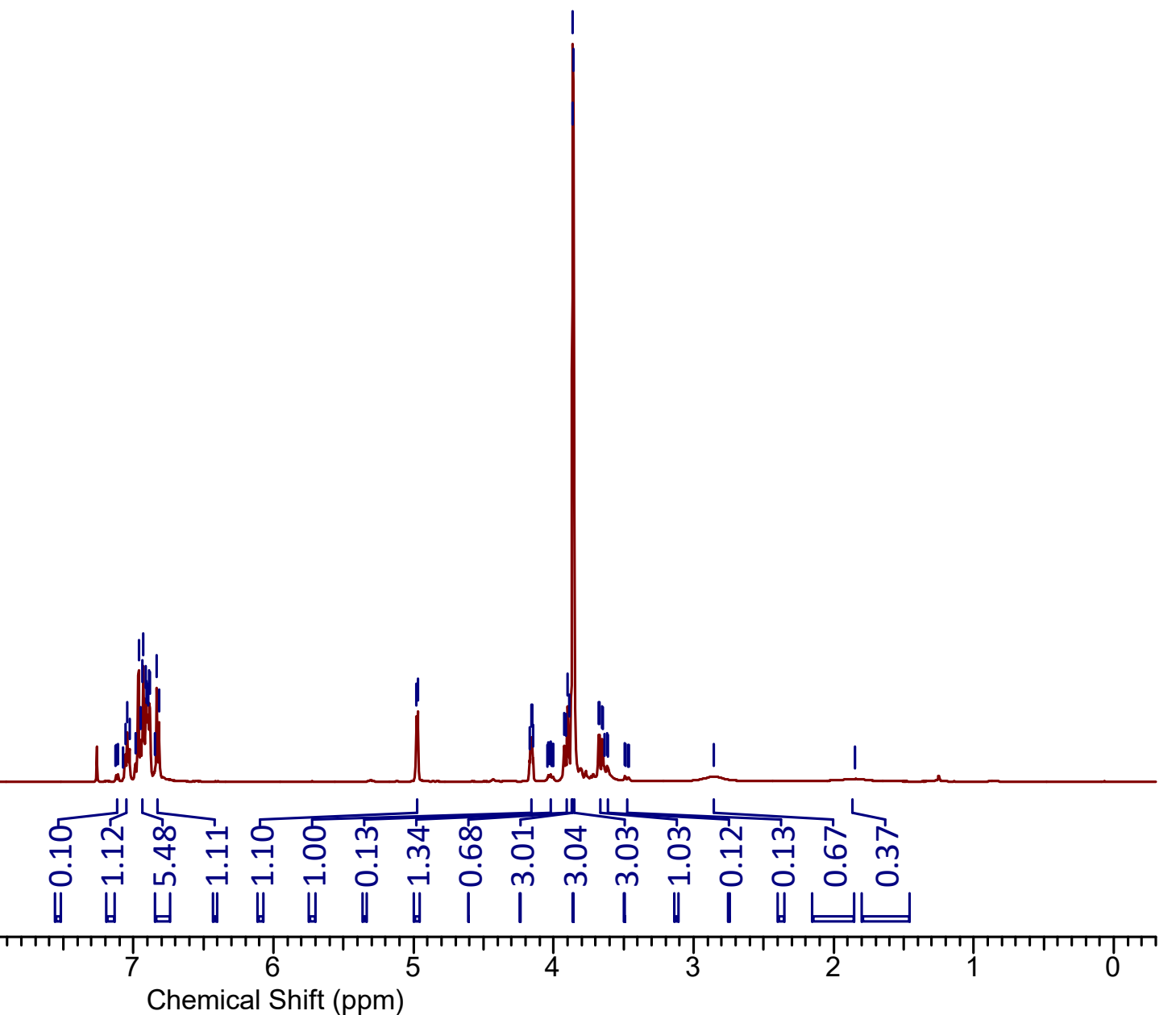


늑 군

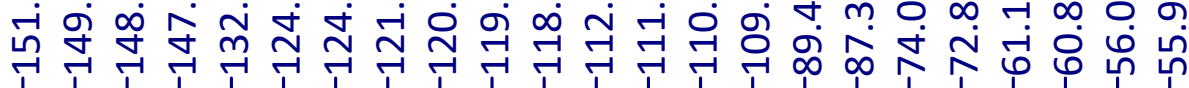

L

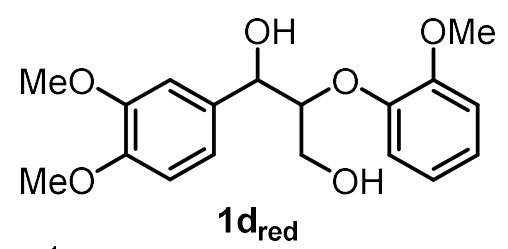

${ }^{1} \mathrm{H}$ NMR $\left(500 \mathrm{MHz}, \mathrm{CDCl}_{3}\right)$

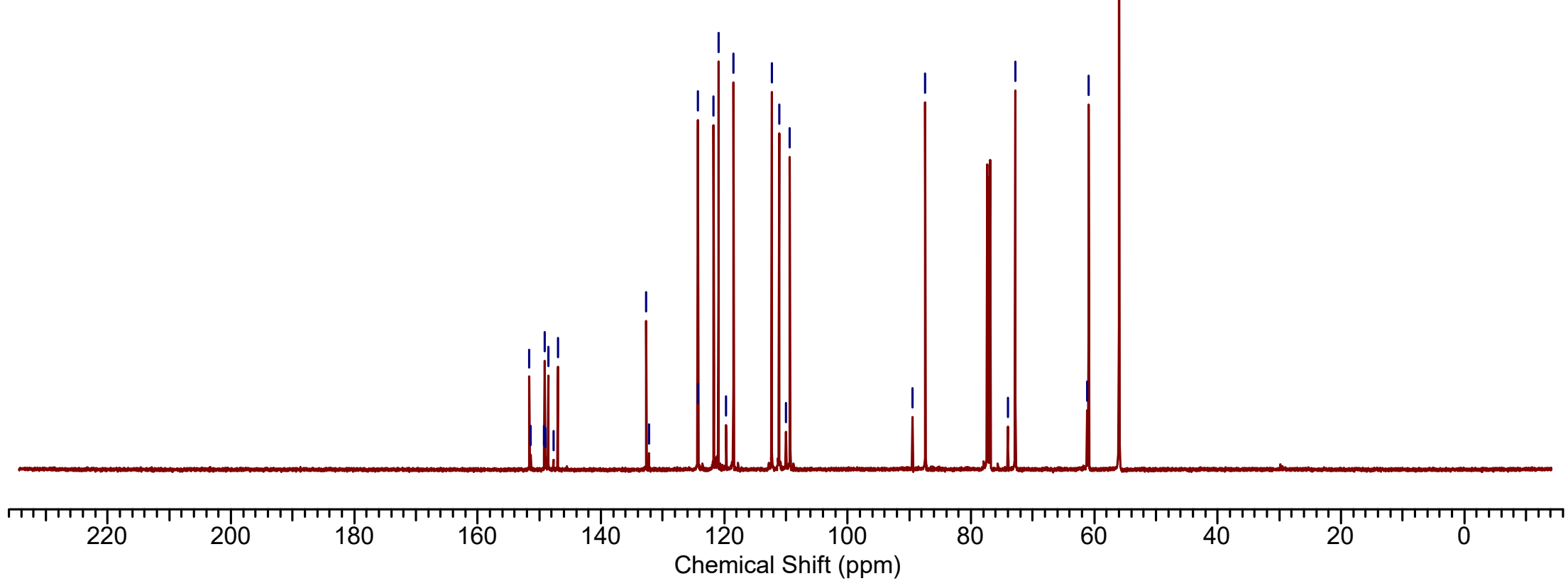




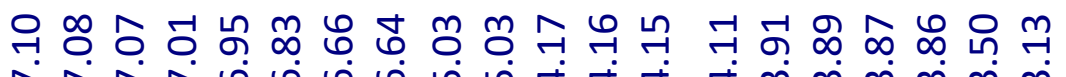

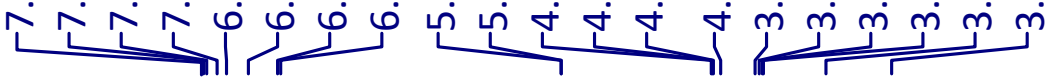
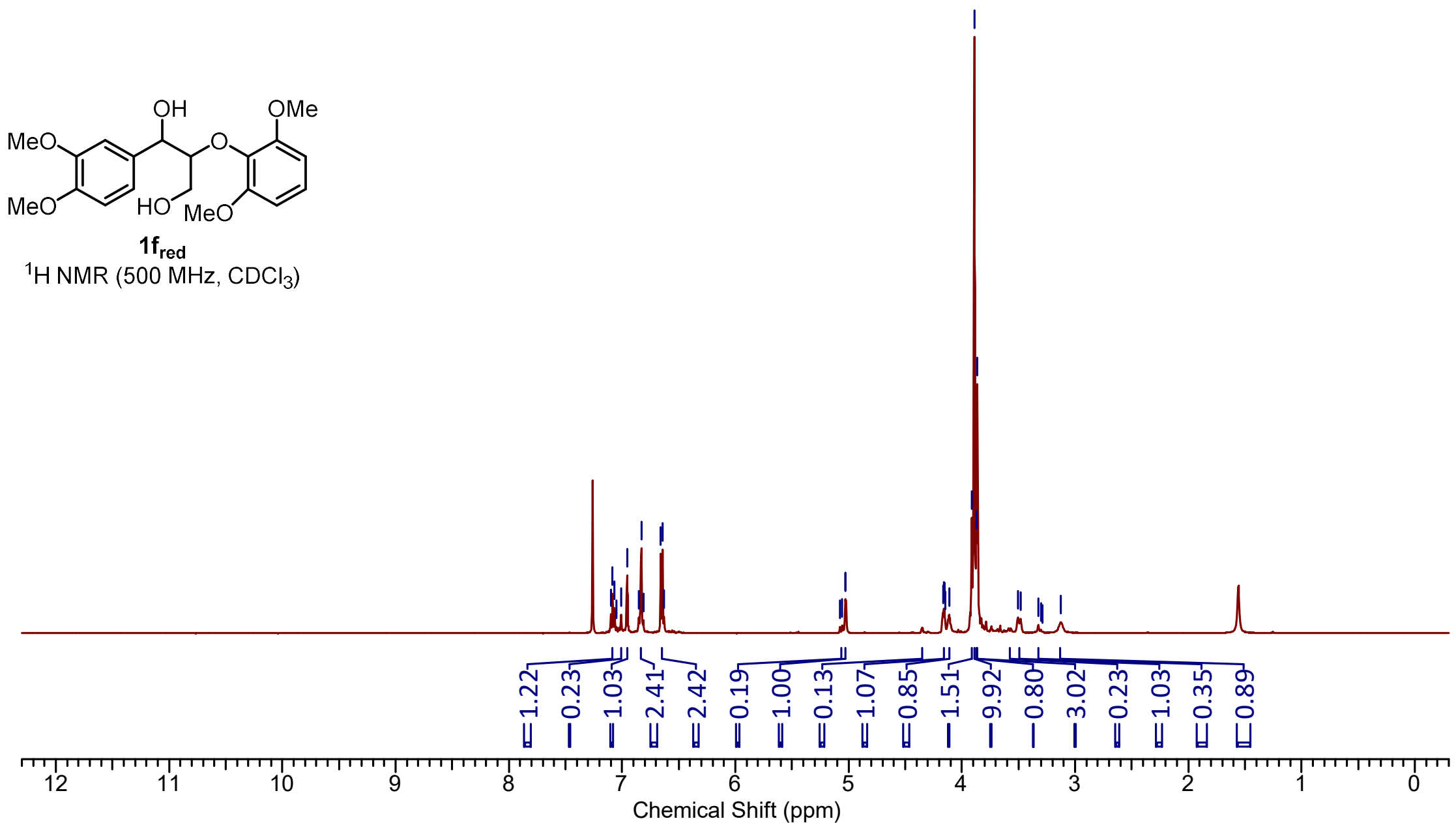


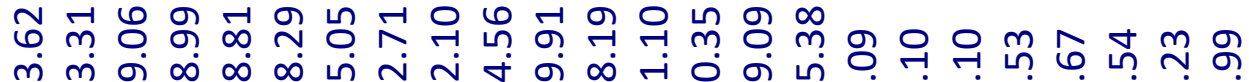

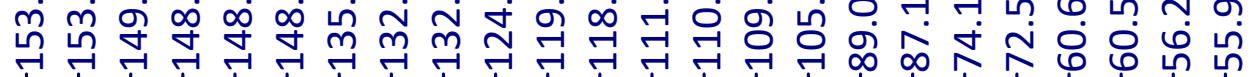
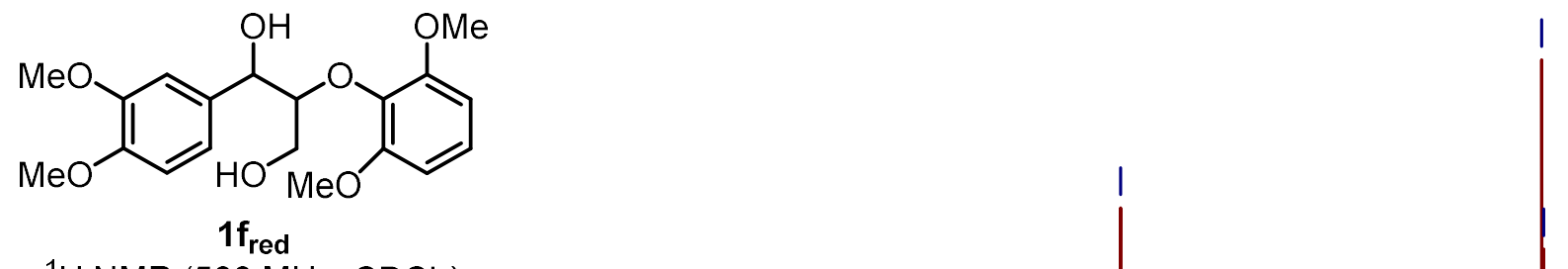

${ }^{1} \mathrm{H} \mathrm{NMR}\left(500 \mathrm{MHz}, \mathrm{CDCl}_{3}\right)$

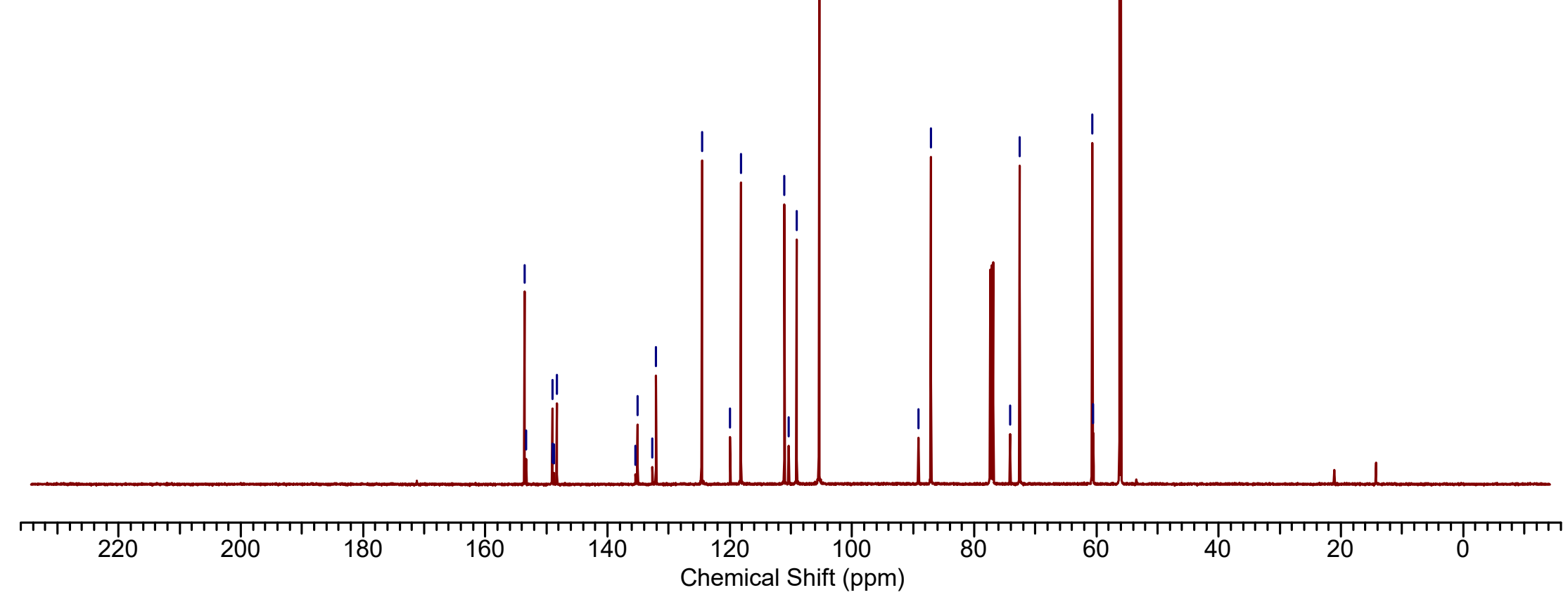




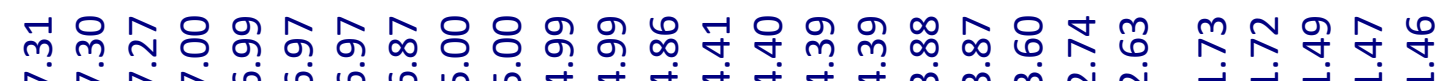

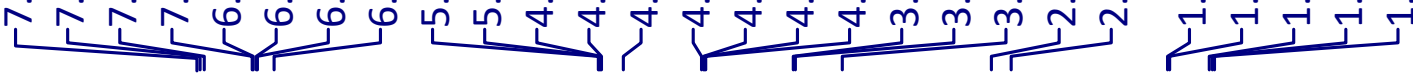

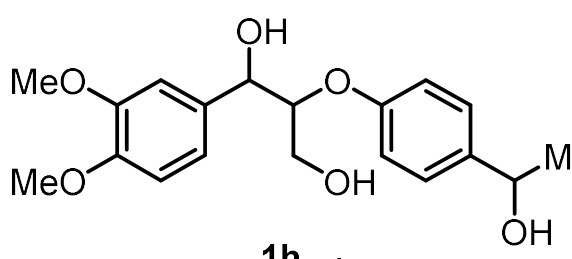

$1 \mathrm{~h}_{\text {red }}$

${ }^{1} \mathrm{H} \mathrm{NMR}\left(500 \mathrm{MHz}, \mathrm{CDCl}_{3}\right)$

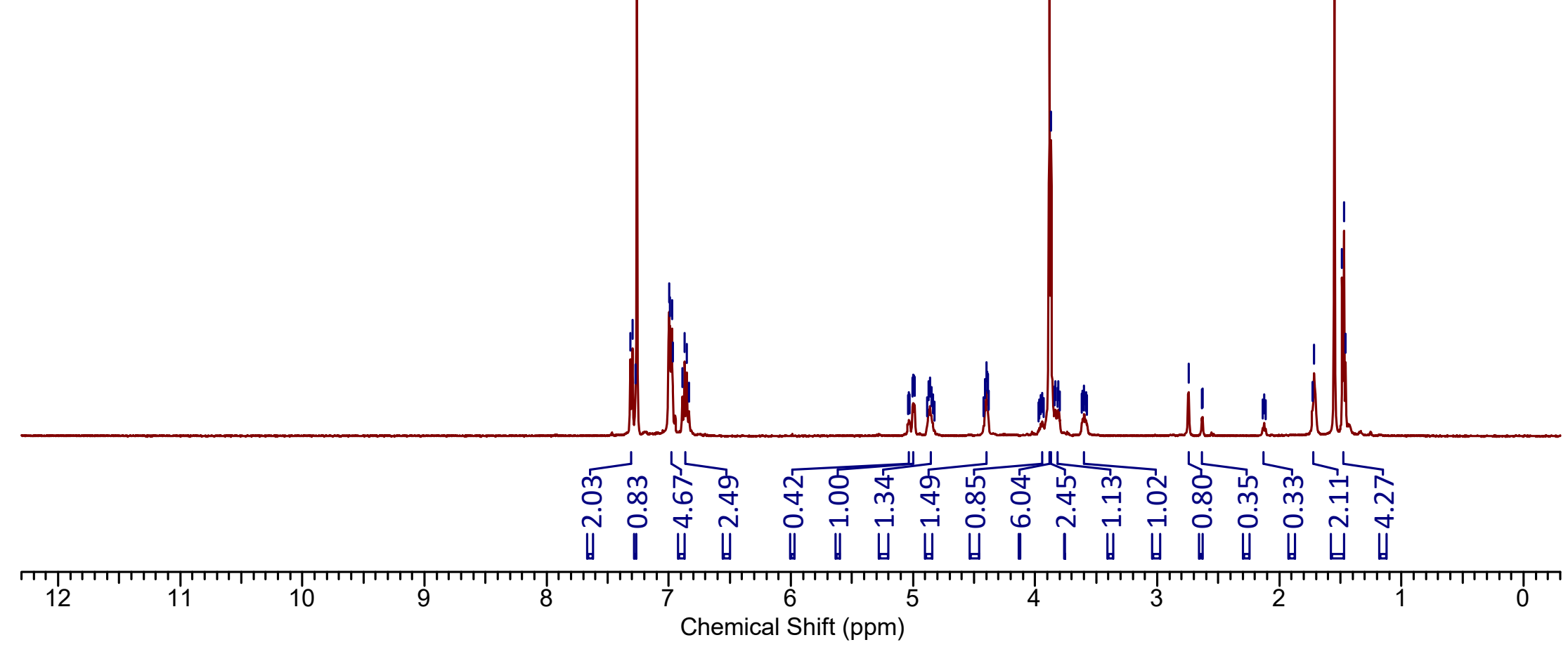




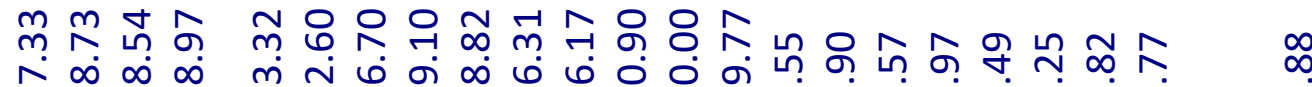

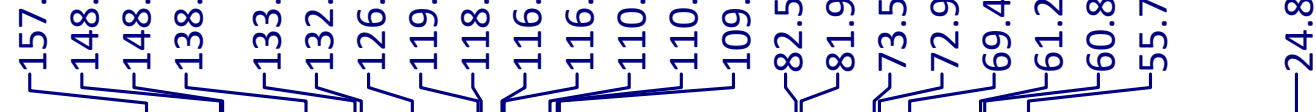

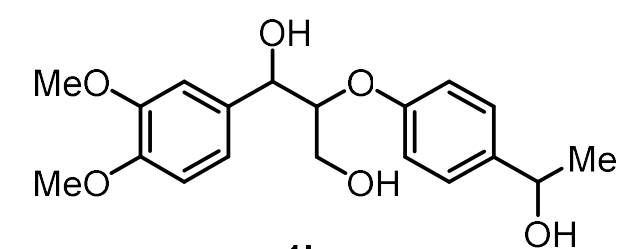

$1 \mathrm{~h}_{\text {red }}$

${ }^{1} \mathrm{H}$ NMR $\left(500 \mathrm{MHz}, \mathrm{CDCl}_{3}\right)$

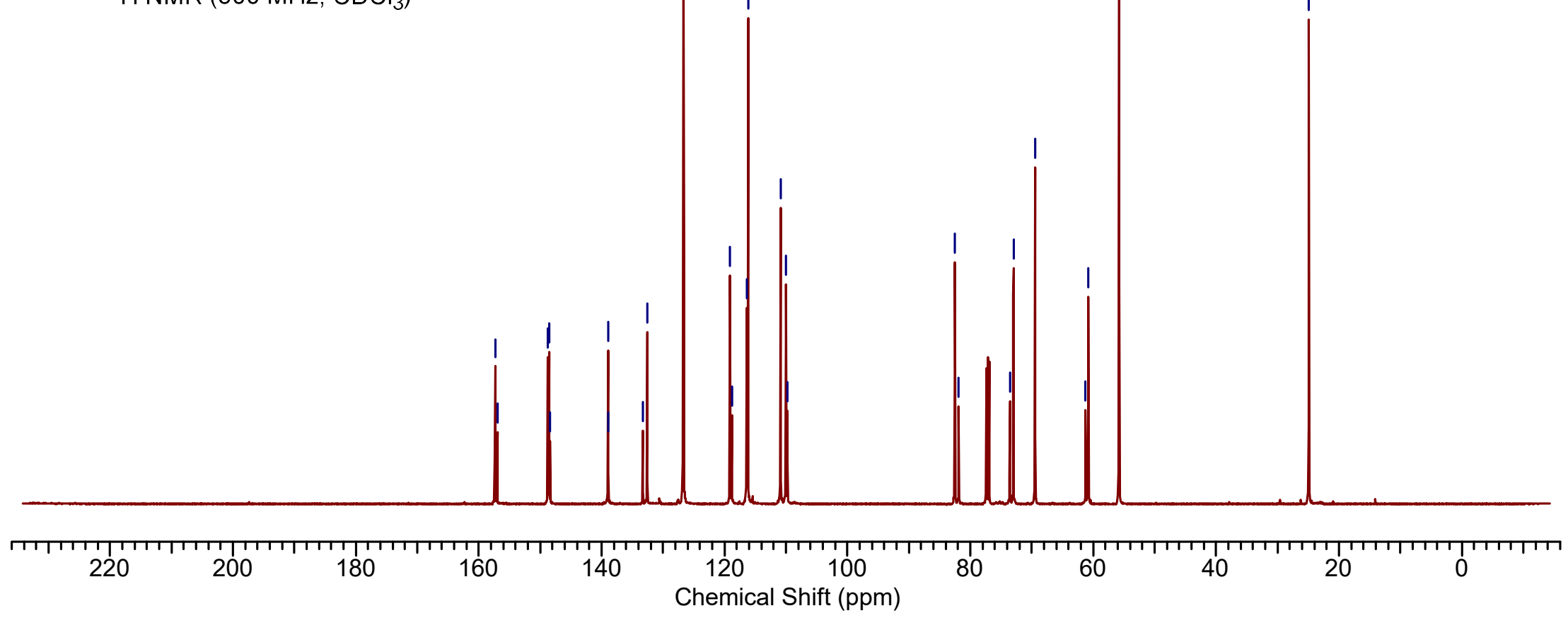




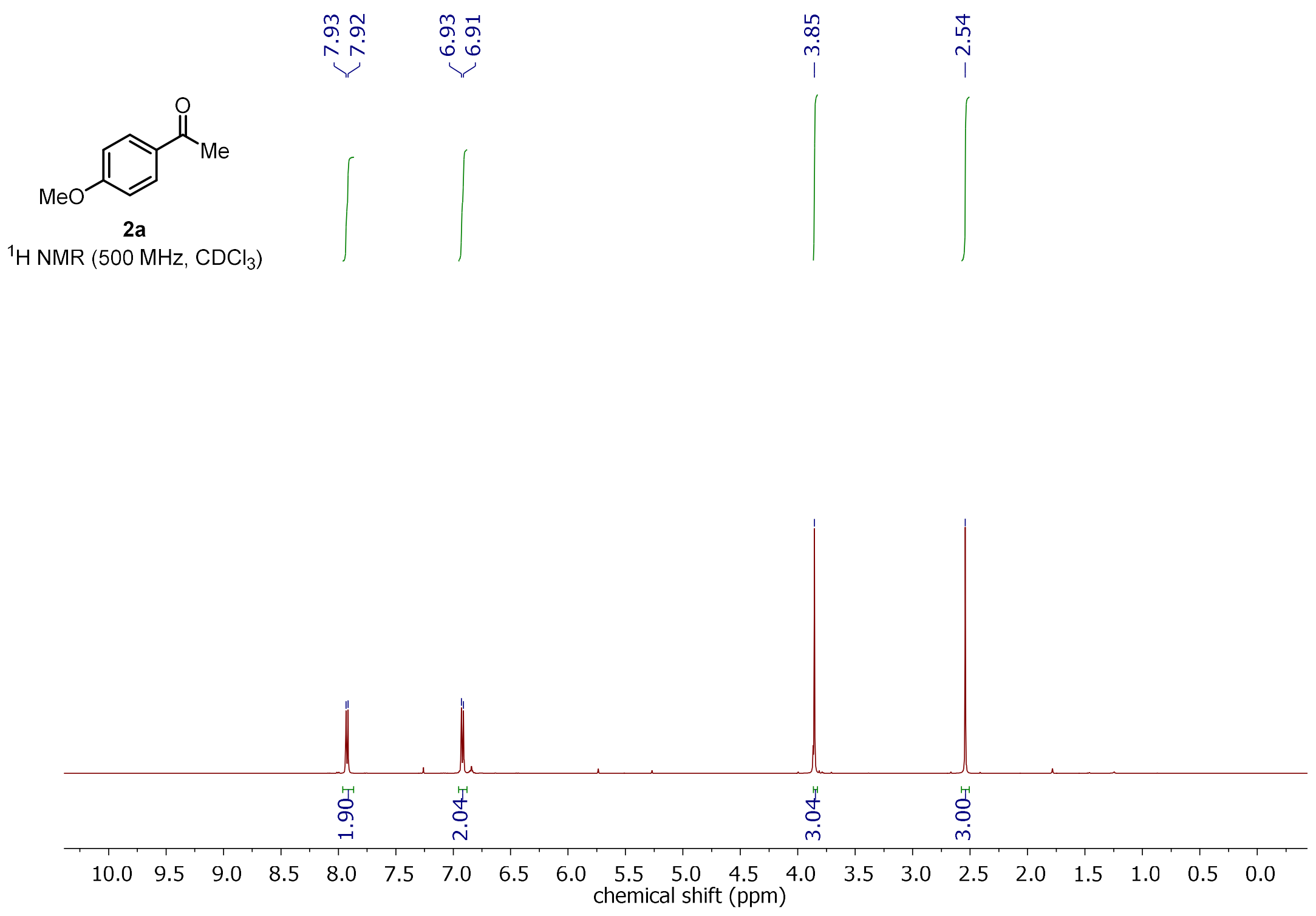




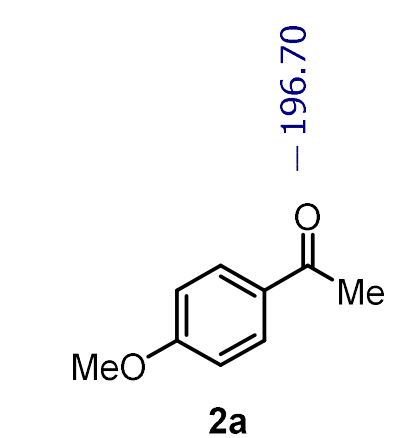

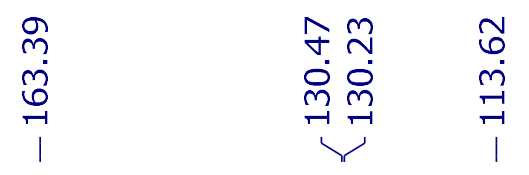

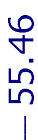

$\stackrel{\stackrel{n}{m}}{\stackrel{\leftrightarrow}{\sim}}$

${ }^{1} \mathrm{H}$ NMR $\left(500 \mathrm{MHz}, \mathrm{CDCl}_{3}\right)$

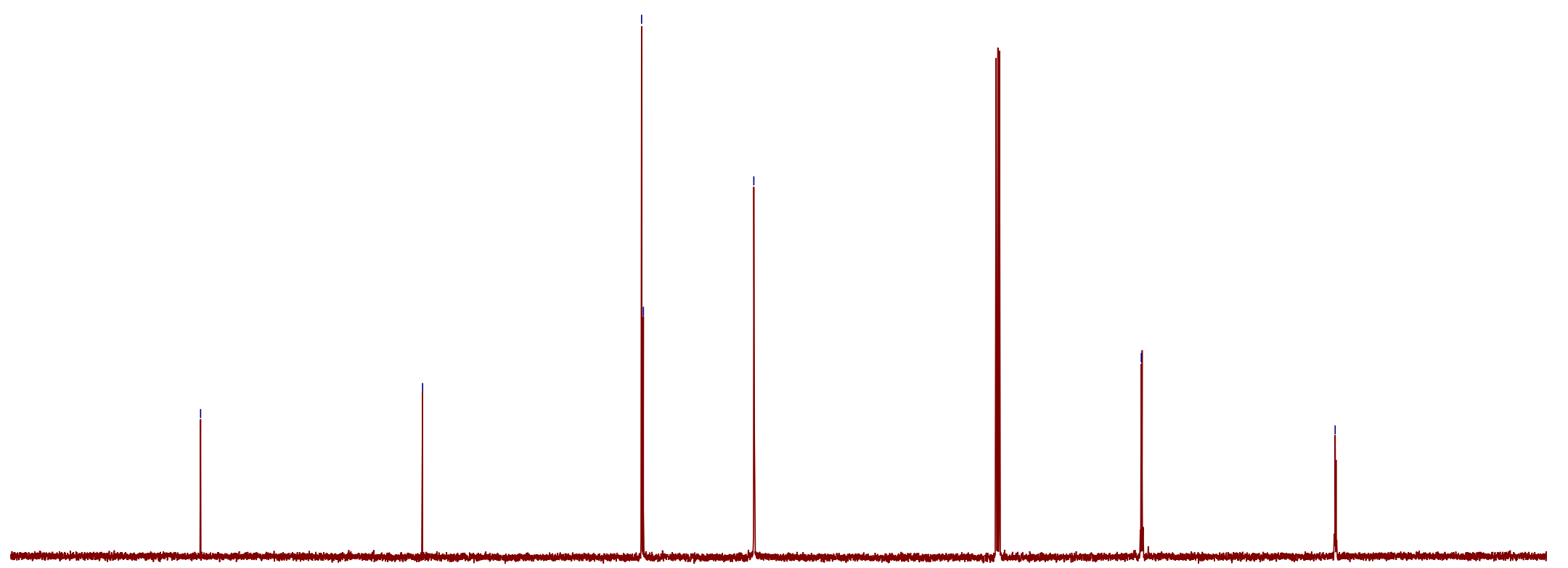

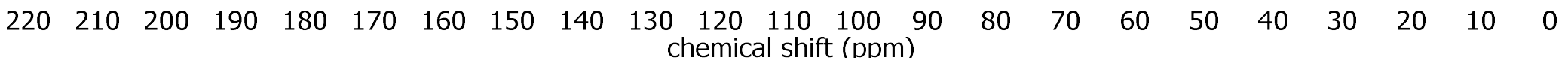



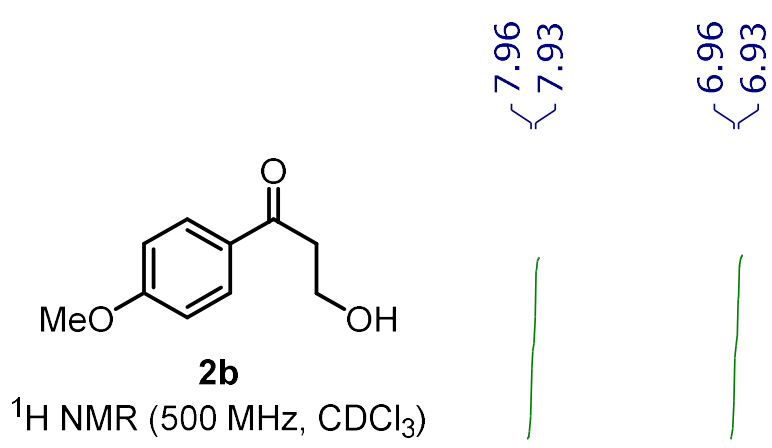

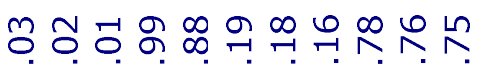

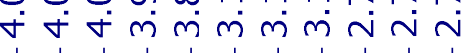

,
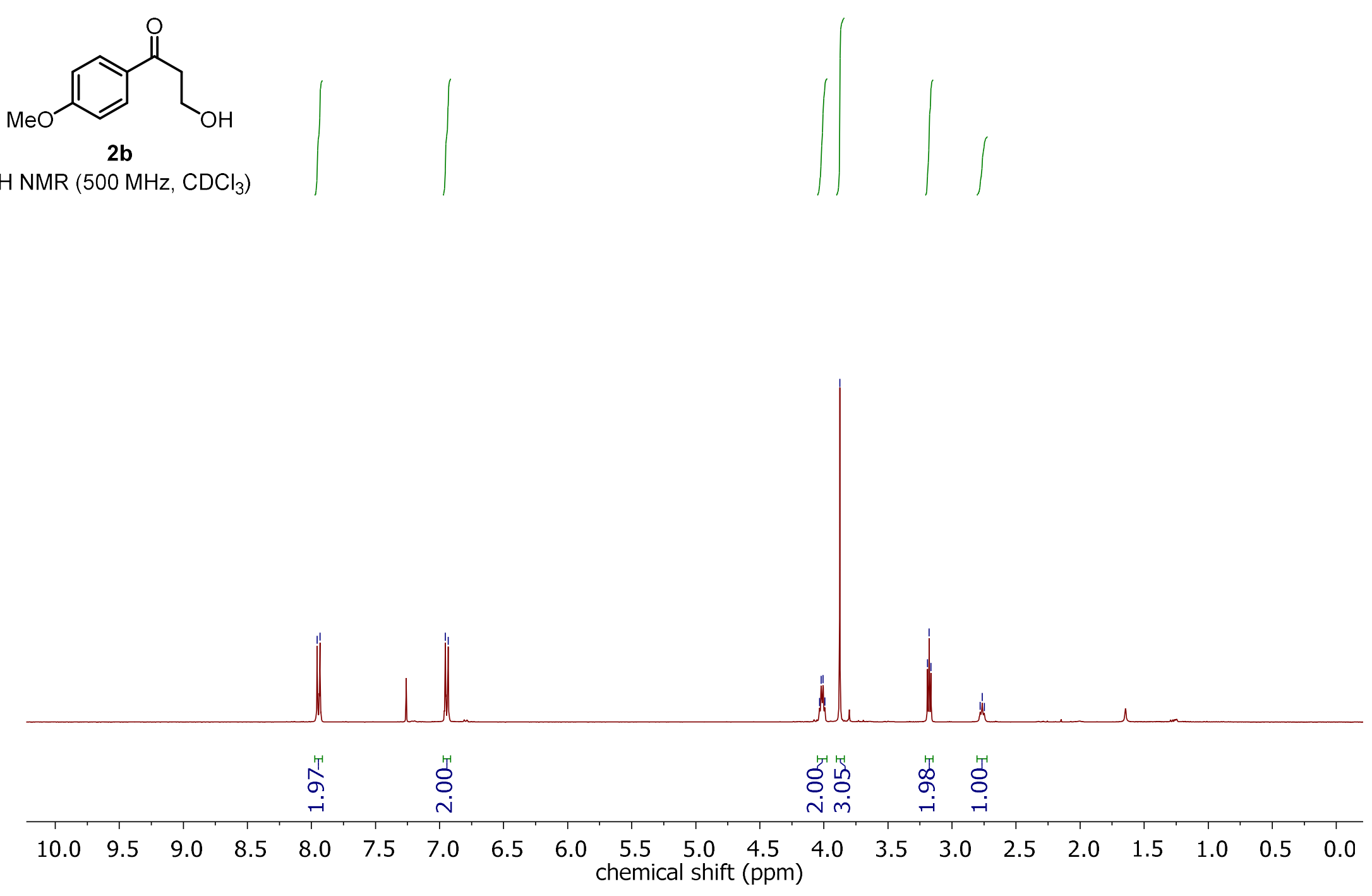

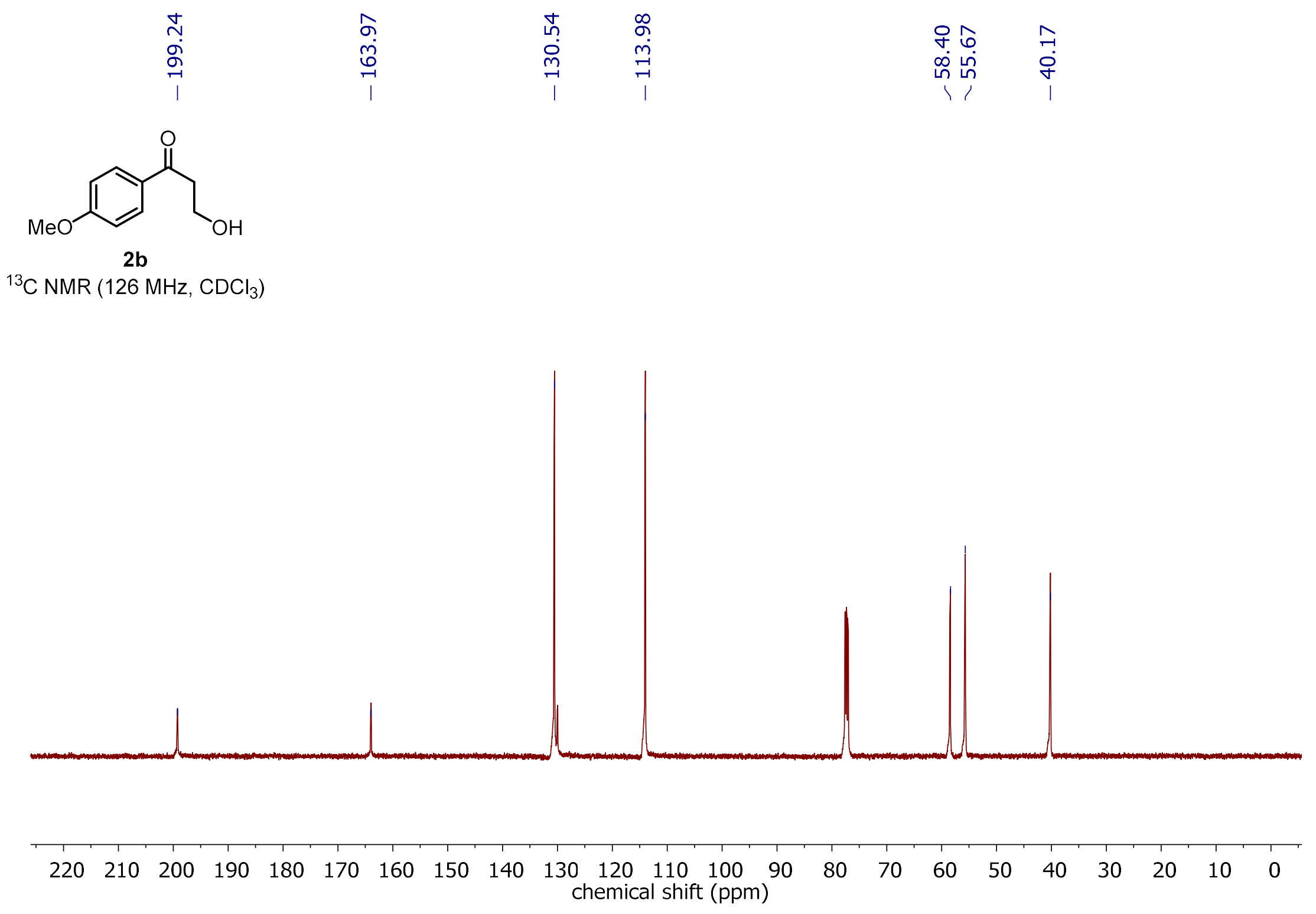
กิ่

NNNNNN

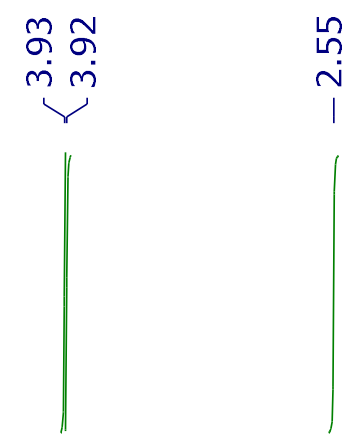

${ }^{1} \mathrm{H}$ NMR $\left(500 \mathrm{MHz}, \mathrm{CDCl}_{3}\right)$
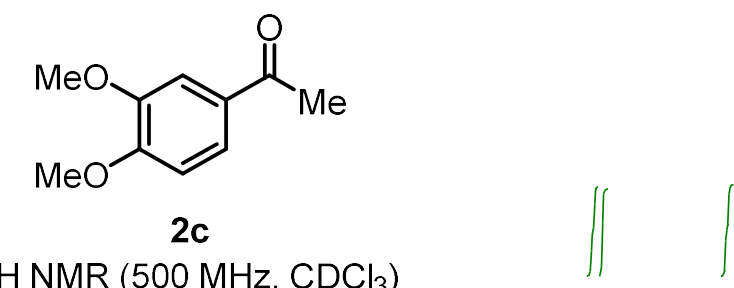

$$
\underset{1}{\stackrel{n}{n}}
$$

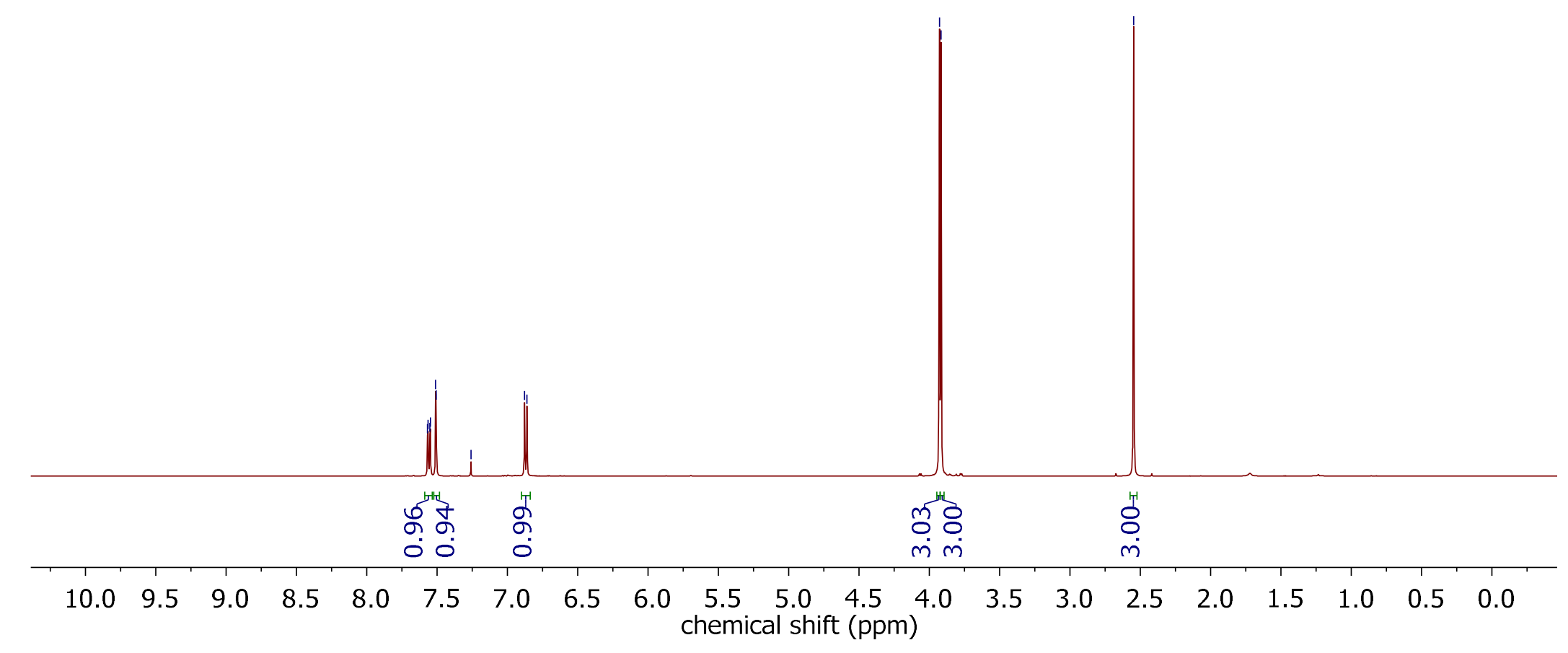




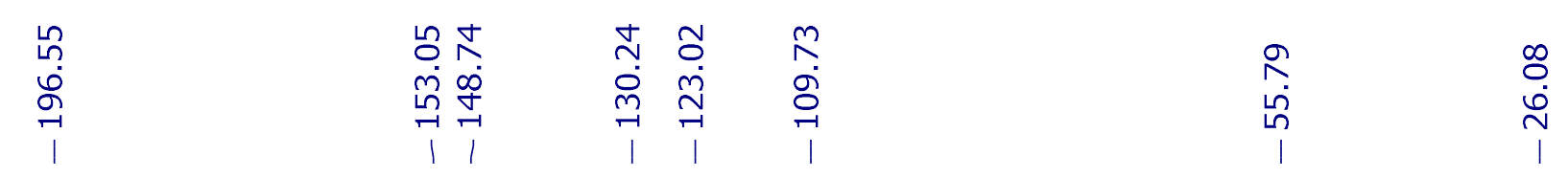

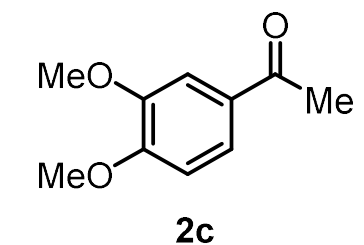

${ }^{13} \mathrm{C} \mathrm{NMR}\left(126 \mathrm{MHz}, \mathrm{CDCl}_{3}\right)$

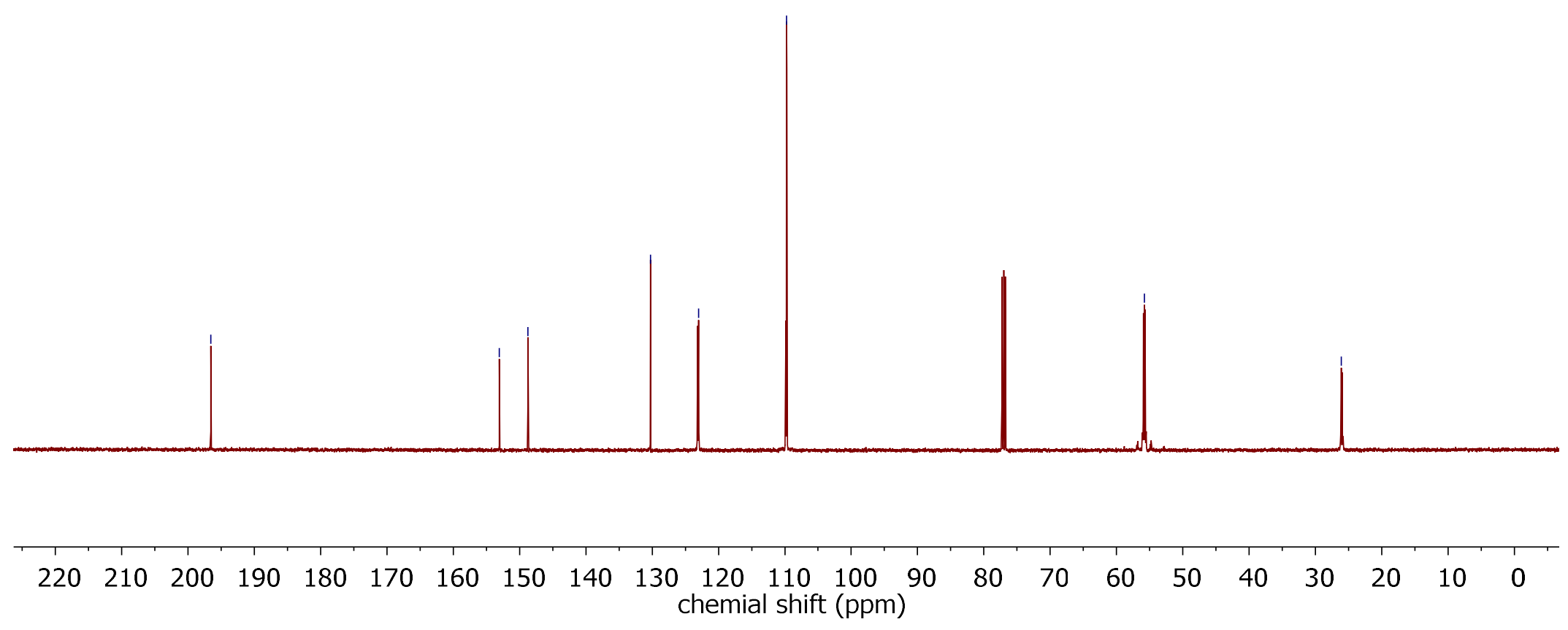




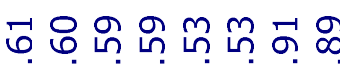

NヘNヘN

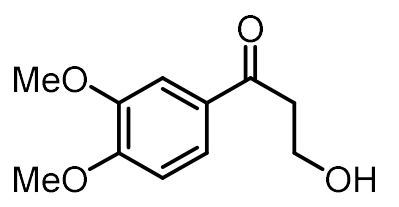

2d

${ }^{1} \mathrm{H} \mathrm{NMR}\left(500 \mathrm{MHz}, \mathrm{CDCl}_{3}\right)$

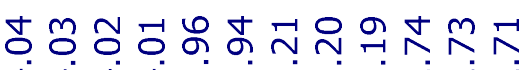

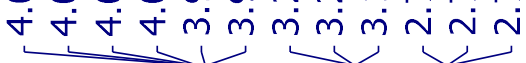

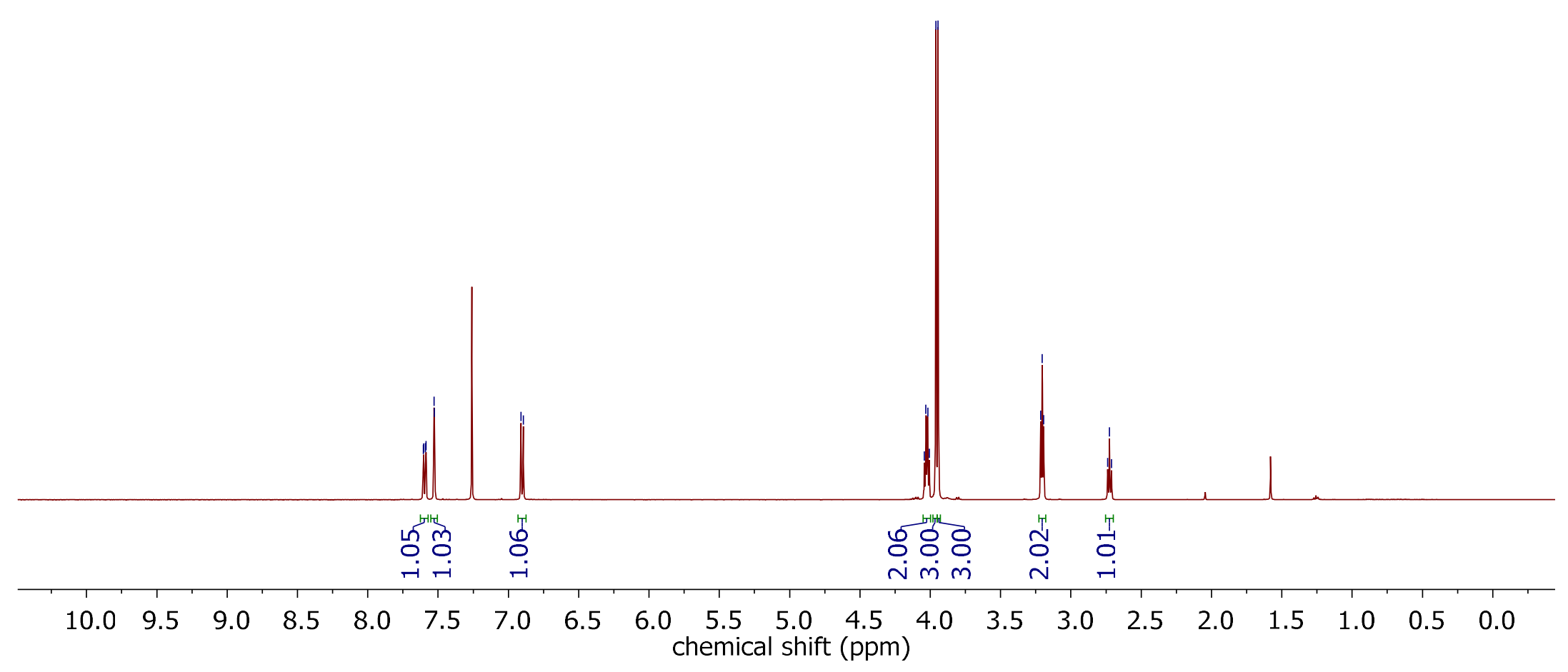




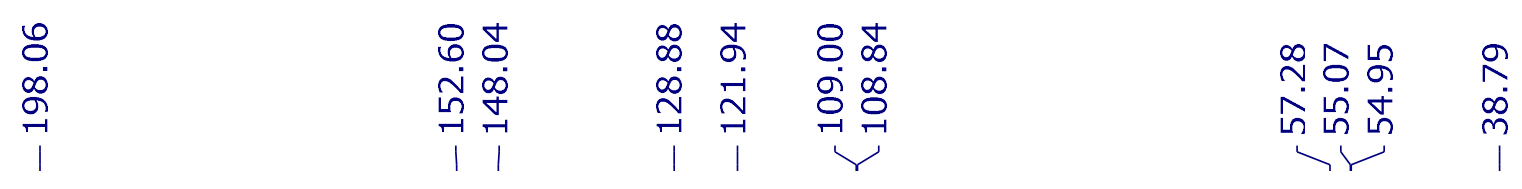

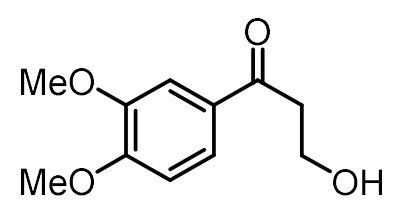

2d

$\left.{ }^{13} \mathrm{C} \mathrm{NMR} \mathrm{(126} \mathrm{MHz,} \mathrm{CDCl}_{3}\right)$

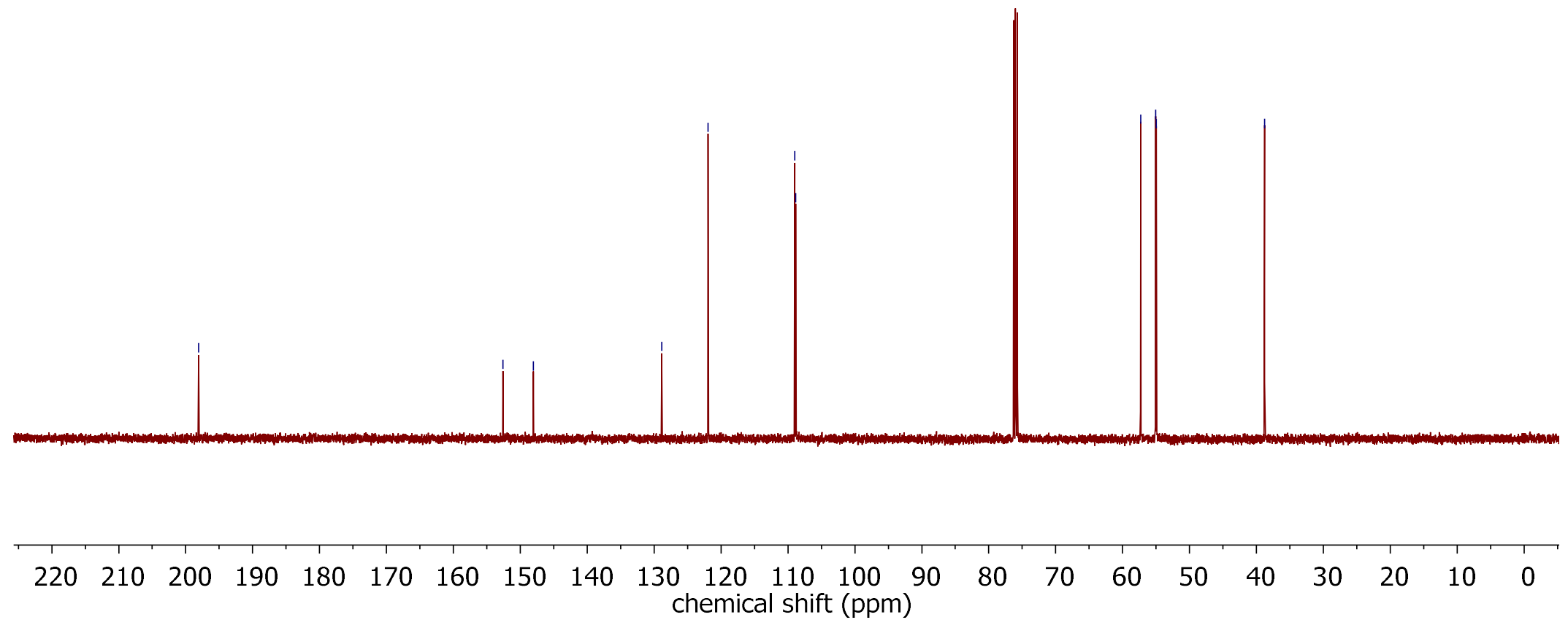




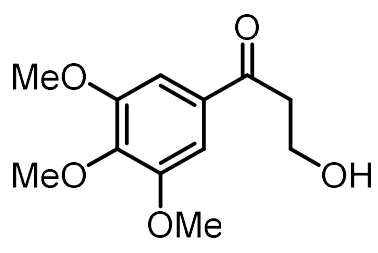

$2 e$

${ }^{1} \mathrm{H} \mathrm{NMR}\left(500 \mathrm{MHz}, \mathrm{CDCl}_{3}\right)$

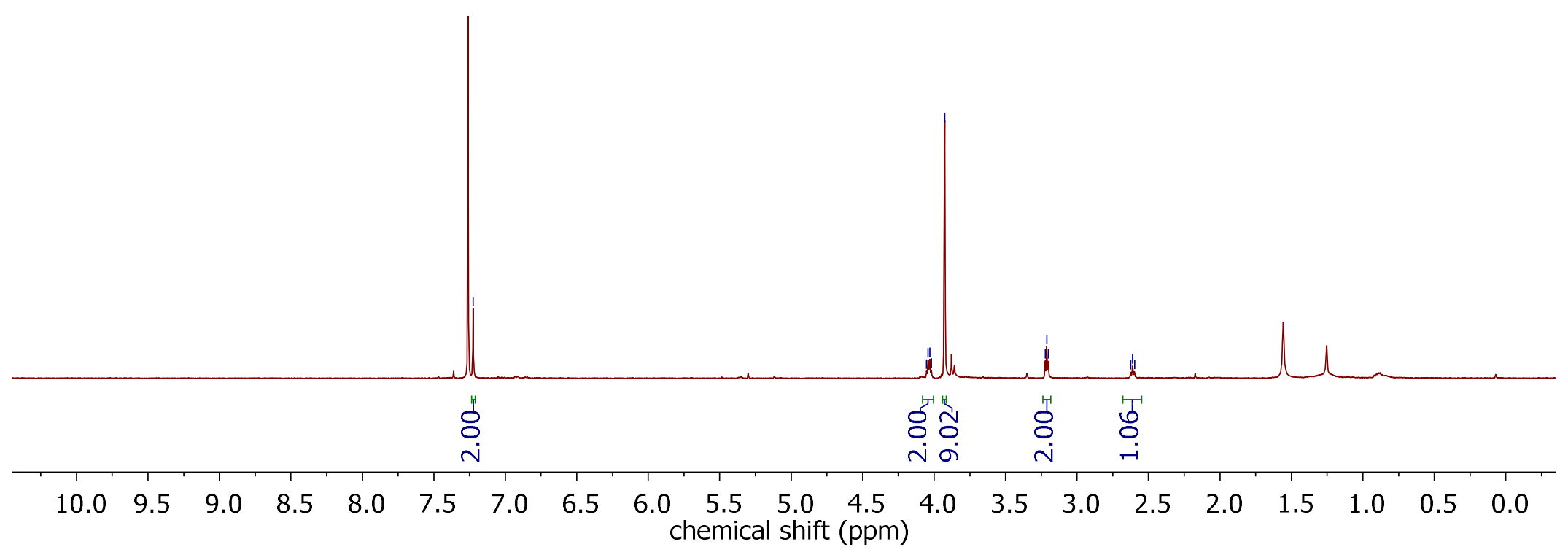



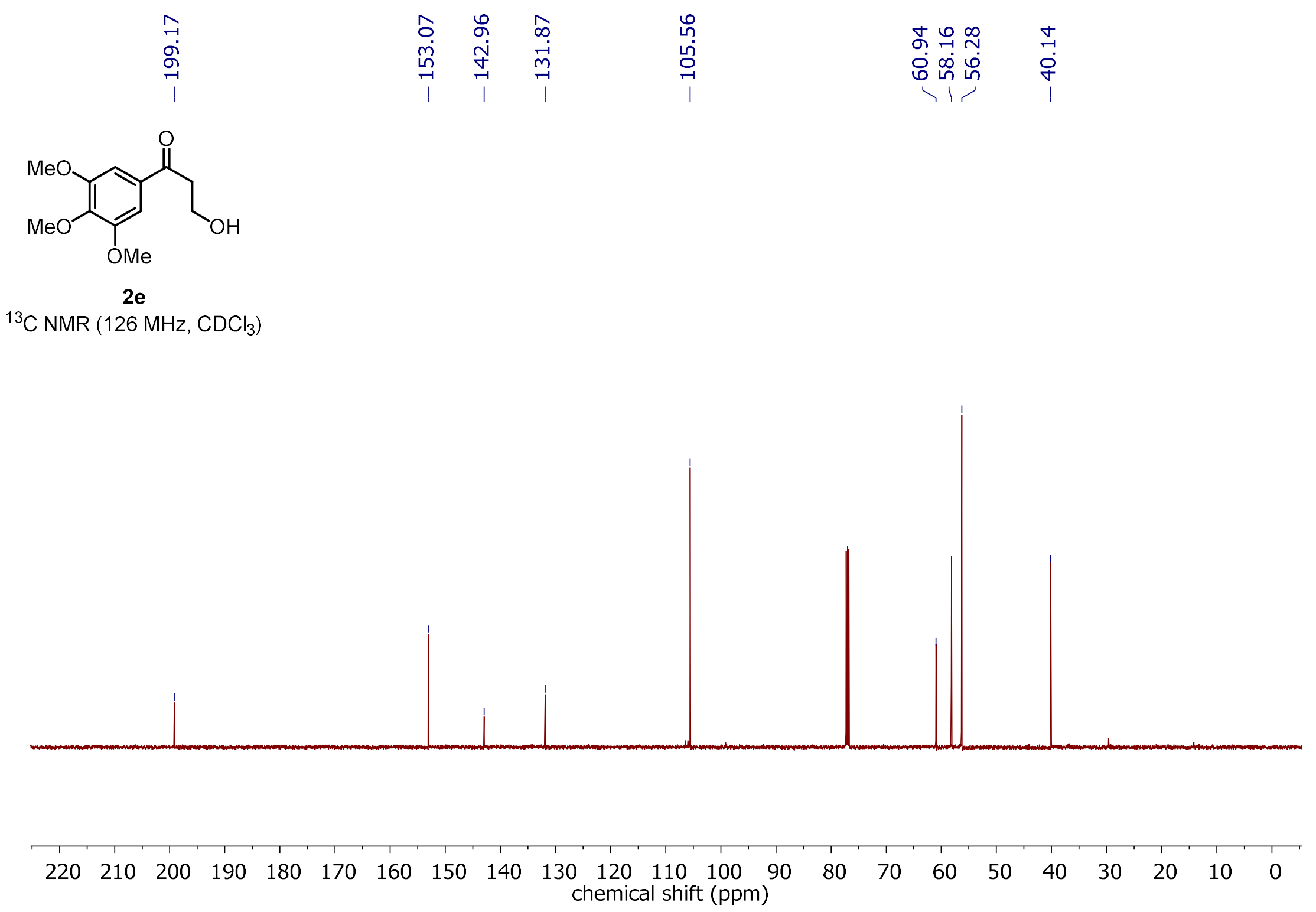


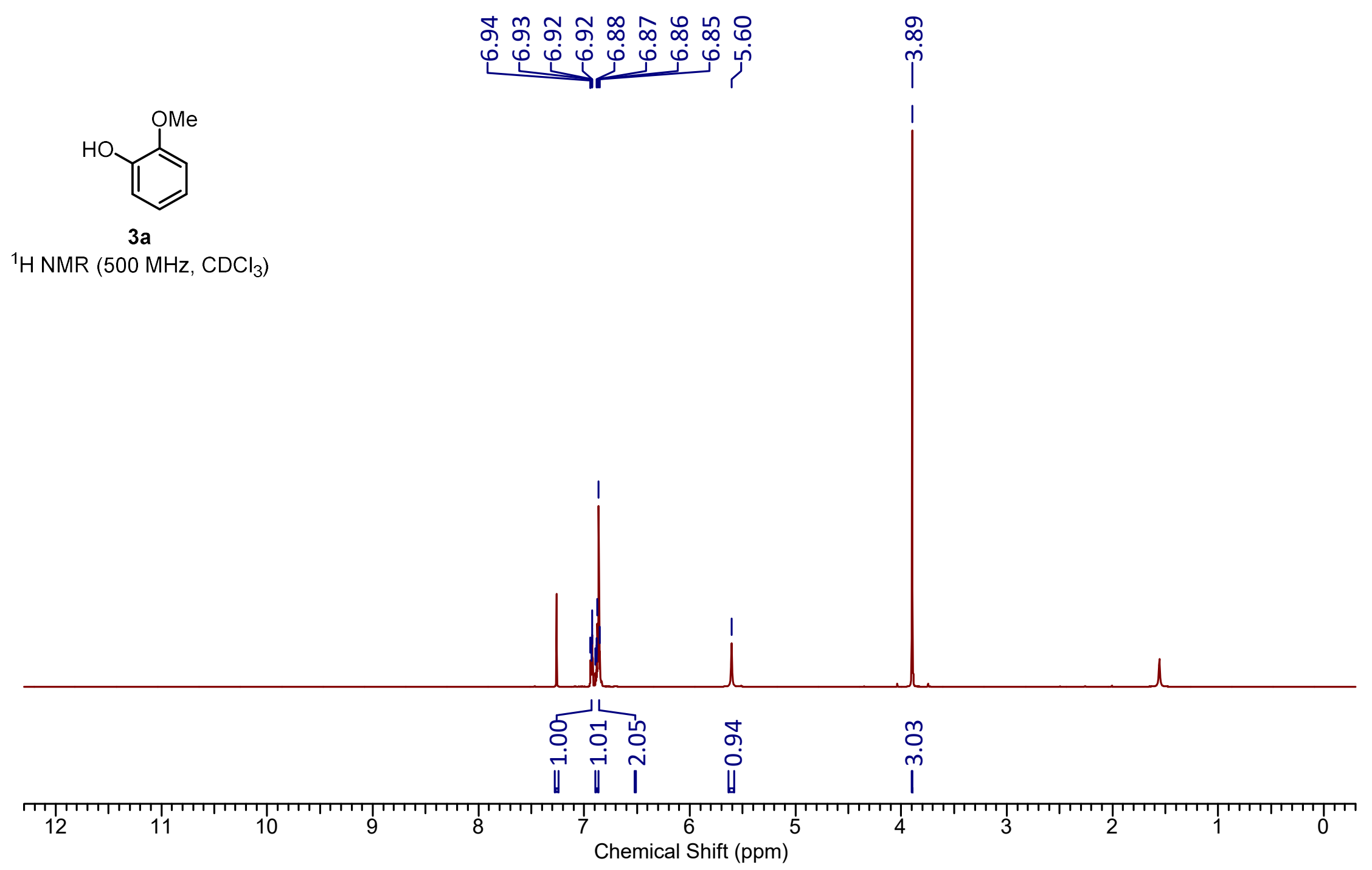




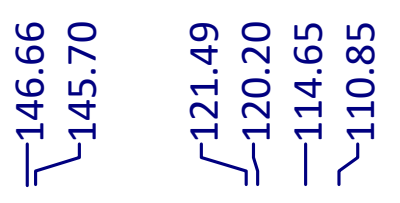

ڤึ

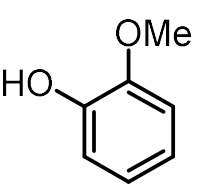

$3 a$

${ }^{13} \mathrm{C} \mathrm{NMR}\left(126 \mathrm{MHz}, \mathrm{CDCl}_{3}\right)$

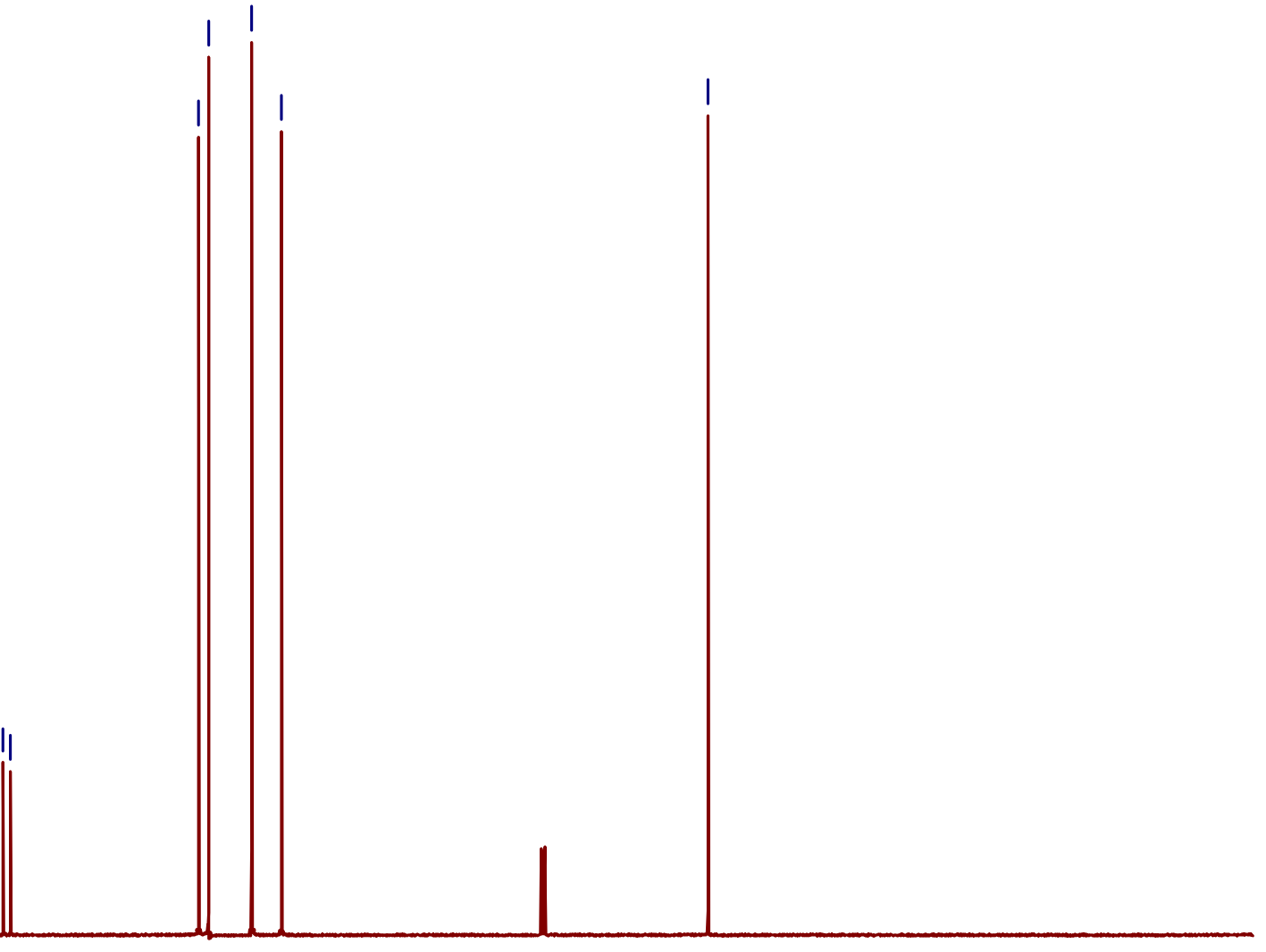

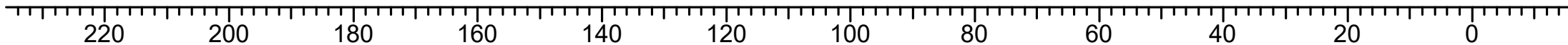
Chemical Shift (ppm) 


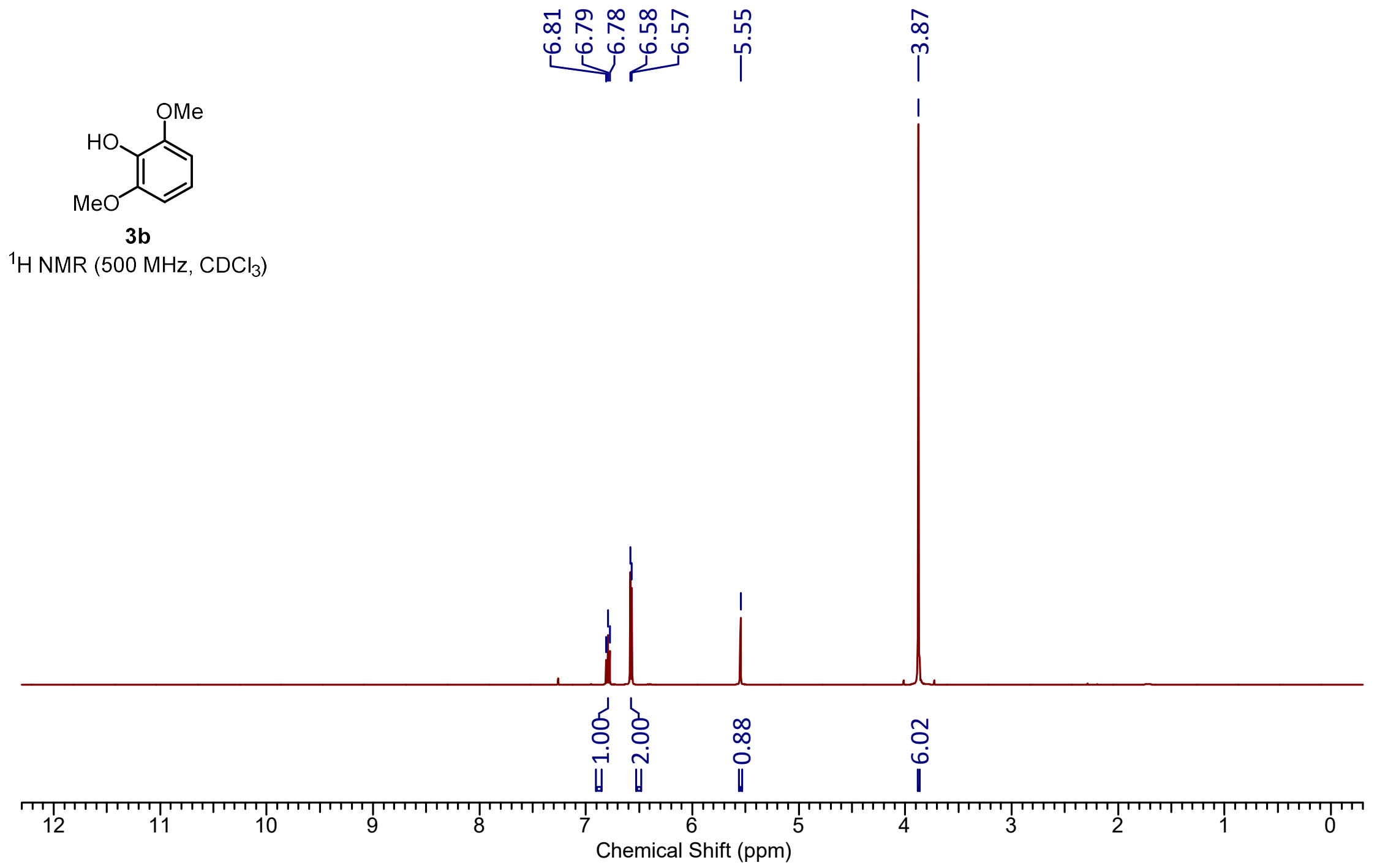



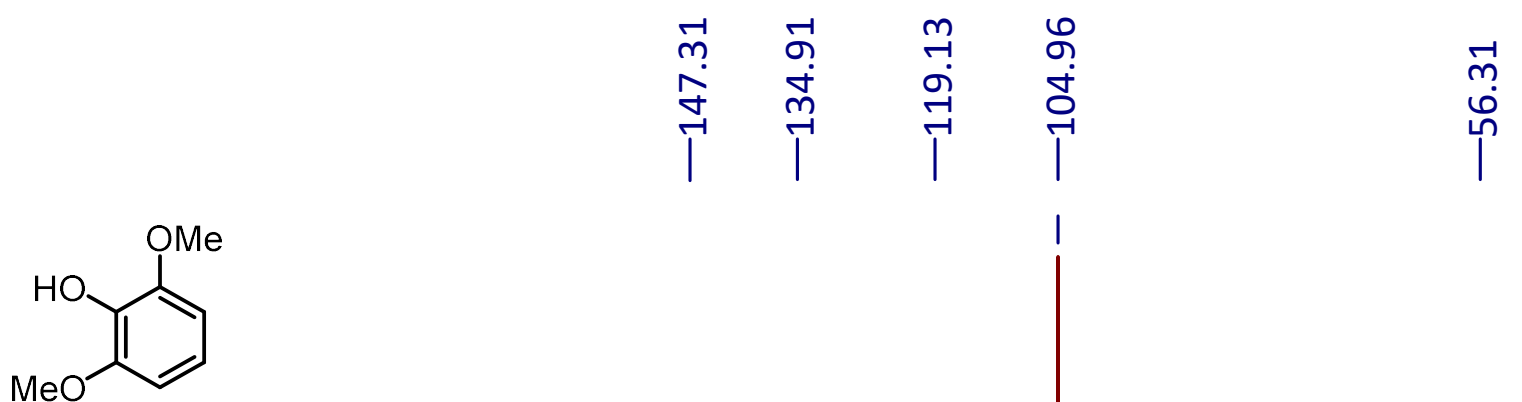

$3 b$

${ }^{13} \mathrm{C}$ NMR $\left(126 \mathrm{MHz}, \mathrm{CDCl}_{3}\right)$

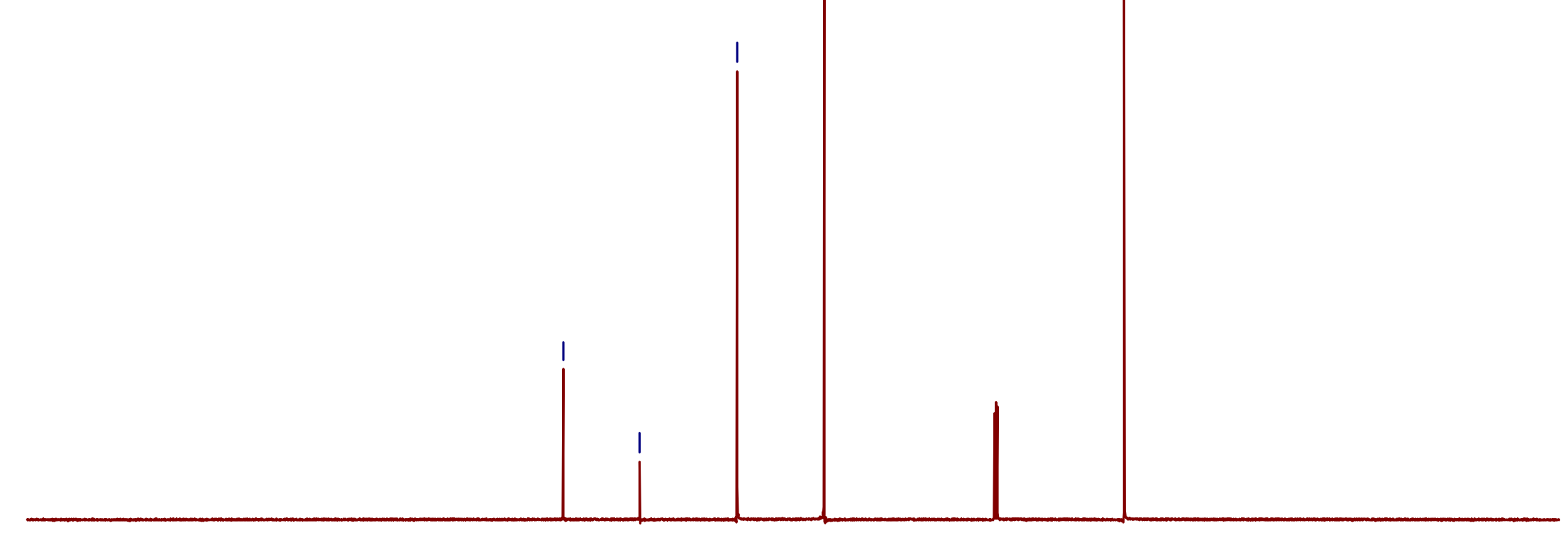

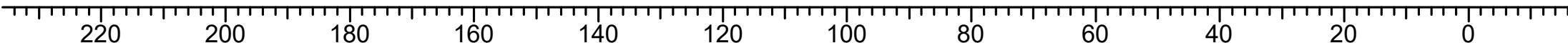




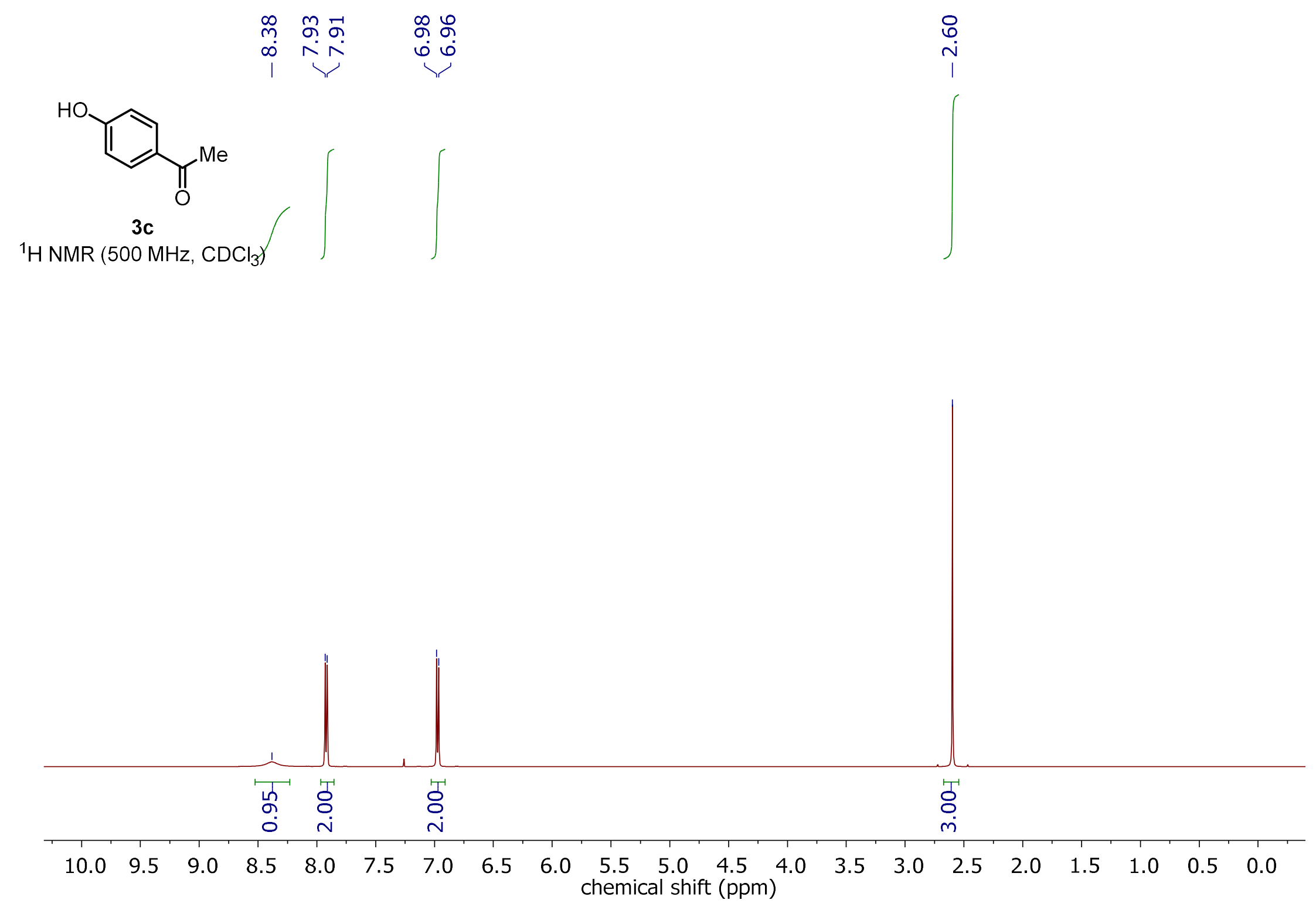



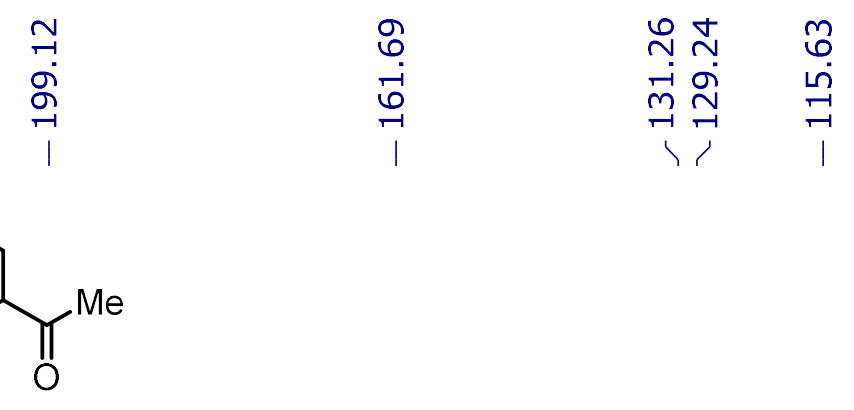

$\stackrel{m}{m}$

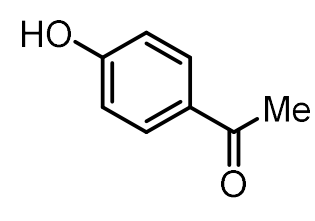

${ }^{13} \mathrm{C}$ NMR (126 MHz, CDCl 3 )
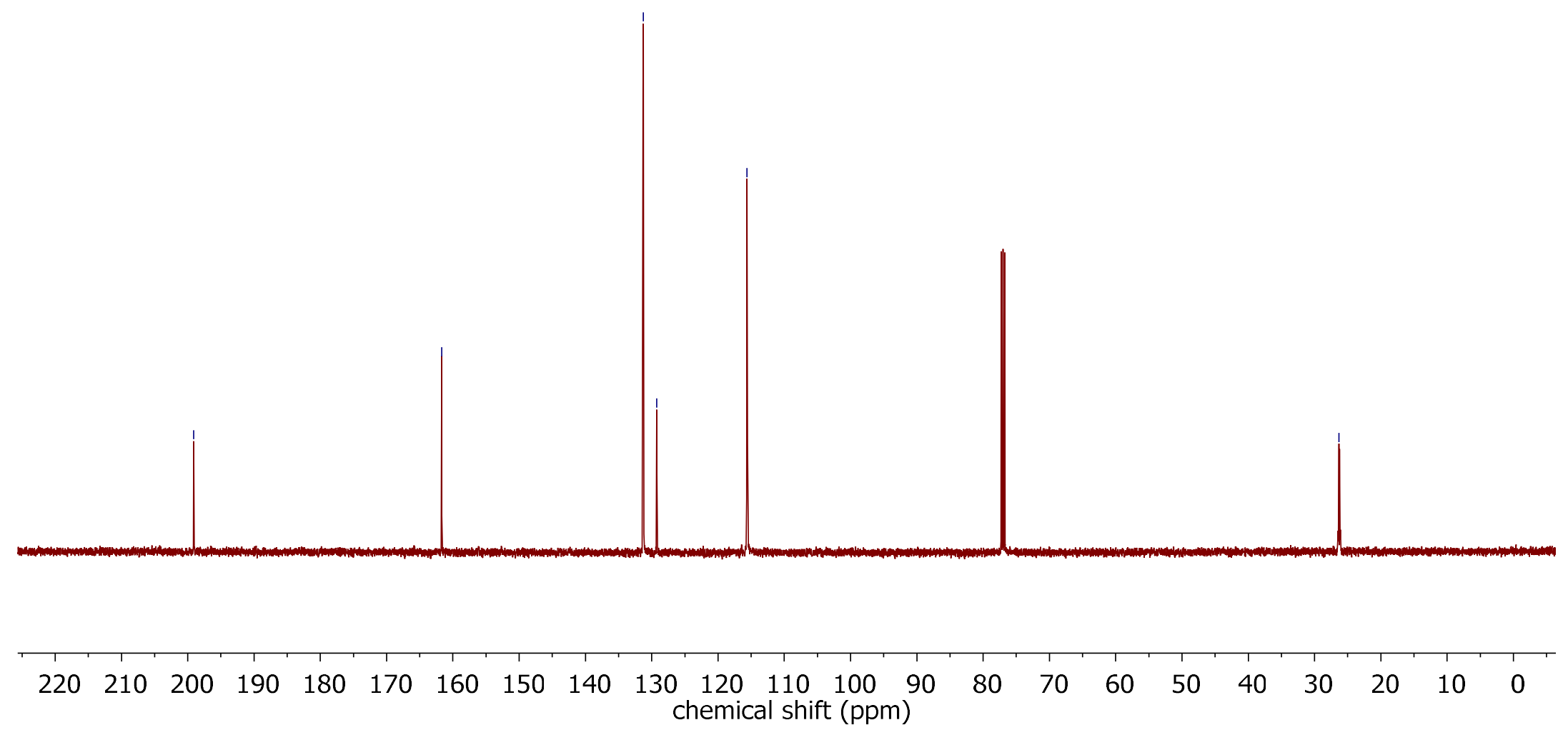